\title{
INTEGRABILITY IN QCD AND BEYOND
}

\author{
A. V. BELITSKY \\ Department of Physics and Astronomy, Arizona State University \\ Tempe, AZ 85287-1504, USA \\ and \\ Department of Physics, University of Maryland at College Park \\ College Park, MD 20742-4111, USA \\ V. M. BRAUN \\ Institut für Theoretische Physik, Universität Regensburg \\ D-93040 Regensburg, Germany \\ A. S. GORSKY \\ Institute of Theoretical and Experimental Physics \\ B. Cheremushkinskaya 25, Moscow, 117259 Russia \\ G. P. KORCHEMSKY \\ Laboratoire de Physique Théorique, Université Paris Sud \\ 91405 Orsay Cédex, France
}

\begin{abstract}
Yang-Mills theories in four space-time dimensions possess a hidden symmetry which does not exhibit itself as a symmetry of classical Lagrangians, but is only revealed on the quantum level. It turns out that the effective Yang-Mills dynamics in several important limits is described by completely integrable systems that prove to be related to the celebrated Heisenberg spin chain and its generalizations. In this review, we explain the general phenomenon of complete integrability and its realization in several different situations. As a prime example, we consider in some detail the scale dependence of composite (Wilson) operators in QCD and super-Yang-Mills (SYM) theories. Highenergy (Regge) behavior of scattering amplitudes in QCD is also discussed and provides one with another realization of the same phenomenon that differs, however, from the first example in essential details. As a third example, we address the low-energy effective action in a $\mathcal{N}=2 \mathrm{SYM}$ theory which, contrary to the previous two cases, corresponds to a classical integrable model. Finally, we include a short overview of recent attempts to use gauge/string duality in order to relate integrability of Yang-Mills dynamics with the hidden symmetry of a string theory on a curved background.
\end{abstract}

\footnotetext{
* Unité mixte de recherche du CNRS (UMR 8627).
} 
Table of Contents

1 Introduction 266

2 Light-cone dominated processes in QCD 274

2.1 Conformal svmmetrv and the collinear subgroup . . . . . . 276

2.2 Hamiltonian approach to operator renormalization . . . . . 279

2.3 Complete integrabilitv and non-compact Heisenberg magnets 285

2.4 Semiclassical expansion . . . . . . . . . . . . . . . . . . . 289

2.5 Breakdown of integrability . . . . . . . . . . . . . . . . . . . . . . . . . . . . . . . . . . . . . . .

2.6 Open spin chains . . . . . . . . . . . . . . . . . . 296

2.7 Quasipartonic and non-quasipartonic operators . . . . . . . 297

2.8 Conformal svmmetrv breaking . . . . . . . . . . . . . . . 300

3 Dilatation operators in supersymmetric gauge theories 301

3.1 SYM theories in light-cone superspace . . . . . . . . . . . . 302

3.2 Superconformal svmmetry on the light-cone . . . . . . . . . 305

3.3 One-loop dilatation operaton . . . . . . . . . . . . 308

3.4 Dilatation operator as a $S L(2 \mid \mathcal{N})$ Heisenberg spin chain . . . 311

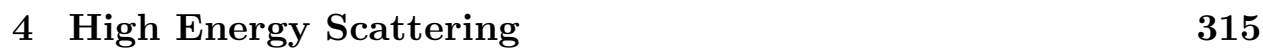

4.1 The BFKL Hamiltonian . . . . . . . . . . . . . . . . . . 316

4.2 Multireggeon compound states . . . . . . . . . . . 320

5 Spin chains and $\mathcal{N}=2$ super Yang-Mill theories $\quad 327$

6 Gauge/string correspondence 334

6.1 Derivation of the string in the thermodvnamical limit . . . . 337

6.2 Semiclassical string motion and integrable models . . . . . . . 338

6.3 Open string picture for anomalous dimensions . . . . . . . . . 342

7 Conclusion 343

\begin{tabular}{ll}
\hline References & 345
\end{tabular} 
We had the privilege and pleasure of knowing Ian Kogan as a close friend. In our informal chats it was clear that, whatever he was doing, there always was a single central problem that kept his mind constantly busy - the problem of confinement. All other projects he pursued - ranging from the Quantum Hall Effect to strings and quantum gravity - he considered from this general perspective. He viewed the issue of integrability as a unifying concept which could potentially explain many unsolved problems of strongly coupled theories and was very enthusiastic about this new development concerning four-dimensional gauge theories. If it were not for his untimely death, he would definitely have had a profound impact on the whole field with his usual passion for new concepts and trends.

\section{Introduction}

Understanding the dynamics of gauge theories at strong coupling is one of the outstanding problems in quantum field theory. It has been anticipated, since the classic work by Wilson [1], that the Yang-Mills dynamics at strong coupling can be reformulated as an effective theory of Faraday lines (gauge field flux) which, in its turn, can be reinterpreted as a string-like theory. A natural framework for discussing the stringy representation is offered by the multicolor limit. In this limit, one can express observables as a sum over surfaces (strong coupling expansion à la Wilson) but explicit realization of this program in gauge theories is still lacking due to absence of an operational gauge/string correspondence. It is expected that in the multicolor limit, the path integral over all gauge field configurations will be dominated by a saddle point - the so-called "master field". Polyakov has suggested [2] that the master field can be found as a solution to a two-dimensional sigma model defined on a nontrivial background (Lobachevski space-time). He also demonstrated that such models possess an infinite set of conserved currents and, therefore, can be solved exactly. It has long been speculated that similar nontrivial algebraic structures and, in particular, integrability can be revealed in four-dimensional quantum gauge theories as well. In the last few years several plausible indications that this actually occurs have appeared and several intriguing connections have been established between quantum gauge field theories and integrable lattice spin models. Recent progress has been in several directions, which we describe here.

At first glance, the connection between four-dimensional gauge theories and integrable systems may seem surprising. Indeed, in the latter case one is dealing with quantum-mechanical systems, which have a finite number of degrees of freedom and the same number of conserved charges. For such 
systems the degrees of the freedom are just phase-space variables involved in the Hamiltonian and the "evolution time" has a literal physical meaning. In contrast, Yang-Mills theories in four dimensions are complex systems with infinitely many degrees of freedom which are not integrable per se. Complete integrability emerges as a unique feature of effective Yang-Mills dynamics in various limits. The relevant degrees of freedom, Hamiltonians and "evolution times" within the Yang-Mills theory are different in different limits and their identification is not a priori obvious. In this work we will elaborate on three examples which illustrate the general phenomenon of hidden integrability. They are

- Scale dependence of composite (Wilson) operators in QCD and super-Yang-Mills (SYM) theory

- High-energy (Regge) behavior of scattering amplitudes in QCD

- Low-energy effective action in $\mathcal{N}=2$ SYM theory

In all three cases, the dynamics described by an integrable model corresponds to the scale dependence of corresponding observables with the "evolution time" being identified as the logarithm of the relevant energy scale. In the first two cases one is dealing with quantum integrable models, while the $\mathcal{N}=2$ low-energy action corresponds to a classical model. The integrable systems that emerge in this context turn out to be related to the celebrated Heisenberg spin magnet and its offspring. This model was introduced by Heisenberg in 1926 and describes a one-dimensional chain of atoms with an exchange interaction,

$$
\mathbb{H}_{s=1 / 2}=-\sum_{n=1}^{L}\left(\mathbf{S}_{n} \cdot \mathbf{S}_{n+1}-\frac{1}{4}\right)
$$

where $\mathbf{S}_{n}=\left(S_{n}^{x}, S_{n}^{y}, S_{n}^{z}\right)$ is the spin-1/2 operator of the $n$-th atom in the chain of length $L$ and periodic boundary conditions are implied: $\mathbf{S}_{L+1}=\mathbf{S}_{1}$. The model (1.1) is completely integrable and its eigenspectrum was found in 1931 by Bethe by an innovative method which is widely known nowadays as the Bethe Ansatz. Much later, it was understood [3,4] that the original Heisenberg model (1.1) can be generalized to arbitrary spins while preserving complete integrability. The Hamiltonian of a completely integrable lattice model describing a chain of interacting spin $-s$ operators was found to be [4]

$$
\mathbb{H}_{s}=\sum_{n=1}^{L} H\left(J_{n, n+1}\right), \quad J_{n, n+1}\left(J_{n, n+1}+1\right)=\left(\mathbf{S}_{n}+\mathbf{S}_{n+1}\right)^{2}
$$


Here the operator $J_{n, n+1}$ is related to the sum of two spins in the neighboring sites, $\mathbf{S}_{n}^{2}=s(s+1)$, and $H(x)$ is the following harmonic sum

$$
H(x)=\sum_{l=x}^{2 s-1} \frac{1}{l+1}=\psi(2 s+1)-\psi(x+1),
$$

where $\psi(x)=d \ln \Gamma(x) / d x$ is the Euler $\psi$-function. For $s=1 / 2$, the twoparticle spin takes the values $J_{n, n+1}=0$ and $J_{n, n+1}=1$. In that case, $H(0)=1$ and $H(1)=0$ so that the two-particle Hamiltonian $H\left(J_{n, n+1}\right)$ is given by a projector onto $J_{n, n+1}=0$ subspace, $H\left(J_{n, n+1}\right)=\frac{1}{4}-\mathbf{S}_{n} \cdot \mathbf{S}_{n+1}$, in agreement with (1.1).

Approximately at the same time as the model (1.2) was formulated, QCD calculations of the anomalous dimensions of twist-two Wilson operators [5] and high-energy asymptotics of scattering amplitudes [6] led to expressions involving the very same combination $[\psi(J)-\psi(1)]$ with $J$ being the Lorentz and conformal $S L(2)$ spin respectively. In both situations, the appearance of the $\psi$-functions is a generic feature related to the existence of massless vector fields (gluons). For almost two decades, this similarity remained unnoticed, mainly because of a lack of interaction between the two communities. Matching the QCD expressions with (1.2), one discovers the hidden integrability properties of gauge theories [7-10]. Approximately at the same time hidden integrability has been found in the $\mathcal{N}=2$ supersymmetric Yang-Mills theory [11]. In the three cases mentioned above, the identification goes as follows.

In the so-called Bjorken kinematic limit for "hard" scattering processes, involving a large momentum transfer to a hadronic system, the shortdistance perturbative QCD dynamics can often be separated (factorized) from the large-distance interactions and described through a set of gaugeinvariant, local composite operators built from fundamental fields and covariant derivatives. These (Wilson) operators mix under renormalization and their scale dependence is governed by the renormalization group (RG) or Callan-Symanzik equation

$$
\mu \frac{d}{d \mu} \mathcal{O}_{N}(x)=\sum_{K} \gamma_{N K}(g) \mathcal{O}_{K}(x),
$$

where $\gamma_{N K}$ is the mixing matrix given by a series in the running coupling constant $g=g\left(\mu^{2}\right)$. The size of the mixing matrix is constrained by the symmetries and depends on the operators under consideration. The matrix $\gamma_{N K}$ can be interpreted as a Hamiltonian acting in the space of operators that get mixed via the RG flow [12] with logarithm of the RG scale $\tau=\ln \mu$ 
playing the rôle of the "evolution time". In this way, the evolution equation (1.4) takes the form of a Schrödinger equation. It turns out that for a certain subclass of operators, and to one-loop accuracy, the corresponding Hamiltonian can be identified as that of the open and/or closed Heisenberg magnet with the spins being the generators of the $S L(2, \mathbb{R})$ group $[10,13,14]$. The number of sites in the spin chain is given by the number of fundamental fields involved in the composite operators and the value of the spin at each site is fixed by the $S L(2, \mathbb{R})$ representation to which the corresponding field belongs. It is different for quarks and gluons. The emergence of the $S L(2, \mathbb{R})$ group as a symmetry group of the spin chain is not accidental, since this group is just a reduction of the four-dimensional conformal group $S O(2,4)$ for field operators "living" on the light-cone $[15,16]$. Integrability allows one to apply the Bethe Ansatz to reconstruct the spectrum of the anomalous dimensions $[13,14,17]$. Work in this direction [18-22] has led to an almost complete understanding of the spectrum of anomalous dimensions of twistthree operators in QCD which are important for phenomenology. It has to be mentioned that, as a rule, QCD evolution equations only become integrable in the large $N_{c}$ limit. There is no chance that a quantum $S U\left(N_{c}\right)$ theory would turn out to be integrable for any $N_{c}$ because the phenomena it describes are too complicated - nuclear forces being one of these. Indeed, in QCD it was found that corrections that break integrability depend on the quantum numbers of the operators (states) and the structure of such corrections are such that they lead to creation of "mass gaps" in the spectrum of anomalous dimensions which can be interpreted as creation of bound states in the corresponding quantum mechanical model [18].

Although historically the phenomenon of integrability was first discovered in QCD for operators with maximum helicity, it is, in fact, a general hidden symmetry of all Yang-Mills theories, which is not manifest an the classical level and is enhanced in its supersymmetric extensions [23]. In a supersymmetric Yang-Mills theory, the mixing matrix in (1.4) gets modified due to the presence of additional fields. Their contribution preserves the QCDtype integrability and further augments it to an increasingly growing class of operators as one goes from pure Yang-Mills $(\mathcal{N}=0)$ to the maximallysupersymmetric $\mathcal{N}=4 \mathrm{SYM}$ theory [24]. In particular, in the $\mathcal{N}=4 \mathrm{SYM}$ theory, integrability holds in the sector of scalar operators [25, 26]. This theory involves three complex scalars and the one-loop mixing matrix for the scalar operators of the type $\operatorname{tr}\left\{\Phi_{1}^{J_{1}}(0) \Phi_{2}^{J_{2}}(0) \Phi_{3}^{J_{3}}(0)\right\}$ can be identified as the Heisenberg $S O(6)$ spin chain with $J_{1}+J_{2}+J_{3}$ sites. The $S O(6)$ group is nothing but the $R$-symmetry group of the model with six real scalars 
belonging to its fundamental representation. As a natural generalization of these two integrable structures, discovered independently, it was found that the one-loop renormalization of operators involving gauge, fermions and scalar fields in $\mathcal{N}=4 \mathrm{SYM}$ is described by a spin chain with $S U(2,2 \mid 4)$ group representations on each site [23].

As a second example, we consider the high-energy (Regge) asymptotics of scattering amplitudes. The scattering amplitude $\mathcal{A}(s, t)$ in QCD in the large- $N_{c}$ limit (here $s, t$ are Mandelstam variables) can be written in the Regge limit $s \gg-t$ as

$$
\mathcal{A}(s, t)=\sum_{N=2}^{\infty} \mathcal{A}_{N}(s, t), \quad \mathcal{A}_{N}(s, t) \sim s^{\lambda E_{N}},
$$

where $\lambda=g^{2} N_{c}$ is the 't Hooft coupling constant and the positive integer $N$ can be thought of as the number of (reggeized) gluons exchanged in the $t$-channel. The partial amplitudes $\mathcal{A}_{N}(s, t)$ have a power-like energy dependence which is governed by the numbers $E_{N}$. It turns out that the $E_{N}$ coincide with the ground state energies of a completely integrable spin chain model [7-9]. The length of the spin chain $N$ equals the number of the reggeized gluons involved, the "evolution time" is given by the total rapidity $\tau=\ln s$ and the relevant degrees of freedom are the two-dimensional transverse coordinates of the reggeized gluons in the scattering plane. This model can be identified as a homogeneous Heisenberg magnet (1.2) with the spin operators $\mathbf{S}_{n}$ being the generators of a (infinite-dimensional) unitary representation of the $S L(2, \mathbb{C})$ group. This model can be solved by the Bethe Ansatz and this allows one to calculate the spectrum of $E_{N}[27,28]$.

We should stress that integrability of reggeon interactions is not specific for QCD. Since the dominant contribution to the scattering amplitudes comes from $t$-channel exchange of particles with maximal spins (gluons), it is not altered (in the leading logarithmic approximation) by the presence of additional fields in SYM theories. In other words, the phenomenon is tied to the gauge sector of a SYM theory.

Finally, the third example of integrability is provided by the SeibergWitten solution for the low-energy effective action of the $\mathcal{N}=2$ SYM theory [29]. This solution is described by a Riemann surface bundled over the space of order parameters characterizing the vacuum state of the $\mathcal{N}=2$ SYM theory. This surface fixes both the low-energy effective action depending on the massless low-energy modes $u_{k}=\left\langle\operatorname{tr} \phi^{k}\right\rangle$ and the spectrum of stable BPS states in the theory. The Seiberg-Witten solution admits an elegant interpretation in the context of classical integrable models $[11,30,31]$. The 
particular pattern of the integrable model depends on the matter content in the $\mathcal{N}=2$ theory, involving examples of Toda and Calogero systems, as well as different types of spin chains. The number of degrees of freedom in the underlying integrable system is equal to $N_{c}-1$ for gauge group $S U\left(N_{c}\right)$. As in previous examples, the crucial point is the identification of the "time" variable. In the present case, the time is related to the fundamental scale of the $\mathcal{N}=2 \mathrm{SYM}$ theory, $\tau=\ln \Lambda_{\mathrm{QCD}}$, which arises through the dimensionaltransmutation phenomenon. The gauge-invariant order parameters $u_{k}$ turn out to be higher Hamiltonians of the integrable model, while the BPS spectrum coincides with the action variables.

The phenomenon of integrability has two different aspects. From the point of view of phenomenology, it allows one to apply the powerful Quantum Inverse Scattering Method [32] to solve problems which cannot be treated by standard techniques. Recent applications include the calculation of the spectrum of the multi-reggeon compound states responsible for the powerlaw rise of the scattering amplitude as a function of the energy and the exact solution of the evolution equations for multi-particle distribution amplitudes in QCD. On the theory side, integrability leaves a lot of questions to be answered. Currently, it has the status of an "experimental observation" and its origin within the Yang-Mills dynamics remains unclear. It is natural to ask whether integrability survives at higher loops, what the meaning of the higher integrals of motion on the Yang-Mills side is and what the impact of non-perturbative effects is. It is unlikely that the answers can be found in the framework of perturbation theory. The string/gauge duality provides an alternative approach. It offers a unifying picture for the gauge theory both at weak and strong coupling regimes and allows one to relate integrability of the Yang-Mills theory with the hidden symmetry of a (non)critical string theory propagating in the curved background [33-36]. ${ }^{\text {a }}$

Presently the gauge/string duality is rather firmly established only for the maximally-supersymmetric gauge theory [38]. According to this conjecture, at large $N_{c}, \mathcal{N}=4 \mathrm{SYM}$ is equivalent to a string theory with $A d S_{5} \times S_{5}$ target space and the flux of the four-form field inducing the number of colors. It is assumed that the radii of $A d S_{5}$ and $S_{5}$ coincide and the tension of the string is proportional to $\left(g^{2} N_{c}\right)^{1 / 2}$. At strong coupling, the tension is large and the string can be treated semi-classically. On the other hand, the weak coupling regime in the field theory is mapped into the quantum regime in the string sigma model. Unfortunately there exists no satisfactory quantum treatment

a Similar nonlocal Yangian type symmetries were previously discussed in the context of high-energy asymptotics in QCD in Ref. [37]. 
of the string in the $A d S_{5} \times S_{5}$ background. Therefore, it is necessary to select proper objects both on the gauge theory and string sides to make their comparison explicit.

The anomalous dimensions of the gauge theory operators appear to be very convenient objects for such a comparison. The dilatation operator in the gauge theory can be identified with the Hamiltonian on the stringy side so that calculation of the anomalous dimension of the operator is equivalent to the calculation of the energy of the corresponding string configuration $[39,40]$. Lacking the whole quantum spectrum of the string, the analysis can be performed only in the special, exactly solvable subsectors of the string theory, or for selected operators in the gauge theory which can be treated semi-classically on the string side. The exactly-solvable example deals with a string moving in the pp-background, which corresponds to the so-called BMN operators [39], involving scalars, in the $\mathcal{N}=4$ SYM theory. This is the only example of a gauge theory operator whose anomalous dimensions are known at all values of the gauge coupling constant. A more general class of operators with large quantum number with respect to the $R$-symmetry group allow a semi-classical stringy treatment and the comparison of the stringy calculation with the perturbative expansion in Yang-Mills theory.

The integrability arises along these lines in two different ways. In the strong coupling regime, we treat the string classically and a finitedimensional integrable system of the Neumann type emerges if one assumes a simple ansatz for the string motion [41]. On the other hand, one can start with the one-loop calculation at weak coupling and consider the thermodynamical limit of the spin chain. In this limit, in the coherent state basis, the string sigma model becomes manifest, with each field incorporated into the composite operator playing the rôle of a single string bit [42].

The mapping of the integrable system to the stringy picture behind the low-energy effective actions is slightly different. In this case, the spectral curve of the integrable system is part of the background since the M5 brane providing the world-volume for the four-dimensional theory under consideration is wrapped around it [43]. The states of the string wrapped around the spectral curve amount to the spectrum of BPS states in the gauge theory which is a counterpart of the gauge/string correspondence in this case. The first step towards the derivation of the stringy picture for the Regge case based on the semiclassical limit of the $S L(2, \mathbb{C})$ spin chains was done in [44].

The presentation is organized as follows. In Sect. 2, we explain the phenomenon of hidden integrability for the scale dependence of local operators, alias the renormalization group $(\mathrm{RG})$ dilatation operator. We consider 
three-quark operators in some detail, and using this example illustrate the main steps that lead to the spin-chain interpretation. This includes the treatment of RG equations as a Hamiltonian problem, finding an explicitly $S L(2)$-covariant representation and identification of the conserved charges. The main approaches used in the quantum integrability framework are described, including the method of Separated Variables, the method of the Baxter $Q$-operator and semiclassical solution to the Baxter equation in terms of Riemann surfaces. We will demonstrate that integrability in QCD is restricted to operators built of fundamental fields with maximal helicity (and covariant derivatives), whereas the "interaction" between quarks with opposite helicity breaks integrability and leads to creation of mass gaps in the spectrum. We also give a short summary of the applications to quarkantiquark-gluon operators, in which case open spin chains arise.

In Sect. 3, we consider the extension of these results to the case of SYM theories using the non-covariant light-cone superspace formalism due to Mandelstam [45] and Brink et al [46]. We give a short review of this formalism and present the results of the one-loop calculation of the dilatation operator in the $\mathcal{N}=2$ and $\mathcal{N}=1$ SUSY theories. We explain how the super spin chains emerge in the dilatation operator, which we map to a $S L(2 \mid \mathcal{N})$ Heisenberg (super)spin chain.

Section 4 is devoted to the high-energy asymptotics of scattering amplitudes. We explain both the differences and the similarities of this problem with the case of local-operator renormalization and give a short summary of the existing results. In this way, we identify the compound states of reggeized gluons in multicolor QCD as ground states of the quantum spin chain with $S L(2, \mathbb{C})$ group. We present the exact solution to the eigenproblem for this integrable model based on the method of the Baxter $Q$-operator and discuss the properties of the energy spectrum within the semiclassical approach.

Section 5 contains a discussion of the low-energy effective actions in SYM while in Sect. 6 we give an overview of the relation between the spectrum of the anomalous dimensions of composite operators in gauge theory and energies of the string in some background within the string/gauge duality. In particular, the operators with large quantum numbers will be described semi-classically on the stringy side. The mapping between integrable spin chains and strings will be briefly outlined. The final Sect. 7 contains a summary and concluding remarks. 


\section{Light-cone dominated processes in QCD}

For a practitioner, QCD as a theory of strong interactions has been mainly of use in scattering processes at high energies. In this case, world lines of quarks and gluons participating in the scattering event are close to the light cone. It is therefore not surprising that the separation of transverse and longitudinal coordinates with respect to the scattering plane proves to be essential. Thus, before going into details let us introduce some notation.

Let $n$ and $\bar{n}$ be two independent light-like vectors

$$
n^{2}=\bar{n}^{2}=0, \quad n \cdot \bar{n}=1 .
$$

For an arbitrary four-vector, $A_{\mu}$ we define

$$
A_{+} \equiv A_{\mu} n^{\mu}, \quad A_{-} \equiv A_{\mu} \bar{n}^{\mu},
$$

and the metric tensor in the directions orthogonal to the light-cone

$$
g_{\mu \nu}^{\perp}=g_{\mu \nu}-n_{\mu} \bar{n}_{\nu}-n_{\nu} \bar{n}_{\mu} .
$$

We will also use the notation $A_{\perp}$ for a generic transverse projection and $A_{\perp}^{\mu}=\left(0, \boldsymbol{A}_{\perp}, 0\right)$ for a vector that only has transverse components. For example,

$$
x^{\mu}=x_{-} n^{\mu}+x_{+} \bar{n}^{\mu}+x_{\perp}^{\mu}, \quad x_{\perp}^{\mu} \equiv g_{\perp}^{\mu \nu} x_{\nu}
$$

and, therefore, $x^{2}=2 x_{+} x_{-}-\boldsymbol{x}_{\perp}^{2}$ with $\boldsymbol{x}_{\perp}^{2}=-x_{\perp} \cdot x_{\perp}$.

In the parton model, hadron states are described by a bunch of partons, all moving in the same light-like direction, say $\bar{n}_{\mu}$, and bound in a hadron wave function. If, in addition to high energy, the physical process of interest also involves a large momentum transfer, then in a rather generic situation the transverse structure of the parton system is not resolved by the interaction and appears to be irrelevant. For such processes, which we refer to as light-cone dominated, the nonperturbative hadron structure is encoded in suitable matrix elements of gauge-invariant nonlocal operators built of quark, antiquark and gluon fields located on the same light-ray (hence the name light-ray operators [47]) and connected by light-ray ordered gauge links (Wilson lines). For example,

$$
\bar{\psi}\left(z_{1} n\right) \not \mathrm{P} \exp \left\{i g \int_{z_{2}}^{z_{1}} d u A_{+}(u n)\right\} \psi\left(z_{2} n\right),
$$

where $\psi(x)$ is a quark field and $z_{i}$ 's are real numbers $z_{i} \equiv x_{i-}$. In what follows we will not show the gauge links in order not to complicate the 
formulae. However, they are always implied. ${ }^{\mathrm{b}}$

As always in a field theory, taking the asymptotic limit (here, by approaching the light-cone) induces extra divergences in addition to conventional ultraviolet ones. Both have to be renormalized. The nonlocal light-ray operator can be understood as the generating function of renormalized local operators, e.g.,

$$
\bar{\psi}(-z n) \not h \psi(z n)=\sum_{N} \frac{(2 z)^{N}}{N !} \bar{\psi}(0) \not h \stackrel{\leftrightarrow}{D}_{+}^{N} \psi(0),
$$

where $\stackrel{\leftrightarrow}{D}_{+}=n \cdot \vec{D}-n \cdot \overleftarrow{D}, D_{\mu}$ is the covariant derivative. Note that the contraction of covariant derivatives with the light-like vector picks up the contribution of the maximum Lorentz spin, and all arising local operators have the same twist $=$ dimension - spin. The operators in (2.5) can mix with similar operators containing total derivatives. However, the corresponding mixing matrix is triangular, so that the anomalous dimensions are not affected and can be calculated by taking the matrix elements over states with equal momenta, in which case operators with total derivatives do not contribute. For a given $N$, therefore, one is left with a single anomalous dimension $\gamma_{N}$, and the corresponding expression has been known since the pioneering work in [5]. By now it is known to three-loop accuracy. Taking into account the mixing with gluons makes $\gamma_{N}$ a $2 \times 2$ matrix, but does not add complications of principle.

Going over to operators built from three and more fields, the situation becomes considerably more involved. In the bulk of this section we will consider a particular example of the light-ray operators built out of three quark fields of definite helicity $\psi^{\uparrow(\downarrow)}=\frac{1}{2}\left(1 \pm \gamma_{5}\right) \psi$ at a light-like separation

$$
\begin{aligned}
& B_{\alpha \beta \gamma}^{3 / 2}\left(z_{1}, z_{2}, z_{3}\right)=\varepsilon^{i j k}\left(\not h \psi_{i}^{\uparrow}\right)_{\alpha}\left(z_{1} n\right)\left(\not h \psi_{j}^{\uparrow}\right)_{\beta}\left(z_{2} n\right)\left(\not h \psi_{k}^{\uparrow}\right)_{\gamma}\left(z_{3} n\right), \\
& B_{\alpha \beta \gamma}^{1 / 2}\left(z_{1}, z_{2}, z_{3}\right)=\varepsilon^{i j k}\left(\not h \psi_{i}^{\uparrow}\right)_{\alpha}\left(z_{1} n\right)\left(\not h \psi_{j}^{\downarrow}\right)_{\beta}\left(z_{2} n\right)\left(\not h \psi_{k}^{\uparrow}\right)_{\gamma}\left(z_{3} n\right),
\end{aligned}
$$

where $\alpha, \beta, \gamma$ are spinor and $i, j, k$ color indices and the superscript refers to the total helicity $\lambda=3 / 2$ or $\lambda=1 / 2$. The factors $\not h$ project onto the $\psi_{+}$ components of the four-component Dirac spinor fields

$$
\psi=\psi_{+}+\psi_{-} \equiv \frac{1}{2} \not h \not h \psi+\frac{1}{2} \not h \not h \psi,
$$

cf. Eq. (2.5). We will tacitly assume that the three quarks have different

\footnotetext{
$\overline{\mathrm{b}}$ We hope that using the same notation $\psi$ for the quark field and the Euler $\psi$-function will not create confusion.
} 
flavor. Identity of the quarks does not influence renormalization but rather introduces certain selection rules which pick up eigenstates with particular symmetries. The operators (2.6) and (2.7) appear in the QCD theory of hard exclusive processes. They are used to define the longitudinal momentum fraction distributions of quarks inside the $\Delta$-resonance and the proton, respectively.

Similarly to (2.5) the renormalized three-quark light-ray operators are defined through their Taylor expansion

$$
B\left(z_{1}, z_{2}, z_{3}\right)=\sum_{N} \sum_{k_{1}+k_{2}+k_{3}=N} \frac{z_{1}^{k_{1}}}{k_{1} !} \frac{z_{2}^{k_{2}}}{k_{2} !} \frac{z_{3}^{k_{3}}}{k_{3} !} D_{+}^{k_{1}} \psi(0) D_{+}^{k_{2}} \psi(0) D_{+}^{k_{3}} \psi(0) .
$$

Local three-quark operators having the same total number of derivatives all mix together but the ones containing total derivatives can be discarded by taking forward matrix elements. One is then left with a nontrivial mixing matrix of size $N+1$ for given $N$. Eigenvalues of this matrix $\gamma_{N, q}$ now have two indices: $N$, which refers to the total number of derivatives, and $q$, which enumerates the anomalous dimensions (e.g., from below) for a given $N$. In the case of $B^{3 / 2}$, we will be able to identify $q$ with a conserved charge.

The rest of this section is organized as follows. After a short exposition of the conformal symmetry in Sect. 2.1, we develop in Sect. 2.2 an approach to operator renormalization in which this symmetry becomes manifest. In this form the relation to spin-chain Hamiltonians also becomes apparent and this connection is elaborated in detail in Sect. 2.3. In Sect. 2.4, we use complete integrability to construct a semiclassical expansion of the anomalous dimensions $\gamma_{N, q}^{3 / 2}$ in powers of $1 / N$. In Sect. 2.5 , the difference between $B^{3 / 2}$ and $B^{1 / 2}$ is discussed and we find that breaking of integrability in the case of $B^{1 / 2}$ produces a mass gap in the spectrum of anomalous dimensions by a mechanism that can be interpreted as binding of quarks into scalar diquarks. Sect. 2.6 contains a short summary of other applications, in particular, the case of quark-antiquark-gluon operators that are related to open spin chains. In Sect. 2.7, we introduce the general notion of quasi-partonic operators which will be important for the discussion of supersymmetric extensions. Finally, in Sect. 2.8 we comment on the conformal symmetry breaking in QCD at higher orders.

\subsection{Conformal symmetry and the collinear subgroup}

Among the general coordinate transformations of the four-dimensional Minkowski space that conserve the interval $d s^{2}=g_{\mu \nu}(x) d x^{\mu} d x^{\nu}$, there are 
transformations that change only the scale of the metric:

$$
g_{\mu \nu}^{\prime}\left(x^{\prime}\right)=\omega(x) g_{\mu \nu}(x)
$$

and, consequently, preserve angles and leave the light-cone invariant. All transformations belonging to this subclass form, by definition, the conformal group. It is obvious that conformal transformations correspond to a generalization of the usual Poincaré group, since the Minkowski metric is not changed by translations and Lorentz rotations. Examples of specific conformal transformations are dilatations (global scale transformations) and inversion

$$
x^{\mu} \rightarrow x^{\prime \mu}=\lambda x^{\mu}, \quad x^{\mu} \rightarrow x^{\prime \mu}=\frac{x^{\mu}}{x^{2}},
$$

with real $\lambda$. Another important example is the so-called special conformal transformation

$$
x^{\mu} \rightarrow x^{\prime \mu}=\frac{x^{\mu}+a^{\mu} x^{2}}{1+2 a \cdot x+a^{2} x^{2}},
$$

which corresponds to the sequence of inversion, translation by an arbitrary constant vector $a_{\mu}$ and a further inversion with the same origin. The full conformal algebra in 4 dimensions includes fifteen generators: $\mathbf{P}_{\mu}$ (4 translations), $\mathbf{M}_{\mu \nu}$ (6 Lorentz rotations), $\mathbf{D}$ (dilatation) and $\mathbf{K}_{\mu}$ (4 special conformal transformations) and is a generalization of the well-known 10-parameter Lie algebra of the Poincaré group generated by $\mathbf{P}_{\mu}$ and $\mathbf{M}_{\mu \nu}$.

For fields "living" on the light-ray, only a few conformal transformations are relevant. One of them is a special case of the conformal transformation in Eq. (2.12), with $a_{\mu}$ being a light-like vector $a_{\mu}=a \bar{n}_{\mu}$. One finds

$$
x_{-} \rightarrow x_{-}^{\prime}=\frac{x_{-}}{1+2 a x_{-}}
$$

so that these transformations map a light-ray in the $x_{-}$-direction into itself. Together with the translations and dilatations in the same direction, $x_{-} \rightarrow$ $x_{-}+c$ and $x_{-} \rightarrow \lambda x_{-}$, they form a so-called collinear subgroup of the full conformal group. This subgroup is nothing but the familiar $S L(2, \mathbb{R})$ group of projective transformations on a line:

$$
\begin{aligned}
z \rightarrow z^{\prime} & =\frac{\alpha z+\beta}{\gamma z+\delta}, \quad \alpha \delta-\beta \gamma=1, \\
\Phi(z) \rightarrow \Phi^{\prime}(z) & =(\gamma z+\delta)^{-2 j} \Phi\left(\frac{\alpha z+\beta}{\gamma z+\delta}\right),
\end{aligned}
$$


where $\Phi$ is a generic quantum field (quark or gluon), $\alpha, \beta, \gamma, \delta$ are real numbers and $j$ is the conformal spin

$$
j=(d+s) / 2 .
$$

The parameter $d$ is called the scaling dimension and it specifies the field transformation under the dilatations

$$
\delta_{D} \Phi(x) \equiv i[\Phi(x), \mathbf{D}]=-(x \cdot \partial+d) \Phi(x) .
$$

In a free theory (i.e., at the classical level) the scaling dimension coincides with the canonical dimension $d^{\text {can }}$, which is fixed by the requirement that the action of the theory is dimensionless. In the quantum theory $d \neq d^{\text {can }}$, in general, and the difference is called the anomalous dimension. In turn, $s$ is the spin projection of $\Phi$ on the "+" direction:

$$
\Sigma_{-+} \Phi(z)=s \Phi(z) .
$$

Here we assume that the field $\Phi$ is chosen to be an eigenstate of the spin operator $\Sigma_{-+}$. In the general case, one should use suitable projection operators to separate different spin components.

The collinear conformal transformations are generated by the four generators $\mathbf{P}_{+}, \mathbf{M}_{-+}, \mathbf{D}$ and $\mathbf{K}_{-}$which form a subalgebra of the full conformal algebra. In order to bring the commutation relations to the standard form, it is convenient to introduce the following linear combinations: $\mathbf{L}_{+}=$ $\mathbf{L}_{1}+i \mathbf{L}_{2}=-i \mathbf{P}_{+}, \mathbf{L}_{-}=\mathbf{L}_{1}-i \mathbf{L}_{2}=(i / 2) \mathbf{K}_{-}$and $\mathbf{L}_{0}=(i / 2)\left(\mathbf{D}+\mathbf{M}_{-+}\right)$ The action of the generators on primary quantum fields can be traded for the algebra of differential operators acting on the field coordinates on the light cone

$$
\begin{aligned}
{\left[\mathbf{L}_{+}, \Phi(z)\right] } & =-\partial_{z} \Phi(z) \equiv L_{+} \Phi(z) \\
{\left[\mathbf{L}_{-}, \Phi(z)\right] } & =\left(z^{2} \partial_{z}+2 j z\right) \Phi(z) \equiv L_{-} \Phi(z) \\
{\left[\mathbf{L}_{0}, \Phi(z)\right] } & =\left(z \partial_{z}+j\right) \Phi(z) \equiv L_{0} \Phi(z)
\end{aligned}
$$

where $\partial_{z}=\partial / \partial z$. The differential operators $L_{i}$ satisfy the familiar $S L(2)$ commutation relations:

$$
\left[L_{0}, L_{\mp}\right]= \pm L_{\mp}, \quad\left[L_{-}, L_{+}\right]=2 L_{0} .
$$

The remaining generator $\mathbf{E}=(i / 2)\left(\mathbf{D}-\mathbf{M}_{-+}\right)$counts the so-called collinear twist of the field $\Phi:[\mathbf{E}, \Phi(z)]=\frac{1}{2}(d-s) \Phi(z)$. It commutes with all $\mathbf{L}_{i}$ and is not relevant for most of our discussions. 
A local field operator $\Phi(z)$ that has fixed spin projection (2.17) on the light-cone is an eigenstate of the quadratic Casimir operator

$$
\sum_{i=0,1,2}\left[\mathbf{L}_{i},\left[\mathbf{L}_{i}, \Phi(z)\right]\right]=j(j-1) \Phi(z)=L^{2} \Phi(z)
$$

with the operator $L^{2}$ defined as

$$
L^{2}=\sum_{i=0,1,2} L_{i}^{2}=\left(L_{0}\right)^{2}-L_{0}+L_{-} L_{+}, \quad\left[L^{2}, L_{i}\right]=0 .
$$

Eqs. (2.14) and (2.20) imply that the field $\Phi(z)$ is transformed under the projective transformations according to a representation of the $S L(2, \mathbb{R})$ group specified by the parameter $j$; hence the rationale of referring to it as the conformal spin of the field. We will see below that we are dealing with infinite-dimensional representations of the collinear conformal group. In general, different spin components of the fields have different conformal spin. For example, for the "good" components of the quark field, Eq. (2.8), $s=+1 / 2$ and the conformal spin takes the value $j_{q}=1$; for the transverse component of the gauge strength tensor $n^{\mu} F_{\mu \perp}$ the conformal spin equals $j_{g}=3 / 2$ and for the scalar field one has $j_{s}=1 / 2$.

Besides the collinear subgroup just described, one can consider another subgroup corresponding to transformations of the two-dimensional transverse plane $x_{\perp}^{\mu}=\left(0, x_{1}, x_{2}, 0\right)$, introduced in (2.4). This "transverse" subgroup is relevant for the Regge kinematics and will be considered in the corresponding section.

\subsection{Hamiltonian approach to operator renormalization}

In quantum theory, composite operators mix with each other under the renormalization group flow in the cut-off parameter. This flow is driven by the dilatation operator,

$$
[\mathcal{O}, \mathbf{D}]=i\left(d^{\text {can }}-\mu \frac{\partial}{\partial \mu}-\beta(g) \frac{\partial}{\partial g}\right) \mathcal{O},
$$

where $d_{\text {can }}$ is the canonical dimension of the composite operator $\mathcal{O}$. This equality is understood as an operator insertion into a Green function and is known as the Callan-Symanzik equation. Conformal symmetry of the QCD Lagrangian (for massless quarks) leads to important constraints on the above operator mixing. Indeed, the one-loop renormalization is governed by the infinite parts of the one-loop Feynman diagrams which have the symmetry of the classical Lagrangian. It follows that the QCD operators belonging to 


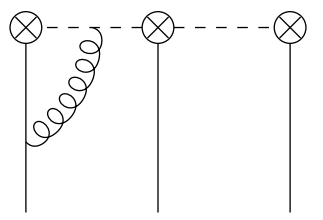

a

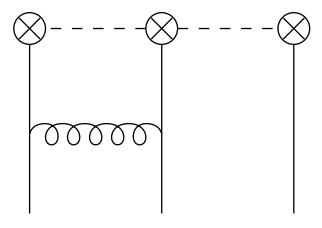

$\mathrm{b}$

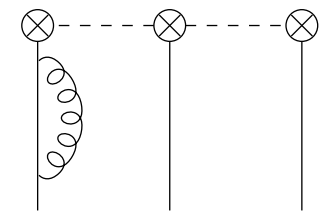

C

Figure 1. Examples of a "vertex" correction (a), "exchange" diagram (b) and self-energy insertion (c) contributing to the renormalization of three-quark operators in Feynman gauge. Path-ordered gauge factors are shown by the dashed lines. The set of all diagrams includes possible permutations.

different representations of the (collinear) conformal group cannot mix under renormalization at leading-logarithmic accuracy. This simplification proves to be crucial, e.g., for the QCD description of hadron form factors at very large momentum transfers. The aim of this section is, first, to explain this symmetry of the QCD renormalization group equations using a simple example and, second, to re-interpret these equations as a quantum-mechanical Hamiltonian problem.

To make the conformal symmetry explicit, it is convenient to work with light-ray operators (2.6), (2.7) directly, rather than decompose them in local operators. The renormalization group equations for light-ray operators can be written as [47-49]

$$
\left\{\mu \frac{\partial}{\partial \mu}+\beta(g) \frac{\partial}{\partial g}\right\} B=\mathbb{H} \cdot B,
$$

where $\mathbb{H}$ is a certain integral operator corresponding, to the one-loop accuracy, to contributions of the Feynman diagrams shown in Fig. 1.

To simplify notations we factor out the QCD coupling constant, the color factors and trivial contributions of the self-energy insertions: ${ }^{c}$

$$
\mathbb{H}=\frac{g^{2}}{8 \pi^{2}}\left[\left(1+1 / N_{c}\right) \mathcal{H}+3 C_{F} / 2\right]
$$

where $C_{F}=\left(N_{c}^{2}-1\right) /\left(2 N_{c}\right)$ is the Casimir operator in the fundamental rep-

\footnotetext{
${ }^{\mathrm{c}}$ The color factors of the diagrams shown in Figs. 1 $\mathrm{l}$ and $\mathrm{b}$ can be calculated using the identity $\varepsilon_{i^{\prime} j^{\prime} k} t_{i^{\prime} i}^{a} t_{j^{\prime} j}^{a}=-\varepsilon_{i j k}\left(1+1 / N_{c}\right) / 2$ with $t^{a}$ being the generators of the $S U\left(N_{c}\right)$ in the quark representation.
} 
resentation of $S U\left(N_{c}\right)$. In the Feynman gauge, the gluon exchange diagram in Fig. 1o vanishes unless the participating quarks have opposite helicity. The renormalization of the $\lambda=3 / 2$ operator $B_{3 / 2}$ (2.6) is therefore determined by the vertex correction in Fig. 10 alone. By explicit calculation one finds $[50,51]$

$$
\mathcal{H}_{3 / 2}=\mathcal{H}_{12}^{v}+\mathcal{H}_{23}^{v}+\mathcal{H}_{13}^{v},
$$

where $\mathcal{H}_{i k}^{v}$ are the two-particle kernels involving the $i$-th and $k$-th quarks, for example,

$$
\begin{array}{r}
\mathcal{H}_{12}^{v} B\left(z_{i}\right)=-\int_{0}^{1} \frac{d \alpha}{\alpha}\left\{\bar{\alpha}\left[B\left(z_{12}^{\alpha}, z_{2}, z_{3}\right)-B\left(z_{1}, z_{2}, z_{3}\right)\right]\right. \\
\left.+\bar{\alpha}\left[B\left(z_{1}, z_{21}^{\alpha}, z_{3}\right)-B\left(z_{1}, z_{2}, z_{3}\right)\right]\right\},
\end{array}
$$

with $\bar{\alpha} \equiv 1-\alpha$ and $z_{i k}^{\alpha} \equiv z_{i} \bar{\alpha}+z_{k} \alpha$.

In the case of $B_{1 / 2}$, the vertex correction remains the same, but one has to add the contributions of gluon exchange between the quarks with opposite helicity. One obtains

$$
\mathcal{H}_{1 / 2}=\mathcal{H}_{3 / 2}-\mathcal{H}_{12}^{e}-\mathcal{H}_{23}^{e},
$$

where we assume that the first and the third quark have the same helicity, as in Eq. (2.7). The kernels $H_{i k}^{e}$ act on $i$-th and $k$-th arguments of the nonlocal operators only, and can be written in the form

$$
\mathcal{H}_{12}^{e} B\left(z_{i}\right)=\int \mathcal{D} \alpha B\left(z_{12}^{\alpha_{1}}, z_{21}^{\alpha_{2}}, z_{3}\right),
$$

with the integration measure $\mathcal{D} \alpha$ defined as

$$
\int \mathcal{D} \alpha \equiv \int_{0}^{1} d \alpha_{1} d \alpha_{2} d \alpha_{3} \delta\left(1-\alpha_{1}-\alpha_{2}-\alpha_{3}\right) .
$$

The expected conformal invariance of the evolution equation for baryonic operators implies that the two-particle kernels $\mathcal{H}_{i k}$ commute with the generators of the $S L(2)$ transformations $L_{\alpha}$ defined in (2.18). To show this, consider the following expression that generalizes both (2.26) and (2.28):

$$
\mathcal{H}_{12} B\left(z_{1}, z_{2}, z_{3}\right)=\int \mathcal{D} \alpha \omega\left(\alpha_{1}, \alpha_{2}\right) B\left(z_{1}-\alpha_{1} z_{12}, z_{2}+\alpha_{2} z_{12}, z_{3}\right),
$$

where $z_{12}=z_{1}-z_{2}$. The integration measure is defined in (2.29). This operator has a simple meaning: acting on the three-particle nonlocal operator $B\left(z_{1}, z_{2}, z_{3}\right)$ it displaces the quarks with the coordinates $z_{1}$ and $z_{2}$ on the light-cone in the direction of each other. The vertex correction in (2.26) 
is obtained with $\omega^{v}\left(\alpha_{1}, \alpha_{2}\right)=\delta\left(\frac{\alpha_{1} \alpha_{2}}{\bar{\alpha}_{1} \bar{\alpha}_{2}}\right)$ and the exchange contribution in (2.26) corresponds to $\omega^{e}\left(\alpha_{1}, \alpha_{2}\right)=1$.

It is easy to see that, for this ansatz, $\left[\mathcal{H}_{12}, L_{+}\right]=\left[\mathcal{H}_{12}, L_{0}\right]=0$ for an arbitrary function $\omega\left(\alpha_{1}, \alpha_{2}\right)$, whereas the condition $\left[\mathcal{H}_{12}, L_{-}\right]=0$ leads to the following constraint:

$$
\left(\frac{\partial}{\partial \alpha_{1}} \alpha_{1} \bar{\alpha}_{1}+2 \alpha_{1} j_{1}\right) \omega\left(\alpha_{1}, \alpha_{2}\right)=\left(\frac{\partial}{\partial \alpha_{2}} \alpha_{2} \bar{\alpha}_{2}+2 \alpha_{2} j_{2}\right) \omega\left(\alpha_{1}, \alpha_{2}\right)
$$

where $\bar{\alpha}=1-\alpha$. Its general solution has the form

$$
\omega\left(\alpha_{1}, \alpha_{2}\right)=\bar{\alpha}_{1}^{2 j_{1}-2} \bar{\alpha}_{2}^{2 j_{2}-2} \varphi\left(\frac{\alpha_{1} \alpha_{2}}{\bar{\alpha}_{1} \bar{\alpha}_{2}}\right)
$$

with an arbitrary $\varphi$. Note that the conformal spins $j_{k}=1$ for all the three quark fields entering (2.6) and (2.7) so that the prefactor $\bar{\alpha}_{1}^{2 j_{1}-2} \bar{\alpha}_{2}^{2 j_{2}-2}$ in (2.32) disappears and the conformal symmetry requires that the kernel $\omega\left(\alpha_{1}, \alpha_{2}\right)$ is an arbitrary function of the ratio $\frac{\alpha_{1} \alpha_{2}}{\bar{\alpha}_{1} \bar{\alpha}_{2}}$ which is indeed the case for both expressions (2.26) and (2.28).

Once conformal symmetry of the two-particle kernels is established, the group theory tells us that $\mathcal{H}_{i k}$ may only depend on the corresponding twoparticle Casimir operators $L_{i k}^{2}$. To find the functional form of this dependence one has to compare their action on a suitable basis of trial functions. For definiteness, let us find $\mathcal{H}_{12}$ as a function of $L_{12}^{2}$. To this end, it is enough to compare their action on the homogeneous polynomials of two variables $z_{1}$ and $z_{2}$ :

$$
B\left(z_{1}, z_{2}, z_{3}\right) \longrightarrow b_{n}\left(z_{1}, z_{2}\right)
$$

which we choose to be eigenfunctions of the operator

$$
L_{12}^{2}=-\partial_{1} \partial_{2} z_{12}^{2} ; \quad z_{12} \equiv z_{1}-z_{2} .
$$

It is easy to see that the polynomials defined thus form an infinitedimensional representation of the $S L(2)$ group on which the operators $L_{+} \equiv L_{1,+}+L_{2,+}$ and $L_{-} \equiv L_{1,-}+L_{2,-}$ act as the lowering and raising operators respectively. It is thus sufficient to consider only the functions (polynomials) annihilated by $L_{+}$, or equivalently, the highest weight of the representation, since all other eigenfunctions of $L_{12}^{2}$ can then be obtained by a repeated application of $L_{-}$. Since $L_{+}=-\left(\partial_{1}+\partial_{2}\right)$, the latter condition is simply translation invariance, which leaves one with

$$
b\left(z_{1}, z_{2}\right)=\left(z_{1}-z_{2}\right)^{n} \equiv z_{12}^{n}, \quad n=0,1,2, \ldots
$$


An explicit calculation gives

$$
\begin{aligned}
L_{12}^{2} z_{12}^{n} & =(n+2)(n+1) z_{12}^{n}, \\
\mathcal{H}_{12}^{v} z_{12}^{n} & =2[\psi(n+2)-\psi(2)] z_{12}^{n}, \\
\mathcal{H}_{12}^{e} z_{12}^{n} & =1 /[(n+2)(n+1)] z_{12}^{n},
\end{aligned}
$$

where $\psi(x)=d \ln \Gamma(x) / d x$ is the Euler $\psi$-function. To cast (2.35) in an operator form, define $J_{12}$ as a formal solution of the operator relation

$$
L_{12}^{2}=J_{12}\left(J_{12}-1\right) .
$$

The eigenvalues of $J_{12}$ equal $j_{12}=n+2$ and specify the possible values of the sum of two $j=1$ conformal spins of quarks in the (12)-pair, cf. (2.21). Then

$$
\begin{aligned}
& \mathcal{H}_{12}^{v}=2\left[\psi\left(J_{12}\right)-\psi(2)\right], \\
& \mathcal{H}_{12}^{e}=1 /\left[J_{12}\left(J_{12}-1\right)\right]=1 / L_{12}^{2} .
\end{aligned}
$$

Substituting the representation (2.37) into (2.25) and (2.27) one obtains a Schrödinger equation for three particles with the coordinates $z_{1}, z_{2}$ and $z_{3}$ on the light-ray line. The 'Hamiltonians' $\mathcal{H}_{3 / 2}$ and $\mathcal{H}_{1 / 2}$ entering this equation for different baryon states have a pairwise structure and are expressed in terms of the corresponding two-particle Casimir operators (2.37).

The precise form of the Schrödinger equation and the meaning of the corresponding "wave function" depend on the representation that is used for the $S L(2)$ generators. Looking for polynomial solutions in light-cone coordinates $\Phi\left(z_{1}, z_{2}, z_{3}\right)$ as in (2.34) is one possibility:

$$
\mathcal{H} \cdot \Phi_{N, q}=\mathcal{E}_{N, q} \Phi_{N, q} \cdot
$$

Here $N$ refers to the degree of the polynomial alias the total number of derivatives, and $q$ enumerates the energy levels.

Another option is to go over to local operators. A generic local operator with $N$ derivatives can be written as the sum of monomials entering the expansion (2.9) with arbitrary coefficients

$$
\mathcal{O}=\sum_{k_{1}+k_{2}+k_{3}=N} c_{k_{1}, k_{2}, k_{3}} D_{+}^{k_{1}} \psi(0) D_{+}^{k_{2}} \psi(0) D_{+}^{k_{3}} \psi(0),
$$

and can be represented by a polynomial in three variables

$$
\Psi\left(x_{1}, x_{2}, x_{3}\right)=\sum_{k_{1}+k_{2}+k_{3}=N} c_{k_{1} k_{2} k_{3}} x_{1}^{k_{1}} x_{2}^{k_{2}} x_{3}^{k_{3}} .
$$


Note that $\Psi\left(x_{i}\right)$ serves as a projector, separating out the contribution of the local operator $O_{\Psi}$ to the nonlocal operator $B\left(z_{i}\right)$, which can be made explicit by writing

$$
\mathcal{O}_{\Psi}=\left.\Psi\left(\partial_{1}, \partial_{2}, \partial_{3}\right) B\left(z_{1}, z_{2}, z_{3}\right)\right|_{z_{i}=0}
$$

Therefore, one can call $\Psi\left(x_{i}\right)$ the coefficient function of a local operator. Since a local operator is completely determined by its coefficient function, diagonalization of the mixing matrix for operators can be reformulated as diagonalization of the mixing matrix for the coefficient functions. Requiring that $\mathcal{O}_{\Psi}$ (2.41) is multiplicatively renormalized, one ends up with a matrix equation in the space of homogeneous polynomials of degree $N$ of three variables

$$
\mathcal{H} \cdot \Psi_{N, q}=\mathcal{E}_{N, q} \Psi_{N, q}
$$

where the Hamiltonian $\mathcal{H}$ is given by the same expression, Eq. (2.37), in terms of the two-particle Casimir operators, but the $S L(2)$ generators have to be taken in a different, adjoint representation (see $[18,22]$ for details). The eigenvalues of $\mathcal{H}$ in (2.38) and (2.42) are of course the same; they correspond to the anomalous dimensions

$$
\gamma_{N, q} \equiv \frac{\alpha_{s}}{2 \pi}\left[\left(1+1 / N_{c}\right) \mathcal{E}_{N, q}+3 / 2 C_{F}\right] .
$$

The wave functions in (2.38) and (2.42) are related by a certain integral transformation, see [18]. The advantage of using $\Phi\left(z_{i}\right)$ is that is this representation the highest weight vector condition $L_{+} \Phi\left(z_{i}\right)=0$ reduces to the requirement of the translation invariance of $\Phi\left(z_{i}\right)=0$. On the contrary, the corresponding equation for the operator coefficient functions is more complicated (see also [52]).

The Hamiltonian in (2.42) is hermitian with respect to the conformal scalar product [16]. This implies that the anomalous dimensions take real quantized values and the corresponding eigenfunctions are mutually orthogonal with the weight function $x_{1} x_{2} x_{3}$

$$
\int \mathcal{D} x x_{1} x_{2} x_{3} \Psi_{N, q}\left(x_{i}\right) \Psi_{N, q^{\prime}}\left(x_{i}\right) \sim \delta_{q, q^{\prime}}
$$

The construction that we have described was first suggested in [12]. It is general and can be used in all other cases. 


\subsection{Complete integrability and non-compact Heisenberg magnets}

The Hamiltonian $\mathcal{H}_{3 / 2}$ possesses an additional "hidden" symmetry. One can construct an integral of motion (conserved charge) that commutes with $\mathcal{H}_{3 / 2}$ and with the $S L(2)$ generators:

$$
\begin{gathered}
\mathcal{Q}=\frac{i}{2}\left[L_{12}^{2}, L_{23}^{2}\right]=i^{3} \partial_{1} \partial_{2} \partial_{3} z_{12} z_{23} z_{31}, \\
{\left[\mathcal{Q}, L_{\alpha}\right]=\left[\mathcal{Q}, \mathcal{H}_{3 / 2}\right]=0,}
\end{gathered}
$$

where the explicit expression in the first line refers to the representation of the generators in Eq. (2.18). The existence of a non-trivial integral of motion makes the corresponding Schrödinger equation (2.38) completely integrable. Indeed, Eq. (2.45) tells us that $\mathcal{H}_{3 / 2}, \mathcal{Q}$, the total three-quark conformal spin $L^{2}=\sum_{i=0,1,2}\left(L_{1, i}+L_{2, i}+L_{3, i}\right)^{2}$ and its projection $L_{0}=L_{1,0}+L_{2,0}+L_{3,0}$ can be diagonalized simultaneously. Since there are only three independent degrees of freedom, this implies that the Hamiltonian $\mathcal{H}_{3 / 2}$ is a (complicated) function of the conserved charges. As a reward, one can replace the Schrödinger equation by a simpler problem of diagonalization of the conserved charge $\mathcal{Q}$. The eigenvalues of $\mathcal{Q}$ provide one with a "hidden" quantum number that can be used to parameterize the spectrum of the Hamiltonian, $\mathcal{E}_{3 / 2}=\mathcal{E}_{3 / 2}(N, q){ }^{\mathrm{d}}$

It turns out that $\mathcal{H}_{3 / 2}$ can be viewed as the Hamiltonian of the noncompact $S L(2, \mathbb{R})$ Heisenberg spin magnet. As a hint, notice that the expression for the Hamiltonian $\mathcal{H}_{3 / 2}$ as the sum of the Euler $\psi$-functions, Eqs. (2.25) and (2.37), resembles the definition of generalized $S U(2)$ Heisenberg magnet, Eqs. (1.2) and (1.3). The important difference between the two expressions is, however, that the two-particle "spin" $J_{12}$ in (2.37) takes an infinite set of quantized values $J_{12}=n+2$ with $n \geq 0$, while for the same operator in (1.2) one has $0 \leq J_{12} \leq 2 s$. In other words, in these two cases we deal with the infinite-dimensional representations of the $S L(2, \mathbb{R})$ group and finite-dimensional representation of the $S U(2)$ group, respectively.

To establish the exact correspondence, we can think of three quark operators inside $B\left(z_{1}, z_{2}, z_{3}\right)$ as three sites of the lattice and reinterpret the generators of the collinear $S L(2, \mathbb{R})$ subgroup, $L_{0}, L_{+}$and $L_{-}$, acting on

\footnotetext{
${ }^{\mathrm{d}}$ For the local conformal operator with the coefficient function $\Psi\left(x_{i}\right)$ that satisfies the highest weight condition $L_{+} \Psi\left(x_{i}\right)=0$ one finds $L_{0} \Psi\left(x_{i}\right)=J \Psi\left(x_{i}\right)$ and $L^{2} \Psi\left(x_{i}\right)=J(J-1) \Psi\left(x_{i}\right)$. Here $J=N+3 j$ is the total conformal spin with $j=1$ being the conformal spin of the quark field. Hence the dependence of the energy eigenvalues on the conformal spin (and spin projection) can be traded for the dependence on $N$, the total number of derivatives.
} 
$k$-th quark field as defining the spin operators in $k$-th site

$$
S_{k}^{+}=z_{k}^{2} \partial_{k}-2 s z_{k}, \quad S_{k}^{-}=-\partial_{k}, \quad S_{k}^{3}=z_{k} \partial_{k}-s,
$$

with $S^{ \pm}=S^{1} \pm i S^{2}$ and spin $s$ related to the conformal spin of the quark field $s=-j=-1$.

The spin operators (2.46) are the generators of a unitary representation of the $S L(2, \mathbb{R})$ group of the discrete series. The definition of the integrable spin chain is based on the existence of a fundamental operator, the $R$-matrix, $R_{k m}(u)$, which acts on the coordinates in sites $k$ and $m$, depends on an arbitrary complex parameter $u$ and satisfies the Yang-Baxter equation [32]

$$
R_{12}(u) R_{13}(v) R_{23}(u-v)=R_{23}(u-v) R_{13}(v) R_{12}(u),
$$

with $u$ and $v$ being arbitrary complex spectral parameters. For infinitedimensional representations of the $S L(2, \mathbb{R})$ group of the discrete series, the solution to Eq. (2.47) is given by [3]

$$
R_{k m}(u)=(-1)^{J_{k m}} \frac{\Gamma\left(J_{k m}-i u\right)}{\Gamma\left(J_{k m}+i u\right)},
$$

where $J_{k m}$ is the two-particle spin operator, cf. Eq. (2.36). The Hamiltonian of the $S L(2, \mathbb{R})$ Heisenberg magnet of length $L$ is defined in terms of the $R$-matrix as [4]

$$
\mathcal{H}_{\mathrm{xxx}}=\sum_{n=1}^{L} H_{n, n+1}, \quad H_{n, n+1}=-\left.i \frac{d}{d u} \ln R_{n, n+1}(u)\right|_{u=0},
$$

with $H_{L, L+1} \equiv H_{L, 1}$. It is easy to verify that for $L=3$ the Hamiltonian (2.49) coincides with $\mathcal{H}_{3 / 2}$ up to an irrelevant additive constant.

Complete integrability of the Hamiltonian $\mathcal{H}_{3 / 2}$ allows one to solve the Schrödinger equation by applying the Bethe Ansatz. The exact expression for the energy spectrum is given by

$$
\mathcal{E}_{N, q}=\left.i \frac{d}{d u} \ln \frac{Q(u+i j)}{Q(u-i j)}\right|_{u=0},
$$

where the function $Q(u)$ is the eigenvalue of the Baxter operator for the $S L(2, \mathbb{R})$ magnet [53]. It satisfies the finite-difference Baxter equation

$$
t(u) Q(u)=(u+i j)^{3} Q(u+i)+(u-i j)^{3} Q(u-i)
$$

with the transfer matrix

$$
t(u)=2 u^{3}-(N+3 j)(N+3 j-1) u+q
$$


and $j=1$ being the conformal spin of the quark field, under the additional condition that $Q(u)$ is polynomial in the spectral parameter. These conditions determine $Q(u)$ up to an overall normalization and allow one to establish the quantization conditions for the charge $q$. The polynomial $Q(u)$ can be parameterized by its roots

$$
Q(u)=\prod_{j=1}^{N}\left(u-\lambda_{j}\right)
$$

with non-negative $N$ related to the total $S L(2, \mathbb{R})$ spin of the magnet $J=$ $N+3 j$. Substituting this expression into the Baxter equation (2.51), one finds that the roots $\lambda_{j}$ satisfy the Bethe equations for spin $s=-1$. The fact that the spin is negative leads to a number of drastic differences compared to conventional "compact" $S U(2)$ magnets. In particular, the Bethe roots take real values only and the number of solutions is infinite [9].

Solving the Baxter equation (2.51) for $N=0,1, \ldots$ one can reconstruct the spectrum of the Hamiltonian $\mathcal{H}_{3 / 2}$ and, as a consequence, determine the exact spectrum of the anomalous dimensions of the maximal helicity baryon operators, see Fig. 2]

We would like to stress that the function $Q(u)$ has profound physical meaning: It defines the eigenstates of the $S L(2, \mathbb{R})$ magnet in the representation of the Separated Variables [54]. Due to complete integrability of the Hamiltonian $\mathcal{H}_{3 / 2}$, its eigenfunctions $\Psi_{N, q}\left(z_{1}, z_{2}, z_{3}\right)$ have to diagonalize the conserved charge $\mathcal{Q}$ defined in (2.45). In general, the solutions to the differential equation $\mathcal{Q} \Psi_{N, q}\left(z_{1}, z_{2}, z_{3}\right)=q \Psi_{N, q}\left(z_{1}, z_{2}, z_{3}\right)$ are complicated functions of the light-cone variables $z_{k}$. Obviously, the spectrum of $\mathcal{H}_{3 / 2}$ and $\mathcal{Q}$ does not depend on the representation in which these operators are defined. The main idea behind the method of Separated Variables is to perform a unitary transformation on the Hilbert space of $\mathcal{H}_{3 / 2}$

$$
\mathcal{H}_{3 / 2} \rightarrow \mathcal{H}_{3 / 2}^{(\mathrm{SoV})}=U \mathcal{H}_{3 / 2} U^{\dagger}, \quad \Psi_{N, q} \rightarrow \Psi_{N, q}^{(\mathrm{SoV})}=U \Psi_{N, q}
$$

in order to go over to a representation in which the eigenfunction $\Psi_{N, q}^{(\mathrm{SoV})}$ is factorized into a product of functions depending on a single variable. Denoting the coordinates in the $\mathrm{SoV}$ representation as

$$
\left(z_{1}, z_{2}, z_{3}\right) \stackrel{\mathrm{SoV}}{\longrightarrow}\left(z_{0}, x_{1}, x_{2}\right)
$$

with $z_{0}$ being the center-of-mass coordinate of the system of three quarks on 

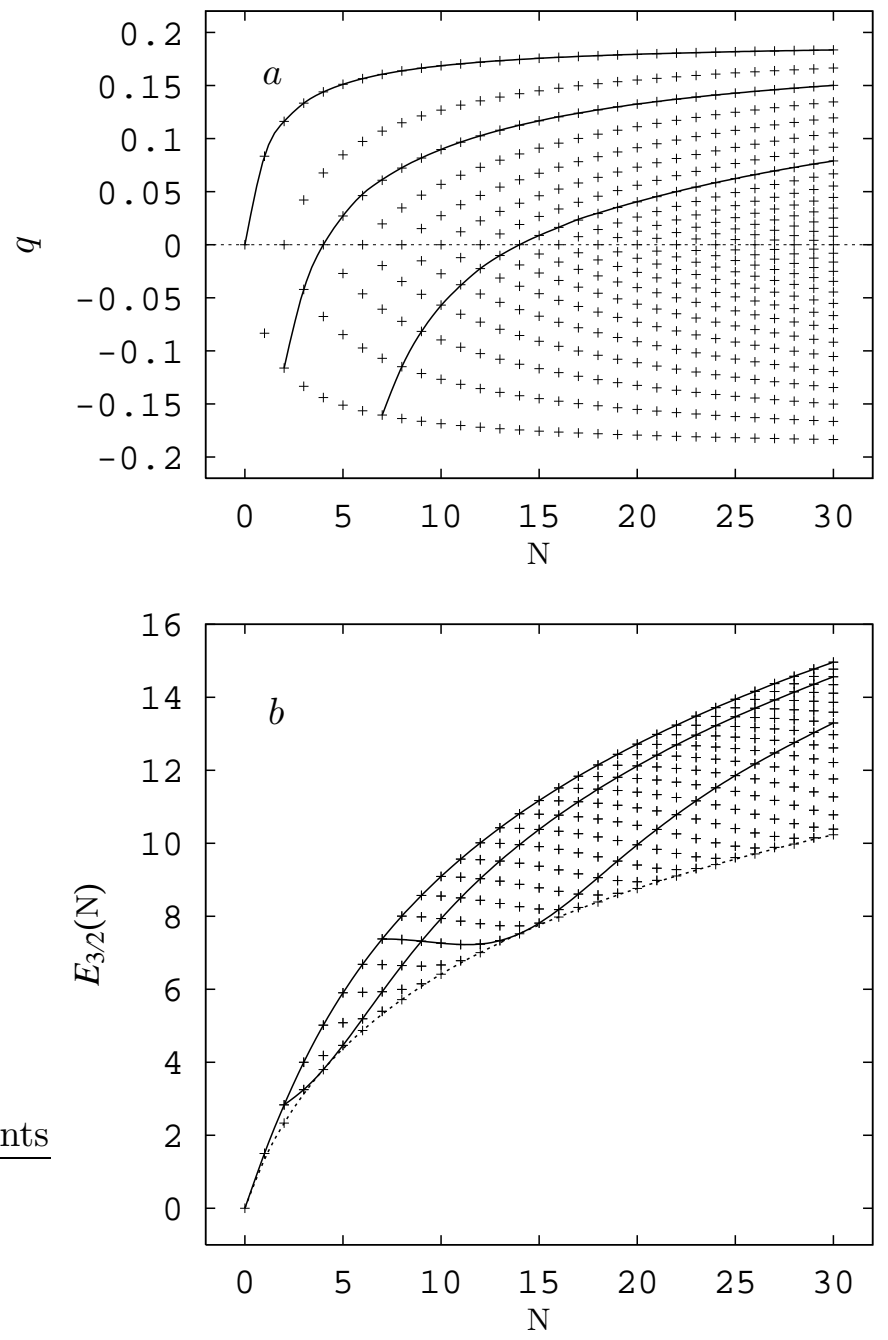

Figure 2. The spectrum of eigenvalues for the conserved charge, $q$ in $(a)$ and for the helicity-3/2 Hamiltonian, $\mathcal{E}_{N, q}$ in $(b)$.

the light-cone and $x_{1,2}$ being new collective coordinates, one has

$$
\Psi_{N, q}^{(\mathrm{SoV})}=\mathrm{e}^{i p z_{0}} Q\left(x_{1}\right) Q\left(x_{2}\right) .
$$

The explicit form of the unitary transformation to the Separated Variables, Eqs. (2.54) - 2.56), was found in [55]. Here $p$ is the total (light-cone) momentum of three quarks $\sum_{k=1}^{3} i \partial_{z_{k}} \Psi_{N, q}\left(z_{1}, z_{2}, z_{3}\right)=p \Psi_{N, q}\left(z_{1}, z_{2}, z_{3}\right)$, or equivalently the "-" component of the total momentum of the $S L(2, \mathbb{R})$ spin 
chain. The function $Q(x)$ defines the wave function in the separated variables, Eq. (2.56). In this way the Schrödinger equation for $\Psi_{N, q}$ is translated into the Baxter relation for $Q(x)$, Eq. (2.51). We conclude that the Baxter equation encodes the complete information about the system - its solutions $Q(x)$ govern the energy spectrum, Eq. (2.50), and define the wave functions in the representation of Separated Variables, Eq. (2.56).

\subsection{Semiclassical expansion}

The spectrum of the anomalous dimensions shown in Fig. 2 exhibits remarkable regularity. One can reveal its origin by making use of the interpretation of $Q(u)$ as the wave function in the separated variable. Assuming a parametrization $Q(x)=\exp \left(\frac{i}{\hbar} S(x)\right)$, one can solve the Baxter equation (2.51) by making a semiclassical expansion for $\hbar \rightarrow 0$. Along this line of reasoning, the rôle of the Planck constant is played by the (inverse) total conformal spin $1 / J=1 /(N+3 j)$ for $N \gg 1$.

To be more precise, we introduce a small parameter $\eta$

$$
\eta=[J(J-1)]^{-1 / 2}, \quad J=N+3 j
$$

and replace $u=x / \eta$ as the parameter in Eq. (2.51). Introducing the notation $f(x)$ for the rescaled $Q$-function

$$
f(x)=\eta^{J}(x / \eta)^{3} Q(x / \eta), \quad \widehat{q}=q \eta^{3} .
$$

the Baxter equation (2.51) takes the form

$$
f(x+i \eta)+f(x-i \eta)=x^{-3} t(x) f(x),
$$

where in the expression (2.52) for the transfer matrix $t(x)$, the parameter $q$ is replaced by $\widehat{q}$ defined in (2.58). We now look for the solutions of the rescaled Baxter equation in the form of the WKB expansion

$$
f(x)=\exp \left(\frac{i}{\eta} S_{0}(x)+i S_{1}(x)+\ldots\right),
$$

where each term is assumed to be uniformly bounded and the expansion is assumed to be convergent. Substituting the ansatz (2.60) in Eq. (2.59) and expanding both sides to order $\eta$, one obtains [17]

$$
2 \cosh S_{0}^{\prime}(x)=t(x) / x^{3}, \quad S_{1}^{\prime}(x)=\frac{i}{2} S_{0}^{\prime \prime}(x) \operatorname{coth} S_{0}^{\prime}(x),
$$


where a prime denotes a derivative with respect to $x$. To this accuracy, the solution of the Baxter equation (2.59) is given by

$$
f(x)=\frac{1}{\left[\sinh S_{0}^{\prime}(x)\right]^{1 / 2}} \exp \left(\frac{i}{\eta} \int^{x} d S_{0}(x)+\mathcal{O}(\eta)\right) .
$$

As usual, the leading term $S_{0}(x)$ defines the classical action function of the underlying quantum system. Introducing the notation

$$
\omega(x)=\exp \left(S_{0}^{\prime}(x)\right)=\exp (p(x))
$$

one can rewrite the first equation in (2.61) as

$$
\omega+\frac{1}{\omega}=x^{-3} t(x) .
$$

This equation establishes the relation between the classical momentum $p$ and the coordinate $x$ for "equal energy levels" defined by the charge $q$. Taking into account that $\omega$ is real on classical trajectories, we obtain the conditions that define the classically allowed regions of motion for quarks as

$$
t^{2}\left(x_{j}\right) \geq 4 x_{j}^{6}, \quad(j=1,2),
$$

where the subscript refers to the $j$-th allowed band.

Next, we continue the relation (2.64) into the complex domain, define $y=x^{N}\left(\omega-\frac{1}{\omega}\right)$ and rewrite (2.64) as [17]

$$
\Gamma: \quad y^{2}=t^{2}(x)-4 x^{6}=(-x+\widehat{q})\left(4 x^{3}-x+\widehat{q}\right),
$$

where $t(x)=2 x^{3}-x+\widehat{q}$ is a polynomial depending on the charge $\widehat{q}$. The curve (2.66) determines an elliptic Riemann surface $\Gamma$ equipped with a meromorphic differential $d S_{0}=p(x) d x$ defined in (2.61). Integrating this differential along some path that terminates at $x$ one obtains the "action" function $\int^{x} d S_{0}=\int^{x} p(x) d x$. In general, for arbitrary values of the conserved charge $q$, this function is single-valued on the Riemann surface but double-valued on the complex $x$-plane. We recall that the same "action" function defines the wave function in the separated coordinates, Eq. (2.62), which has to be a single-valued function on the real $x$-axis. To satisfy this requirement, the charge $q$ has to satisfy certain quantization conditions. In the semiclassical approach, they take the form of standard Bohr-Sommerfeld quantization conditions imposed on the solution in Eq. (2.62)

$$
a_{k} \equiv \oint_{\alpha_{k}} d S_{0}=2 \pi \eta\left(n_{k}+\frac{1}{2}\right)+O\left(\eta^{2}\right)
$$


with $k=1,2$ and $n_{k}$ being integer, such that $n_{1}+n_{2}=N$. Here the integration goes over the closed contours $\alpha_{k}$ on the Riemann surface encircling the intervals of the classical motion on the real $x$-axis, Eq. (2.65). Solving (2.67), one finds the spectrum of quantized charges

$$
q\left(N, n_{1}\right)=\frac{\eta^{-3}}{\sqrt{27}}\left[1-3 \eta\left(n_{1}+\frac{1}{2}\right)+\eta^{2}\left(2 n_{1}^{2}+2 n_{1}-\frac{13}{24}\right)+\ldots\right],
$$

with $\eta$ defined in (2.57). The integer $n_{1}$ parameterizes different "trajectories" as shown in Fig. 2, Note that all non-zero eigenvalues of $\mathcal{Q}$ come in pairs: If $q$ is an eigenvalue, then $-q$ is also an eigenvalue. As a consequence of the symmetry of quantization conditions in the separated coordinates, the trajectories $q\left(N, n_{1}\right)$ have the reflection symmetry $q\left(N, n_{1}\right)=-q\left(N, N-n_{1}\right)$, which maps positive values of $q$ on the $n_{1}$-th trajectory into the negative $q$ on the $n_{2}=\left(N-n_{1}\right)$-th trajectory.

Finally, the energy eigenvalues $\mathcal{E}_{N, q}$ can be calculated as a function of the spin $N$ and the charge $q$ with the help of Eq. (2.50). At the semiclassical level, one obtains

$$
\mathcal{E}_{N, q}=2 \ln 2-6+6 \gamma_{\mathrm{E}}+2 \Re \mathrm{e} \sum_{k=1}^{3} \psi\left(1+i \delta_{k} / \eta^{3}\right)+\mathcal{O}\left(\eta^{6}\right)
$$

where $\delta_{k}$ are the roots of the transfer matrix $t_{3}\left(\delta_{k}\right)=2 \delta_{k}^{3}-\delta_{k}+\widehat{q}=0$. Combined together, Eqs. (2.68) and (2.69) provide a good quantitative description of the spectrum of the anomalous dimensions for helicity-3/2 baryon operators. Note that $\mathcal{E}_{N, q}=\mathcal{E}_{N,-q}$ so that the anomalous dimensions are double degenerate, apart from the lowest eigenvalues for even $N$ which correspond to $q=0$. The origin of this degeneracy can be traced to the permutation symmetry between the three quarks [18].

\subsection{Breakdown of integrability}

There is no reason to expect that QCD is an integrable theory, simply because of the complexity of phenomena that it is supposed to describe. This complexity is certainly related to breaking of the conformal symmetry, but even so breaking of integrability in cases where the conformal symmetry is preserved may lead to important clues. The renormalization of the threequark operator $B^{1 / 2}$ provides one with a very instructive example of what can happen. As mentioned above, the operators $B^{3 / 2}$ (integrable) and $B^{1 / 2}$ (non-integrable) are related to quark distributions at small transverse separations inside the $\Delta$-isobar and the nucleon, respectively, and it is natural to speculate that the difference in light-cone dynamics in these two hadrons 


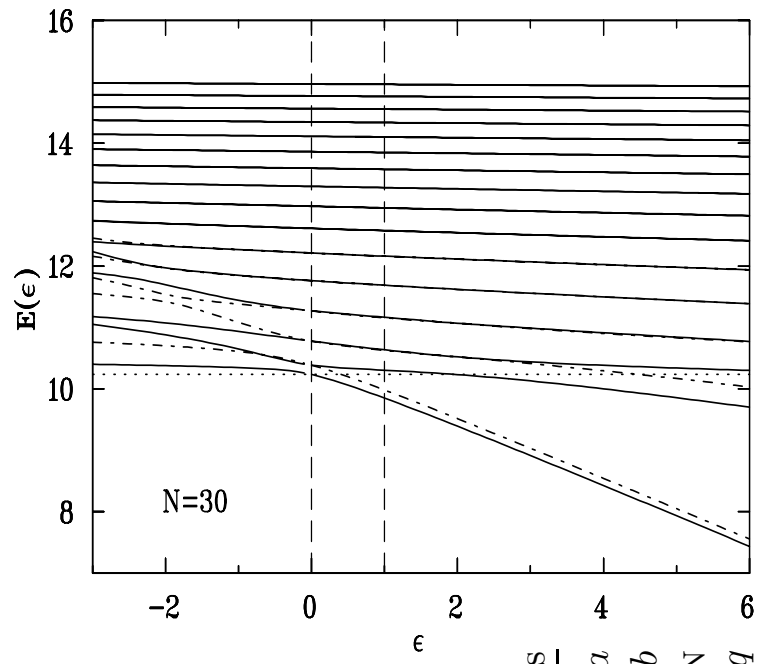

Figure 3. The flow of energy eigenvalues for the Hamiltonian $\mathcal{H}(\epsilon)$ for $N=30$ (see text). The solid and the dash-dotted curves show the parity-even and parity-odd levels, respectively. The two vertical dashed lines indicate $\mathcal{H}_{3 / 2} \equiv \mathcal{H}(\epsilon=0)$ and $\mathcal{H}_{1 / 2} \equiv \mathcal{H}(\epsilon=1)$, respectively. The horizontal dotted line shows position of the "ground state" that corresponds to $q=0$.

is related to the loss of integrability in the latter case. Let us study this difference in some detail.

The scale dependence of $B^{1 / 2}$ is driven by the Hamiltonian $\mathcal{H}_{1 / 2}$ defined in (2.27), which differs from $\mathcal{H}_{3 / 2}$ by the two terms corresponding to gluon exchange between quarks of opposite chirality (see Fig. 11):

$$
\mathcal{H}_{1 / 2}=\mathcal{H}_{3 / 2}+V, \quad V=-\left(\frac{1}{L_{12}^{2}}+\frac{1}{L_{23}^{2}}\right) .
$$

To visualize the effects of this additional interaction it proves to be convenient to consider a somewhat more general Hamiltonian

$$
\mathcal{H}(\epsilon)=\mathcal{H}_{3 / 2}+\epsilon V,
$$

with $\epsilon$ being a new coupling constant. $\mathcal{H}(\epsilon=0)$ reproduces $\mathcal{H}_{3 / 2}$ whereas $\mathcal{H}(\epsilon=1)$ coincides with the Hamiltonian $\mathcal{H}_{1 / 2}$. Thus, the spectra of $\mathcal{H}_{3 / 2}$ and $\mathcal{H}_{1 / 2}$ are related to each other through the flow of the energy levels of $\mathcal{H}(\epsilon)$ from $\epsilon=0$ to $\epsilon=1$ (see Fig. 3). Note that for $\epsilon \neq 0$, the cyclic permutation symmetry between the three quarks is also lost, but $\mathcal{H}(\epsilon)$ is still invariant under permutations of the first and the third quarks; $\left[\mathcal{H}(\epsilon), \mathcal{P}_{13}\right]=0$. As a result, the degeneracy between parity-odd and parity-even eigenstates is lifted and, in fact, the flows of levels with different parity are completely 
independent from one another.

The spectra in Fig. 3 exhibit the following characteristic features:

- In the upper part of the spectrum the effect of $\epsilon$-proportional terms on the spectrum of the 'unperturbed' Hamiltonian $\mathcal{H}(\epsilon=0)=\mathcal{H}_{3 / 2}$ is very mild. While at $\epsilon=0$ the energy levels are double degenerate, their splitting at $\epsilon \neq 0$ remains (exponentially, as one can show [18]) small for large $N$.

- For $\epsilon>0$, the two lowest levels are decoupled from the rest of the spectrum and fall off with $\epsilon$ almost linearly. In the large $-N$ limit they become separated from the rest of the spectrum by a finite "mass gap".

This structure suggests that the difference $\epsilon V=\mathcal{H}(\epsilon)-\mathcal{H}(\epsilon=0)$ can be considered as a perturbation for most of the levels, but not for the few lowest ones (for large $N$ ). Moreover, for $\epsilon>0$, which corresponds to attractive interaction, we seemingly witness creation of a certain bound state.

To formalize the argument, one has to evaluate the matrix elements of $V$ between the eigenstates of $\mathcal{H}_{3 / 2}$ and compare them with the energy splittings for the "unperturbed" Hamiltonian. By explicit calculation one confirms that the perturbation theory in $V$ is justified for large $N$ for the upper part of the spectrum, while several lowest energy eigenstates are affected strongly and have to be re-diagonalized. It turns out that the matrix elements $\left\langle\Psi_{q}|V| \Psi_{q}\right\rangle$ for low-lying energy eigenstates can be computed in terms of eigenvalues of the cyclic permutation operator

$$
\mathcal{P} \Psi_{q}\left(x_{1}, x_{2}, x_{3}\right)=\Psi_{q}\left(x_{3}, x_{1}, x_{2}\right)=e^{-i \phi_{q}} \Psi_{q}\left(x_{1}, x_{2}, x_{3}\right),
$$

where the phases $\phi_{q}$ take quantized values $\phi_{q}=0, \frac{2 \pi}{3}, \frac{4 \pi}{3}\left(\right.$ since $\left.\mathcal{P}^{3}=1\right)$. One obtains [18]

$$
\left\langle\Psi_{q^{\prime}}|V| \Psi_{q}\right\rangle=-\frac{\pi^{2}}{9} \frac{1}{\ln N} \cos \left(\phi_{q}-\phi_{q^{\prime}}\right)
$$

It is easy to see that the possible values of $\cos \left(\phi_{q} \pm \phi_{q^{\prime}}\right)$ are 1 and $-1 / 2$ depending on whether the phases $\phi_{q}$ and $\phi_{q^{\prime}}$ coincide. Introducing an integer $k=0, \pm 1, \pm 2, \ldots$ to enumerate quantized values of $q$ starting from the ones 
with the lowest absolute value, we get

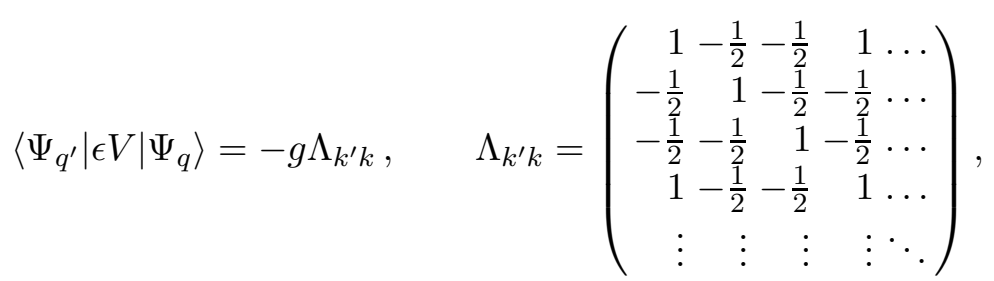

where the dependence on $\epsilon$ and $\eta$ is absorbed in the 'effective coupling' $g=\frac{\epsilon \pi^{2}}{9 \ln N}$. Notice the $\sim 1 / \ln N$ falloff for large $N$, which is slower than the level splitting for $\mathcal{H}_{3 / 2}, \delta E_{3 / 2} \sim 1 /(\ln N)^{2}$, confirming our expectation that $\sim \ln N$ lowest levels have to be re-diagonalized. Neglecting, for a moment, the splitting between unperturbed levels up to some number $k_{\max } \sim \ln N$, the true eigenstates would coincide with those of $\Lambda_{k k^{\prime}}$ (of the size $k_{\max }$ ), and the corresponding eigenvalues would define the energies. Remarkably, the spectrum of $\Lambda_{k k^{\prime}}$ is extremely simple. One can easily convince oneself that $\Lambda_{k k^{\prime}}$ has two and only two nonzero eigenvalues which are both equal to $k_{\text {max }}$. Remembering that $g \sim 1 / \ln N$ and $k_{\max } \sim \ln N$, this implies that two energy levels will get shifted by the finite amount $g k_{\max }=\mathcal{O}\left(\ln ^{0} N\right)$ while all the other ones remain exactly degenerate to this accuracy. Since (2.74) was derived up to corrections of order $\sim 1 / \ln ^{2} N$, this implies that the true energy shift for all levels apart from the lowest two is, at most, $\sim 1 / \ln ^{2} N$. This explains the creation of the mass gap observed in Fig. 3.

The size of the gap can be calculated for small $\epsilon$ by constructing an effective Hamiltonian for the low-lying eigenstates [18]. The idea of the solution is to interpret the integer $k$ as a discrete momentum variable. The corresponding wave functions in configuration space correspond to BlochFloquet waves and the resulting Schrödinger equation turns out to be a generalization of the famous Kroning-Penney model of a single particle in a periodic $\delta$-function potential. The result for the mass gap reads

$$
\Delta(\epsilon)=\mathcal{E}_{\text {bound }}-E_{0}=-\epsilon^{2} \frac{\pi^{4}}{72 \zeta(3)}+\mathcal{O}\left(\epsilon^{3}\right) .
$$

Extension of this approach to the physically interesting case $\epsilon \sim 1$ is nontrivial. However, the mass gap can easily be calculated in the opposite limit $\epsilon \gg 1$, and the result for $\epsilon=1$ found by matching the two expansions using Padé approximants: $\Delta_{1 / 2} \equiv \Delta(\epsilon=1)=-(0.32 \pm 0.02)$ [18].

Creation of the mass gap implies that the wave function of the ground state is drastically modified. For the integrable case, the eigenfunction corresponding to the lowest energy and hence $q=0$ can be found exactly. The 
physical interpretation of this result is most transparent in coordinate space. Up to contribution of operators with total derivatives, one can represent the 'ground state' of three quarks with the same helicity in a concise form as the nonlocal light-cone operator

$$
B_{3 / 2}^{(q=0)}\left(z_{1}, z_{2}, z_{3}\right)=\frac{1}{2} \sum_{\substack{a, b=1,2,3 \\ a \neq b}} \varepsilon^{i j k} \int_{0}^{1} d v \not h \psi_{i}^{\uparrow}\left(z_{a}\right) \not h \psi_{j}^{\uparrow}\left(v z_{a}+(1-v) z_{b}\right) \not h \psi_{k}^{\uparrow}\left(z_{b}\right) .
$$

The Taylor expansion of (2.76) at short distances, $z_{12}, z_{32} \rightarrow 0$, generates the series of local multiplicatively renormalizable three-quark operators with the lowest anomalous dimension for each even $N$. Note the integration in Eq. (2.76) with unit weight over the position of the quark in the middle that goes in between the light-cone positions of the other two quarks, up to permutations. If renormalization of the operator is interpreted as interaction, this unit weight can in turn be interpreted as the statement that the quark in the middle is effectively 'free': In the 'ground state' with the lowest "energy", the interaction of the quark in the middle with its right and left neighbors exactly compensate each other.

For the $B^{1 / 2}$, case the ground wave function changes dramatically and in the limit of large $N$ becomes, in the same sense as (2.76),

$$
\begin{aligned}
B^{ \pm}\left(\alpha_{1}, \alpha_{2}, \alpha_{3}\right)=\varepsilon^{i j k} & {\left[\left(\not h \psi_{i}^{\uparrow} \not h \psi_{j}^{\downarrow}\right)\left(\alpha_{1}\right) \not h \psi_{k}^{\uparrow}\left(\alpha_{3}\right) \delta\left(\alpha_{2}-\alpha_{1}\right)\right.} \\
& \left.\left. \pm \not h \psi_{i}^{\uparrow}\left(\alpha_{1}\right)\left(\not h \psi_{j}^{\downarrow}\right) \not h \psi_{k}^{\uparrow}\right)\left(\alpha_{3}\right) \delta\left(\alpha_{2}-\alpha_{3}\right)\right],
\end{aligned}
$$

where the two solutions correspond to states with different parity (cf. Fig. 33). Formation of the mass gap in the spectrum of anomalous dimensions is, therefore, naturally interpreted as due to binding of the quarks with opposite helicity into scalar diquarks.

Note that while the expression (2.76) for the eigenfunction is exact, the result (2.77) is only valid in the asymptotic $\ln N \rightarrow \infty$ limit. In the coordinate space picture, the restriction to large $N$ is translated to the condition that the light-cone separation between the same helicity quarks is very large to allow for the formation of a diquark. In other words, the diquark itself has a large size. In the momentum space, the result means that at sufficiently large normalization scale $\mu^{2}$ the quark carrying a very large momentum fraction is more often with the same helicity as of the parent baryon. This observation seems to be in qualitative agreement with phenomenological models of baryon distribution amplitudes. 


\subsection{Open spin chains}

There are other multiparticle operators which represent phenomenological interest and which were studied along the same line as the three-quark operators. For the twist-three sector, in addition to three-quark operators that we have addressed above one considers quark-antiquark-gluon and three-gluon operators. They arise in particular in connection with the structure function $g_{2}\left(x, Q^{2}\right)$ measured in polarized deep-inelastic scattering. This is attracting increasing interest as it provides a direct measurement of three-parton correlations in the proton. The scale-dependence of $g_{2}\left(x, Q^{2}\right)$ can be traced to the renormalization-group equations for the quark-antiquark-gluon operator [56]

$$
S^{ \pm}\left(z_{1}, z_{2}, z_{3}\right)=\frac{1}{2} \bar{\psi}\left(z_{1}\right)\left[i g \widetilde{F}_{\perp+}\left(z_{2}\right) \pm g F_{\perp+}\left(z_{2}\right) \gamma_{5}\right] \not h \psi\left(z_{3}\right),
$$

where $\widetilde{F}_{\mu \nu}=\frac{1}{2} \varepsilon_{\mu \nu \rho \lambda} F^{a, \rho \lambda} t^{a}, F_{\mu \nu}=F_{\mu \nu}^{a} t^{a}$. In the flavor singlet sector, one also has to include the three-gluon operator

$$
\widetilde{O}\left(z_{1}, z_{2}, z_{3}\right)=\frac{i}{2} g f^{a b c} F_{+\lambda}^{a}\left(z_{1}\right) \widetilde{F}_{+\perp}^{b}\left(z_{2}\right) F_{+\lambda}^{c}\left(z_{3}\right),
$$

which mixes with the C-even part of the flavor singlet quark-antiquark-gluon operator operator (2.78).

To begin with, consider the flavor non-singlet sector. In this case the three-gluon operator drops out and the evolution of $S^{ \pm}$simplifies dramatically in the limit of large number of colors, $N_{c} \rightarrow \infty$. The corresponding Hamiltonian reads, e.g., for $S^{+}[10,12]$

$$
\mathbb{H}_{S^{+}}=\frac{\alpha_{s}}{2 \pi} N_{c}\left[V_{q G}\left(J_{12}\right)+U_{G q}\left(J_{23}\right)\right]+\mathcal{O}\left(1 / N_{c}\right)
$$

where

$$
\begin{aligned}
V_{q G}(J) & =\psi\left(J+\frac{3}{2}\right)+\psi\left(J-\frac{3}{2}\right)-2 \psi(1)-\frac{3}{4}, \\
U_{G q}(J) & =\psi\left(J+\frac{1}{2}\right)+\psi\left(J-\frac{1}{2}\right)-2 \psi(1)-\frac{3}{4},
\end{aligned}
$$

and it turns out to be equivalent to the Hamiltonian of the completely integrable open Heisenberg magnet [10]. The corresponding conserved charge is equal to

$$
\mathcal{Q}_{S^{+}}=\left\{L_{12}^{2}, L_{23}^{2}\right\}-\frac{1}{2} L_{12}^{2}-\frac{9}{2} L_{23}^{2},
$$

where $\{$,$\} stands for an anticommutator. Properties of the Hamiltonian$ (2.80) were studied in detail in Refs. $[10,13,14,19]$ and the corrections $\mathcal{O}\left(1 / N_{c}^{2}\right)$ to the spectrum were calculated in Ref. [21]. The most interesting result is that the lowest energy, albeit the lowest anomalous dimension in 
the spectrum of quark-gluon operators can be exactly found, and the corresponding eigenfunction coincides to leading logarithmic accuracy with the structure function $g_{2}$ itself. Thus, a simple evolution equation arises [57].

The study of the flavor singlet sector is much more challenging [22]. Ignoring quarks for a moment, the evolution kernel for the three-gluon operators (2.79) can be mapped to the Hamiltonian of closed Heisenberg magnet with three "gluonic" sites of the spin $j_{g}=3 / 2$, whose integrability is "softly" broken by additional terms [13]. ${ }^{\mathrm{e}}$ This case was studied using the same techniques as the three-quark operators, although the algebra becomes more complicated because of the higher spins involved. The most interesting part appears to be the mixing between quark-gluon and three-gluon operators which for large $N$ can be interpreted as describing the interaction between open and closed Heisenberg magnets. It turns out that this mixing has rather peculiar features, which we cannot discuss in detail in this review, but the outcome is that the mixing can to a large extent be reduced to a few participating levels. Identifying important degrees of freedom (at least for large $N$ ) one can write down an approximate two-channel evolution equation for the structure function $g_{2}$ in terms of transverse spin densities [22].

\subsection{Quasipartonic and non-quasipartonic operators}

The operators we have discussed above all fall into the same class of the so-called quasipartonic operators, introduced in Ref. [12]. Since an offspring of this formalism will be useful in the discussion of supersymmetric extensions of $\mathrm{QCD}$, let us elaborate upon it. The approach heavily relies on the light-cone gauge, $A_{+}=0$. In this gauge, only physical degrees of freedom propagate, i.e., "good" components of the quark field $\psi_{+}$and circularly polarized gluons $\boldsymbol{A}_{\perp}^{\mu}$. Thus the identification of the elementary fields entering the Lagrangian with partonic states is straightforward - hence the name quasipartonic operators. Introducing the helicity operator as ${ }^{f}$

$$
h \equiv \overline{\boldsymbol{e}}_{\perp}^{i} \boldsymbol{e}_{\perp}^{j} \Sigma^{i j}, \quad \boldsymbol{e}_{\perp}^{i}=\left(\overline{\boldsymbol{e}}_{\perp}^{i}\right)^{*}=\frac{1}{\sqrt{2}}(1, i),
$$

one decomposes all fields in components with a fixed helicity. In particular, the left $\psi_{+}^{\downarrow}$ and right $\psi_{+}^{\uparrow}$ quarks, together with (anti)holomorphic components of the two-component propagating gauge potential $A \equiv \frac{1}{\sqrt{2}}\left(A^{x}+i A^{y}\right)$

e The exactly integrable chain arises for maximal-helicity gluonic operators [13].

${ }^{\mathrm{f}}$ Do not confuse helicity $h$ with the light-cone spin, discussed above. 
and $\bar{A} \equiv A^{*}$ are eigenfunctions of the helicity operator corresponding to

$$
\begin{array}{cc}
h \psi_{+}^{\uparrow}(x)=\frac{1}{2} \psi_{+}^{\uparrow}(x), & h \psi_{+}^{\downarrow}(x)=-\frac{1}{2} \psi_{+}^{\downarrow}(x), \\
h \bar{A}(x)=\bar{A}(x), & h A(x)=-A(x) .
\end{array}
$$

Let us notice in passing that the "good" quark fields of definite helicity have a single nonzero complex component, which suggests that one can entirely get rid of the spinor indices. A general quasipartonic operator is built from the above parton fields

$$
\mathbb{O}\left(z_{1}, \ldots\right)=\prod_{i} A\left(z_{i}\right) \prod_{j} \bar{A}\left(z_{j}\right) \prod_{k} \psi_{+}^{\uparrow}\left(z_{k}\right) \prod_{l} \psi_{+}^{\downarrow}\left(z_{l}\right) .
$$

Its covariant analogue can be found expressing the (anti)holomorphic gauge potentials in terms of the field strength tensor,

$$
\begin{aligned}
& A=\frac{1}{\sqrt{2}} \partial_{+}^{-1}\left(F^{+x}-i \widetilde{F}^{+x}\right)=\frac{i}{\sqrt{2}} \partial_{+}^{-1}\left(F^{+y}-i \widetilde{F}^{+y}\right), \\
& \bar{A}=\frac{1}{\sqrt{2}} \partial_{+}^{-1}\left(F^{+x}+i \widetilde{F}^{+x}\right)=-\frac{i}{\sqrt{2}} \partial_{+}^{-1}\left(F^{+y}+i \widetilde{F}^{+y}\right) .
\end{aligned}
$$

Contrary to "good" operators, the evolution of operators containing "bad" components of field operators does not have, in general, a simple pairwise pattern of the renormalization group mixing even at leading order in the coupling constant. A few exceptions do exist, however, for example in the case of the two-quark sector with Dirac structures

$$
\bar{\psi}\left(z_{1} n\right)\left(1, \gamma_{\perp}^{\mu}, \gamma_{\perp}^{\mu} \gamma^{5}\right) \psi\left(z_{2} n\right) .
$$

After separation of twist-two and three components from these operators, one finds that, in the large- $N_{c}$ limit, both of them evolve autonomously with different pairwise Hamiltonians. The twist-two part is well known. The eigenvalues of the twist-three Hamiltonian coincide with the lowest anomalous dimensions for quark-antiquark-gluon operators introduced in Sect. 2.6 ( see $[57-60])$.

The situation with more generic non-quasipartonic operators is an open issue. One of the major complications is that, in distinction with quasipartonic operators, the construction of the irreducible basis of non-quasipartonic operators is a nontrivial task. Up to now there exists no constructive procedure for the classification of non-quasipartonic operators of arbitrary high twist. The representation theory used in the analysis of usual quasipartonic operators is not very handy since it does not allow one to get rid of the redundancy of the operators in the basis. The redundancy in the basis, in 
turn, leads to complications in finding the eigensystem for the mixing matrix. Currently it can be solved only on a case-by-case basis. Finding a systematic procedure is an open question for further research.

A particular case of certain non-quasipartonic local multi-gluon higherdimension operators of the type

$$
\prod_{j=1}^{L} F_{\mu_{j} \nu_{j}}(0)
$$

in pure gluodynamics have been addressed recently and found to possess integrable structures corresponding to the compact spin-one Heisenberg magnet [61]. ${ }^{\mathrm{g}}$ Without loss of generality we can rephrase their analysis entirely in Euclidean space. The strength tensor can be decomposed into irreducible components

$$
F_{\mu \nu}=\eta_{\mu \nu}^{A} F_{+}^{A}+\bar{\eta}_{\mu \nu}^{A} F_{-}^{A}
$$

with the help of 't Hooft symbols, $O(4) \sim S U(2) \otimes S U(2)$. The selfdual and anti-selfdual components transform as $(1,0)$ and $(0,1)$, respectively. The part of the RG Hamiltonian responsible for eigenvalues of autonomous components does not change the number of fields in the local gluonic operator (2.88). By matching the coefficient of different irreducible components, extracted by means of projectors $\mathbb{P}_{\left(j_{1}, j_{2}\right)}^{P}$ for spin- $j$ and parity $P$, to the available one-loop calculations for gluonic operators up to dimension eight, the pairwise Hamiltonian was found to be [61]

$$
\mathbb{H}_{12}=7\left(\mathbb{P}_{(2,0)}+\mathbb{P}_{(0,2)}\right)+\mathbb{P}_{(1,0)}+\mathbb{P}_{(0,1)}-11\left(\mathbb{P}_{(0,0)}^{+}+\mathbb{P}_{(0,0)}^{-}\right)+3 \mathbb{P}_{(1,1)}^{-}
$$

The projection on the selfdual operators, i.e., built from products of $F_{+}^{A}$, reduces the above Hamiltonian to

$$
\mathbb{H}_{12}^{\text {sd }}=7 \mathbb{P}_{(2,0)}+\mathbb{P}_{(1,0)}-11 \mathbb{P}_{(0,0)}
$$

where the projection operators extract maximal-spin, antisymmetric and trace components and have the following obvious representation

$$
\begin{aligned}
& \mathbb{P}_{(2,0)} F_{+}^{A} F_{+}^{B}=\frac{1}{2}\left(F_{+}^{A} F_{+}^{B}+F_{+}^{A} F_{+}^{B}-\frac{2}{3} \delta^{A B} F_{+}^{C} F_{+}^{C}\right) \\
& \mathbb{P}_{(1,0)} F_{+}^{A} F_{+}^{B}=\frac{1}{2}\left(F_{+}^{A} F_{+}^{B}-F_{+}^{A} F_{+}^{B}\right) \\
& \mathbb{P}_{(2,0)} F_{+}^{A} F_{+}^{B}=\frac{1}{3} \delta^{A B} F_{+}^{C} F_{+}^{C} .
\end{aligned}
$$

g Other operators exist but do not affect the anomalous dimensions matrix as was demonstrated in [62] for $L=3,4$ by explicit calculation. 
They can be easily related to the permutation $P F_{+}^{A} F_{+}^{B}=F_{+}^{B} F_{+}^{A}$, trace $K F_{+}^{A} F_{+}^{B}=\delta^{A B} F_{+}^{C} F_{+}^{C}$ and identity $I F_{+}^{A} F_{+}^{B}=F_{+}^{A} F_{+}^{B}$ operators,

$$
\mathbb{P}_{(2,0)}=\frac{1}{2}(I+P)-\frac{1}{3} K, \quad \mathbb{P}_{(1,0)}=\frac{1}{2}(I-P), \quad \mathbb{P}_{(0,0)}=\frac{1}{3} K .
$$

Then, the pairwise Hamiltonian can be brought to the form

$$
\mathbb{H}_{12}^{\mathrm{sd}}=4 I_{12}+3 P_{12}-6 K_{12}=7+3 s_{1} \cdot s_{2}\left(1-s_{1} \cdot s_{2}\right)
$$

where in the second equality we have used the representation in terms of spin-one $S U(2)$ generators. This is a Hamiltonian of an exactly solvable spinone Heisenberg magnet which can be diagonalized by means of the Bethe Ansatz $[3,4,63]$. It is interesting that the ground state of the corresponding spin-one chain is antiferromagnetic contrary to the ferromagnetic situation for the spin chains governing the renormalization of the scalar operators in $\mathcal{N}=4$ SYM theory.

\subsection{Conformal symmetry breaking}

Nothing is known at present about whether integrable structures survive in QCD beyond leading logarithms, in which case conformal symmetry is broken by the trace anomaly of the energy-momentum tensor. The constraints imposed by conformal symmetry, and, in particular, by conformal Ward identities, appear to be nontrivial and can be used to advance perturbative calculations to higher orders. The idea has been to establish the following schematic structure of the perturbative series for a generic quantity $\mathcal{Q}$

$$
\mathcal{Q}=\mathcal{Q}^{\text {con }}+\frac{\beta(g)}{g} \Delta \mathcal{Q}, \quad \text { where } \Delta \mathcal{Q}=\text { power series in } \alpha_{s} .
$$

Here $\mathcal{Q}^{\text {con }}$ is the result in the formal conformal limit, obtained by setting the $\beta$-function to zero by hand. It has the full symmetry of a conformallyinvariant theory. The extra term $\Delta \mathcal{Q}$ can be perturbatively evaluated as a power series in $\alpha_{s}$ and vanishes to leading order (LO). Note that the evaluation of the leading $\mathcal{O}\left(\alpha_{s}\right)$ contribution to $\Delta \mathcal{Q}$ requires little effort, since it is sufficient to calculate the $N_{f}$ proportional terms in $\beta_{0}=11 N_{c} / 3-2 N_{f} / 3$ via quark bubble insertions.

The possibility of the separation of the conformally-symmetric and $\beta$ proportional contributions is by no means trivial. The reason is that although the dilatation anomaly to $\mathcal{O}\left(\alpha_{s}\right)$ accuracy receives contributions only from UV-divergent parts of relevant Feynman diagrams and is diagonal for conformal operators, the special conformal anomaly (to the same accuracy) 
is affected by the finite parts of the same (one-loop) diagrams and is not diagonal in a general, MS-like, renormalization scheme [64]. The nondiagonal entries in the special conformal anomaly can, however, be removed and the special conformal covariance (neglecting the $\beta$-function) restored by performing a finite renormalization [64]. This transformation defines the conformal subtraction scheme in which the separation in (2.93) becomes meaningful. Whether the remnants of integrability survive in this procedure, remains to be seen.

\section{Dilatation operators in supersymmetric gauge theories}

During the last year, a lot of new and interesting results have been obtained on the integrability properties of the $\mathcal{N}=4 \mathrm{SYM}$ theory. These studies are important and provide hints on the long standing quest to find the correspondence between (supersymmetric) Yang-Mills theories at strong coupling and noncritical strings propagating on the curved background. Our aim in this section is to establish a connection between these new developments and the integrability of RG equations in QCD discussed in the previous section. In particular, the key question is to distinguish between phenomena that are already present in pure Yang-Mills theory (and only get enhanced by the supersymmetry), and ones that present genuinely new features of SYM gauge theories.

In order to trace this connection, we introduce a superfield formalism in which all symmetries of the theory become manifest and calculations can be performed in a unified manner for different numbers of supercharges $\mathcal{N}=0,1,2,4$. The maximally-supersymmetric $\mathcal{N}=4 \mathrm{SYM}$ theory is a finite, four-dimensional conformal field theory $[45,46,65,66]$, while the $\mathcal{N}=$ 0 theory corresponds to pure gluodynamics. Unfortunately, no covariant superfield formulation for $\mathcal{N}=4$ exists, which makes this case somewhat special. Because of this, we employ a noncovariant, light-cone superspace formalism due to Mandelstam [45] and Brink et al. [46]. This technique was used, e.g., to prove the finiteness of the $\mathcal{N}=4 \mathrm{SYM}$ theory but is not very popular nowadays. Hence we begin this section by reviewing this beautiful framework.

We will find it useful to introduce multi-particle single trace operators built from light-cone superfields at "super-light-cone" separations in the superspace $Z_{k}=\left(z_{k}, \theta_{k}^{A}\right)$ where $z_{k}$ are the usual "even" light-cone coordinates and $\theta_{k}^{A}$ are a set of Grassmanian "odd" coordinates. The one-loop dilatation operator for such operators can be found in terms of simple field displacements on the super-light-cone, similarly to the QCD case. Expanding such 
operators in "even" and "odd" coordinates, we will be able to reproduce the integrability properties of QCD operators with multiple derivatives discussed in the previous section [10], on one side, and composite scalar operators, considered in [25] on the other side, which are related to $S L(2, \mathbb{R})$ and $S O(6)$ spin chains, respectively. Hence, these two results, that were found independently, appear to be just two sides of the same coin. We will then comment on the extension of these results to other operators.

\subsection{SYM theories in light-cone superspace}

All super-Yang-Mills theories in four dimensions can be obtained through dimensional reduction from a higher-dimensional $\mathcal{N}=1$ gauge theory [67]

$$
S=\int d^{D} x\left\{-\frac{1}{4} F_{M N}^{a} F_{a}^{M N}+\frac{i}{2} \bar{\psi}^{a} \Gamma_{M}\left(\partial^{M} \delta_{a b}+g f^{a c b} A_{c}^{M}\right) \psi^{b}\right\}
$$

describing the interaction of the $D$-dimensional gauge field $A^{M}$ with the $2^{[D / 2]}$-component Majorana-Weyl fermion $\psi$, both belonging to the adjoint representation of the gauge $S U\left(N_{c}\right)$ group. In particular, the $\mathcal{N}=2 \mathrm{SYM}$ theory can be obtained from the $D=6$ action,

$$
\begin{aligned}
\mathcal{L}_{\mathcal{N}=2}=\operatorname{tr} & \left\{-\frac{1}{2} F_{\mu \nu} F^{\mu \nu}+2 i \bar{\lambda}_{\dot{\alpha} A} \sigma_{\mu}^{\dot{\alpha} \beta} \mathcal{D}^{\mu} \lambda_{\beta}^{A}+2\left(\mathcal{D}_{\mu} \bar{\phi}\right)\left(\mathcal{D}^{\mu} \phi\right)\right. \\
& \left.-g^{2}[\phi, \bar{\phi}]^{2}-\sqrt{2} g \varepsilon_{A B} \lambda^{\alpha A}\left[\bar{\phi}, \lambda_{\alpha}^{B}\right]+\sqrt{2} g \varepsilon^{A B} \bar{\lambda}_{\dot{\alpha} A}\left[\phi, \bar{\lambda}_{B}^{\dot{\alpha}}\right]\right\}
\end{aligned}
$$

(with $A, B=1,2$ ), and the $\mathcal{N}=4$ SYM theory follows from from $D=10$ action,

$$
\begin{aligned}
\mathcal{L}_{\mathcal{N}=4}= & \operatorname{tr}\left\{-\frac{1}{2} F_{\mu \nu} F^{\mu \nu}+\frac{1}{2}\left(\mathcal{D}_{\mu} \phi^{A B}\right)\left(\mathcal{D}^{\mu} \bar{\phi}_{A B}\right)+\frac{1}{8} g^{2}\left[\phi^{A B}, \phi^{C D}\right]\left[\bar{\phi}_{A B}, \bar{\phi}_{C D}\right]\right. \\
& \left.+2 i \bar{\lambda}_{\dot{\alpha} A} \sigma_{\mu}^{\dot{\alpha} \beta} \mathcal{D}^{\mu} \lambda_{\beta}^{A}-\sqrt{2} g \lambda^{\alpha A}\left[\bar{\phi}_{A B}, \lambda_{\alpha}^{B}\right]+\sqrt{2} g \bar{\lambda}_{\dot{\alpha} A}\left[\phi^{A B}, \bar{\lambda}_{B}^{\dot{\alpha}}\right]\right\},
\end{aligned}
$$

(with $A, B=1, \ldots, 4$ ). Obviously, the first term on the r.h.s. of Eq. (3.2) gives the Lagrangian of a pure gauge, $\mathcal{N}=0$, theory, while the sum of the first two terms defines (for $A=1$ ) the $\mathcal{N}=1$ SYM theory in the Weyl form. Thus, augmentation of supersymmetry increases the number of fundamental fields in the theory, which has profound consequences. The $\mathcal{N}=0,1 \mathrm{SYM}$ theories possess a non-vanishing beta-function which receives contributions at all orders of the perturbative expansion in the coupling. In $\mathcal{N}=2$ theory the beta-function gets a contribution at one-loop only, while for $\mathcal{N}=4$ 
the beta function vanishes to all orders. Hence the $\mathcal{N}=4$ theory remains superconformally-invariant at the quantum level $[45,46,65,66]$.

In order to construct a SYM theory on the light-cone, one starts with the component form of the action and fixes the light-cone gauge $A_{+}(x)=0$. In this gauge, one sacrifices four-dimensional Lorentz covariance in favor of a unified description of $\mathcal{N}$-extended SYM theories in superspace. The main steps in designing this formalism $[45,46,68]$ consist of (i) making use of the decomposition of the elementary fields into propagating and non-propagating modes in light-cone time $x_{+}{ }^{\mathrm{h}}$, in the same way as indicated above in the context of QCD, (ii) integrating non-dynamical fields out in the functional integral, (iii) assembling the propagating fields into superfields, and finally (iv) casting the light-cone component action into the superspace form.

The first two steps can be done for a generic $D$-dimensional $\mathcal{N}=1$ Lagrangian without specifying the value of $D$. One finds that in the light-cone gauge $A_{+}(x)=0$, the action (3.1) involves two non-propagating fields $[45,46,68]$ : one bosonic $S^{a}$ and one fermionic $\chi^{a}$

$$
\begin{aligned}
S^{a} & =\partial_{+} A_{-}^{a}-\partial_{+}^{-1} \mathcal{D}_{i}^{a b} \partial_{+} \boldsymbol{A}_{i}^{b}-\frac{i}{2} g f^{b a c} \partial_{+}^{-1} \bar{\psi}_{+}^{b} \Gamma_{+} \psi_{+}^{c}, \\
\chi^{a} & =\psi_{-}^{a}-\frac{1}{2} \partial_{+}^{-1} \Gamma_{+} \Gamma^{i} \mathcal{D}_{i}^{a b} \psi_{+}^{b},
\end{aligned}
$$

which can be set to zero. Here $\mathcal{D}_{a b}^{i}=-\delta_{a b} \partial_{\boldsymbol{x}}^{i}+g f_{a c b} \boldsymbol{A}_{c}^{i}$ is the transverse covariant derivative. Solving $S^{a}=\chi^{a}=0$, one obtains the expressions for $A_{-}^{a}$ and $\psi_{-}^{a}$ in terms of the propagating fields and substitutes them back into the SYM action. As the next step, one performs the reduction of the resulting action to four dimensions, giving rise to various SYM theories on the light-cone. Their explicit form for $\mathcal{N}>1$ is very lengthy and is not of interest per se, but rather as an intermediate result on the way to achieving the superspace form.

We go directly to step (iii) and decompose all propagating, "physical" fields into definite helicity components. In the case of $\mathcal{N}=4 \mathrm{SYM}$, ${ }^{\mathrm{i}}$ they include (anti)holomorphic, helicity- \pm 1 fields, $A=\left(A_{1}+i A_{2}\right) / \sqrt{2}$ and $\bar{A}=\left(A_{1}-i A_{2}\right) / \sqrt{2}$, built from two-dimensional transverse components of the gauge field, $A_{\perp}(x)=\left(A_{1}, A_{2}\right)$, complex scalar fields $\phi^{A B}$ of helicity 0 and helicity $\pm 1 / 2$ components of Majorana-Weyl fermions, $\lambda_{\alpha}^{A}$ and $\bar{\lambda}_{A}^{\dot{\alpha}}$. An important property of the light-cone formalism, which makes it advantageous over the covariant one, is that the latter fields have only one non-vanishing

${ }^{\mathrm{h}}$ I.e., those that have or do not have the kinetic term with light-cone time derivative $\partial_{-}$.

${ }^{i}$ As we will see in a moment, the SYM light-cone theories with less supersymmetry can be obtained from the $\mathcal{N}=4$ theory through the truncation procedure. 
component $\lambda_{1}^{A} \equiv \sqrt[4]{2} \lambda^{A}$ and $\bar{\lambda}_{A}^{2} \equiv i \sqrt[4]{2} \bar{\lambda}_{A}$. As a consequence, one can describe helicity- $\pm 1 / 2$ fermions by Grassmann-valued complex fields without any Lorentz index. Introducing the fermionic coordinates $\theta^{A}$ possessing the helicity $h \theta^{A}=-\frac{1}{2} \theta^{A}$ and their conjugates $\bar{\theta}_{A}$ with $h \bar{\theta}_{A}=\frac{1}{2} \bar{\theta}_{A}$, we can assemble the above fields into a single, complex, $\mathcal{N}=4$ superfield [46]

$$
\begin{aligned}
\Phi\left(x, \theta^{A}, \bar{\theta}_{A}\right) & =\mathrm{e}^{\frac{1}{2} \bar{\theta}_{A} \theta^{A} \partial_{+}}\left\{\partial_{+}^{-1} A(x)+\theta^{A} \partial_{+}^{-1} \bar{\lambda}_{A}(x)+\frac{i}{2 !} \theta^{A} \theta^{B} \bar{\phi}_{A B}(x)\right. \\
& \left.-\frac{1}{3 !} \varepsilon_{A B C D} \theta^{A} \theta^{B} \theta^{C} \lambda^{D}(x)-\frac{1}{4 !} \varepsilon_{A B C D} \theta^{A} \theta^{B} \theta^{C} \theta^{D} \partial_{+} \bar{A}(x)\right\},
\end{aligned}
$$

which satisfies the chirality condition

$$
\bar{D}^{B} \Phi\left(x, \theta^{A}, \bar{\theta}_{A}\right)=\left(\partial_{\bar{\theta}_{B}}-\frac{1}{2} \theta^{B} \partial_{+}\right) \Phi\left(x, \theta^{A}, \bar{\theta}_{A}\right)=0 .
$$

It embraces all particle helicities, from -1 to 1 with half-integer step, and, therefore, $\Phi\left(x, \theta^{A}, \bar{\theta}_{A}\right)$ describes a CPT-self-conjugate supermultiplet. Another advantage of the light-cone formalism is that the full supersymmetry algebra is reduced on the light-cone to a subalgebra which acts linearly on the "physical" fields and closes off-shell. In particular, in the $\mathcal{N}=4 \mathrm{SYM}$ theory, the supersymmetric transformations on the light-cone read as

$$
\begin{aligned}
& \delta A=\xi^{A} \bar{\lambda}_{A}, \\
& \delta \lambda^{A}=-\left(\partial^{+} \bar{A}\right) \xi^{A}-i\left(\partial^{+} \phi^{A B}\right) \bar{\xi}_{B}, \\
& \delta \phi^{A B}=i\left(\xi^{[A} \lambda^{B]}-\varepsilon^{A B C D} \bar{\xi}_{C} \bar{\lambda}_{D}\right),
\end{aligned}
$$

where $[A, B] \equiv A B-B A$.

Gauge theories on the light-cone with less or no supersymmetry can be deduced from the maximally supersymmetric $\mathcal{N}=4$ theory by removing "unwanted" physical fields. In the superfield formulation this amounts to a truncation of the $\mathcal{N}=4$ superfield, or equivalently, reduction of the number of fermionic directions in the superspace $[69,70]$. For instance, the $\mathcal{N}=2$ chiral superfield is

$$
\Phi^{(2)}=\left.\Phi^{(4)}\right|_{\theta^{3}=\theta^{4}=0}=\mathrm{e}^{\frac{1}{2} \bar{\theta}_{A} \theta^{A} \partial_{+}}\left\{\partial_{+}^{-1} A(x)+\theta^{A} \partial_{+}^{-1} \bar{\lambda}_{A}(x)+i \theta^{1} \theta^{2} \bar{\phi}(x)\right\}
$$

with the identification $\phi^{A B}=\varepsilon^{A B} \phi$ and $A, B=1,2$. To get the $\mathcal{N}=1$ superfields one removes three odd coordinates $\theta^{2}=\theta^{3}=\theta^{4}=0$, and, finally for $\mathcal{N}=0$ all $\theta$ 's in (3.5) have to be set to zero. Notice that under this procedure the truncated $\mathcal{N}=2, \mathcal{N}=1$ and $\mathcal{N}=0$ theories involve only half of the fields described by the $\mathcal{N}$-extended SYM theory and the other half of 
the needed particle content arises from the complex conjugated superfields $\bar{\Phi} \equiv \Phi^{*}$. For $\mathcal{N}=4$ the two superfields $\Phi$ and $\bar{\Phi}$ are related to each other through the reality condition $[45,46]$

$$
\Phi\left(x, \theta^{A}, \bar{\theta}_{A}\right)=-\partial_{+}^{-2} \bar{D}^{1} \bar{D}^{2} \bar{D}^{3} \bar{D}^{4} \bar{\Phi}\left(x, \theta^{A}, \bar{\theta}_{A}\right),
$$

but they are independent for $\mathcal{N} \leq 2$.

The final step consists of constructing the action for the SYM theory in terms of the light-cone superfields. One starts with the $\mathcal{N}=4$ theory, which involves a single superfield (3.5). The action consists of the kinetic term $\Phi \Phi$ as well as the cubic $\Phi \Phi \Phi$ and quartic couplings $\Phi \Phi \Phi \Phi$. Since light-cone supersymmetry is realized linearly, each of the above three terms is separately invariant under the supersymmetric transformations (3.7). Thus one can individually construct superspace invariants for each number of superfields and match them with the light-cone action in the component form to fix the overall normalization. This yields [46]

$$
\begin{aligned}
S_{\mathcal{N}=4} & =\int d^{4} x d^{4} \theta d^{4} \bar{\theta}\left\{\frac{1}{2} \bar{\Phi}^{a} \square \partial_{+}^{-2} \Phi^{a}-\frac{2}{3} g f^{a b c}\left(\partial_{+}^{-1} \bar{\Phi}^{a} \Phi^{b} \bar{\partial} \Phi^{c}+\partial_{+}^{-1} \Phi^{a} \bar{\Phi}^{b} \partial \bar{\Phi}^{c}\right)\right. \\
& \left.-\frac{1}{2} g^{2} f^{a b c} f^{a d e}\left(\partial_{+}^{-1}\left(\Phi^{b} \partial_{+} \Phi^{c}\right) \partial_{+}^{-1}\left(\bar{\Phi}^{d} \partial_{+} \bar{\Phi}^{e}\right)+\frac{1}{2} \Phi^{b} \bar{\Phi}^{c} \Phi^{d} \bar{\Phi}^{e}\right)\right\}, \quad(3.10)
\end{aligned}
$$

with $\bar{\Phi}$ given by (3.9). The SYM theories with less supersymmetry, $\mathcal{N} \leq 2$, can be derived from (3.10) through the truncation procedure. For $\mathcal{N}=0,1,2$ the resulting light-cone action has the universal form [69]

$$
\begin{aligned}
S_{\mathcal{N} \leq 2} & =\int d^{4} x d^{\mathcal{N}} \theta d^{\mathcal{N}} \bar{\theta}\left\{\sigma_{\mathcal{N}} \bar{\Phi}^{a} \square \partial_{+}^{2-\mathcal{N}} \Phi^{a}\right. \\
& -2 g f^{a b c} \sigma_{\mathcal{N}}\left(\partial_{+} \Phi^{a} \partial_{+}^{2-\mathcal{N}} \bar{\Phi}^{b} \bar{\partial} \Phi^{c}+(-1)^{\mathcal{N}} \partial_{+} \bar{\Phi}^{a} \partial_{+}^{2-\mathcal{N}} \Phi^{b} \partial \bar{\Phi}^{c}\right) \\
& \left.-(-1)^{\mathcal{N}} 2 g^{2} f^{a b c} f^{a d e} \partial_{+}^{-1}\left(\partial_{+} \Phi^{b} \overline{\boldsymbol{D}}^{\mathcal{N}} \partial_{+}^{2-\mathcal{N}} \bar{\Phi}^{c}\right) \partial_{+}^{-1}\left(\partial_{+} \bar{\Phi}^{d} \boldsymbol{D}_{\mathcal{N}} \partial_{+}^{2-\mathcal{N}} \Phi^{e}\right)\right\}
\end{aligned}
$$

where $\sigma_{\mathcal{N}}=(-1)^{\frac{\mathcal{N}(\mathcal{N}+1)}{2}}, \boldsymbol{D}_{\mathcal{N}} \equiv D_{1} \ldots D_{\mathcal{N}}$ and analogously for $\overline{\boldsymbol{D}}^{\mathcal{N}}$. Here, in contradistinction with Eq. (3.10), the superfields $\Phi$ and $\bar{\Phi}$ are independent on each other.

\subsection{Superconformal symmetry on the light-cone}

Our main objects of interest will be multiparticle single-trace operators built from light-cone superfields

$$
\mathbb{O}\left(Z_{1}, \ldots, Z_{L}\right)=\operatorname{tr}\left\{\Phi\left(Z_{1}\right) \Phi\left(Z_{2}\right) \cdots \Phi\left(Z_{L}\right)\right\},
$$


where $\Phi(Z) \equiv \Phi^{a}(Z) t^{a}$ is a matrix valued superfield in the fundamental representation of the gauge group and $Z$ denotes its position in the superspace with four even coordinates, $x_{\mu}$, and $2 \mathcal{N}$ odd coordinates, $\theta^{A}$ and $\bar{\theta}_{A}$ with $A=1, \ldots, \mathcal{N}$. In addition, we choose all superfields to be located along the light-cone direction in the four-dimensional Minkowski space defined by the light-like vector $n_{\mu}$ (with $n^{2}=0$ ) defined as in Eq. (2.1), so that $n \cdot A=A_{+}=0$, cf. (2.2). Similarly to the QCD case, the positions of the superfields on the light-cone are parameterized by real numbers $x_{\mu}=z n_{\mu}$. One can further simplify the notations by taking into account that a $\bar{\theta}$-dependence of the chiral superfields can be absorbed into the redefinition of $z$-variables

$$
\Phi\left(z n_{\mu}, \theta^{A}, \bar{\theta}_{A}\right)=\Phi\left(\left(z+\frac{1}{2} \theta \cdot \bar{\theta}\right) n_{\mu}, \theta^{A}, 0\right) .
$$

Redefining $z+\frac{1}{2} \theta \cdot \bar{\theta} \rightarrow z$, we shall specify the position of the superfield in the superspace as $Z_{k}=\left(z_{k}, \theta_{k}^{A}\right)$ so that $\Phi\left(Z_{k}\right) \equiv \Phi\left(z_{k} n_{\mu}, \theta_{k}^{A}, 0\right)$.

The single-trace operators (3.12) represent a natural generalization of nonlocal light-ray operators in QCD, cf. Eq. (2.6). To obtain the latter it is sufficient to expand $\mathbb{O}\left(Z_{1}, \ldots, Z_{L}\right)$ in powers of odd variables $\theta_{1}^{A_{1}} \ldots \theta_{L}^{A_{L}}$. As in QCD, nonlocal operators (3.12) serve as generating functions for Wilson operators with the maximal Lorentz spin and minimal twist equal to the number of constituent fields $L$. A question arises as to whether this set of Wilson operators is complete. In $\mathcal{N}=4$ SYM theory there is only one independent chiral superfield $\Phi(Z)$ and, as a consequence, the operators (3.12) generate all Wilson operators of twist $-L$ built from $L$ fundamental fields. For $\mathcal{N} \leq 2$, the superfields $\Phi(Z)$ and $\bar{\Phi}(Z)$ are independent of each other and, in addition to the operators in (3.12), one can introduce "mixed" operators built from both superfields. This means that in the $\mathcal{N}=0,1$ and 2 SYM theories, the operators (3.12) only generate a certain subset of the existing Wilson operators. The reason why we will concentrate on this subset is that it inherits integrability properties found in QCD in the sector of maximal helicity operators. Namely, as we shall argue below in this section, the one-loop dilatation operator acting on the space of single-trace operators (3.12) can be mapped in the multicolor limit into a Hamiltonian of a completely integrable Heisenberg spin magnet with the symmetry group being a collinear $S L(2 \mid \mathcal{N})$ subgroup of the full superconformal group [24].

To explain this correspondence, we would like to recall the symmetries of the SYM theories. At the classical level, a four-dimensional supersymmetric Yang-Mills theory with $\mathcal{N}$ supercharges is invariant under the $S U(2,2 \mid \mathcal{N})$ superconformal transformations [67]. The corresponding algebra contains 
both bosonic (even) and fermionic (odd) generators. The even generators are the fifteen generators of the $S O(2,4)$ conformal algebra familiar from QCD plus the chiral $\mathbf{R}$ charge and the $S U(\mathcal{N})$ internal "flavor" symmetry generators $\mathbf{T}_{A}^{B}$ (for $\mathcal{N} \geq 2$ only). The odd generators are the generators of supersymmetric and superconformal transformations, $\mathbf{Q}_{\alpha A}, \overline{\mathbf{Q}}^{\dot{\alpha} A}$ and $\mathbf{S}_{\alpha}^{A}, \overline{\mathbf{S}}_{A}^{\dot{\alpha}}$, respectively. Since the superfields entering (3.12) "live" on the light-cone, we expect, by analogy with the QCD case, that the full superconformal group will be reduced to its collinear subgroup. We know from Sect. 2.1 that in Yang-Mills theories with no supersymmetry, the collinear subgroup is generated by three bosonic operators $\mathbf{L}^{ \pm, 0}$ which form the $S L(2, \mathbb{R})$ algebra. Supersymmetry enlarges the $S L(2, \mathbb{R})$ subgroup by adding the following generators: the chiral charge $\mathbf{R}, S U(\mathcal{N})$ charges $\mathbf{T}_{A}{ }^{B}$, transverse boosts $\mathbf{M}_{12}$ and, finally, the "good" components of the fermionic charges $\mathbf{Q}_{A}, \overline{\mathbf{Q}}^{A}$, $\mathbf{S}^{A}$ and $\overline{\mathbf{S}}_{A}$. Being combined together, these operators form the $S L(2 \mid \mathcal{N})$ algebra [24].

To one-loop accuracy, the generators of the $S L(2 \mid \mathcal{N})$ subgroup can be realized as linear differential operators, $[\mathbf{G}, \Phi]=G\left(\partial_{z}, \partial_{\theta}\right) \Phi$, acting on the light-cone coordinates of the chiral superfield $\Phi(Z)[24,71]$. The corresponding expressions for the generators of the $S L(2 ; \mathbb{R})$ collinear subgroup generalize similar expressions in the QCD case, Eq. (2.18),

$$
L^{-}=-\partial_{z}, \quad L^{+}=2 j z+z^{2} \partial_{z}+z\left(\theta \cdot \partial_{\theta}\right), \quad L^{0}=j+z \partial_{z}+\frac{1}{2}\left(\theta \cdot \partial_{\theta}\right),
$$

and the twist operator is $E=t$. The four odd generators can be represented by the following differential operators

$$
W^{A,-}=\theta^{A} \partial_{z}, \quad W^{A,+}=\theta^{A}\left[2 j+z \partial_{z}+\left(\theta \cdot \partial_{\theta}\right)\right], \quad V_{A}^{-}=\partial_{\theta^{A}}, \quad V_{A}^{+}=z \partial_{\theta^{A}} .
$$

Finally, the $S U(\mathcal{N})$ generators and linear combination of the abelian $R$-charge and transverse boosts $\mathbf{M}_{12}$ are realized as

$$
T_{B}{ }^{A}=\theta^{A} \partial_{\theta^{B}}-\frac{1}{\mathcal{N}} \delta_{B}^{A}\left(\theta \cdot \partial_{\theta}\right), \quad B=-j-\frac{1}{2}\left(1-\frac{2}{\mathcal{N}}\right)\left(\theta \cdot \partial_{\theta}\right) .
$$

In these expressions, $\partial_{z} \equiv \partial / \partial z$ and $\theta \cdot \partial_{\theta} \equiv \theta^{A} \partial / \partial \theta^{A}$. The twist $t$ and conformal spin $j$ are the parameters defining the representation. For the chiral superfield $\Phi(Z)$ entering the Lagrangian of $\mathcal{N}$-extended SYM theories, Eqs. (3.10) and (3.12), one has $j=-\frac{1}{2}$ and $t=1$.

The light-cone operator $\mathbb{O}\left(Z_{1}, \ldots, Z_{L}\right)$, Eq. (3.12), serves as the generating function of an infinite tower of Wilson operators with an arbitrary number of derivatives, when expanded in the bosonic separations, and of different field content, when expanded in fermionic coordinates. The most efficient 
way to renormalize an infinite set of local Wilson operators is to study the renormalization properties of the light-ray operators $\mathbb{O}\left(Z_{1}, \ldots, Z_{L}\right)$ themselves. Superconformal invariance imposes severe restrictions on the possible form of the dilatation operator in the $\mathcal{N}$-extended SYM theory acting on the space of light-cone operators (3.12). For the operator $\mathbb{O}\left(Z_{1}, \ldots, Z_{L}\right)$, the generators of the $S L(2 \mid \mathcal{N})$ collinear subgroup are given by the sum of single-particle generators, Eqs. (3.14) - (3.16), acting on the coordinates in the superspace $Z_{k}=\left(z_{k}, \theta_{k}^{A}\right)$ with $k=1, \ldots, L$. In particular, the total twist of the operator $\mathbb{O}\left(Z_{1}, \ldots, Z_{L}\right)$ is equal to the number of superfields $L$. It is well known from QCD [12] that to one-loop accuracy the RG evolution of local quasipartonic operators preserves the number of particles. In addition, the mixing of single trace operators with operators involving larger number of traces is suppressed by powers of $1 / N_{c}^{2}$. Taken together, these two observations imply that to one-loop accuracy the set of light-cone operators $\mathbb{O}\left(Z_{1}, \ldots, Z_{L}\right)$ is closed under renormalization in the multicolor limit. The corresponding one-loop dilatation operator can be represented in the lightcone superfield formalism as a quantum mechanical Hamiltonian $\mathbb{H}$ leading to the following $\mathrm{RG}$ equation

$$
\left\{\mu \frac{\partial}{\partial \mu}+\beta(g) \frac{\partial}{\partial g}+L \gamma(g)\right\} \mathbb{O}^{\mathrm{R}}\left(Z_{1}, \ldots, Z_{L}\right)=-\frac{g^{2} N_{c}}{8 \pi^{2}}\left[\mathbb{H} \cdot \mathbb{O}^{\mathrm{R}}\right]\left(Z_{1}, \ldots, Z_{L}\right) .
$$

The renormalized operator $\mathbb{O}^{\mathrm{R}}$ generates finite Green functions and is expressed in terms of the bare operator (3.12) with an infinite multiplicative counterterm. Here $\gamma(g)$ is the anomalous dimension of the superfields in the light-like gauge $A_{+}(x)=0$. Thanks to the Ward identity it is related to the beta-function of the theory $\gamma(g)=\beta(g) / g$. The Hamiltonian $\mathbb{H}$ in Eq. (3.17) is an integral operator acting on the coordinates of the superfields in the superspace. Superconformal invariance implies that the dilatation operator $\mathbb{H}$ commutes with the generators of the $S L(2 \mid \mathcal{N})$ supergroup in Eqs. (3.14) (3.16).

\subsection{One-loop dilatation operator}

To one-loop order, the dilatation operator $\mathbb{H}$ has a pairwise structure. In addition, only the nearest-neighbor interactions survive in the multicolor limit, leading to

$$
\mathbb{H}=\mathbb{H}_{12}+\mathbb{H}_{23}+\cdots+\mathbb{H}_{L 1} .
$$

The two-particle interaction kernel $\mathbb{H}_{k, k+1}$ receives contributions from the Feynman diagrams shown in Fig. 4 They involve cubic and quartic inter- 

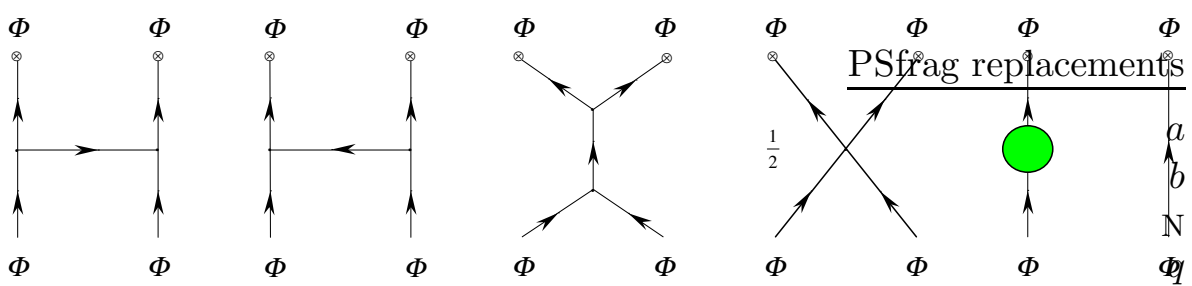

Figure 4. Feynman diagrams contributing to the one-loop dilatation operator for the two chiral superfields on the light-cone.

action vertices which are uniquely defined by the light-cone actions (3.10) and (3.12). As a consequence of superconformal symmetry the kernel $\mathbb{H}_{k, k+1}$ has to commute with the generators of the $S L(2 \mid \mathcal{N})$ superalgebra acting on the superfields with labels $k$ and $k+1$ and, therefore, it is a function of the two-particle quadratic Casimir operator

$$
\mathbb{H}_{k, k+1}=H\left(L_{k, k+1}^{2}\right),
$$

where a general expression for the quadratic Casimir in the $S L(2 \mid \mathcal{N})$ algebra is $[72]$

$$
\begin{aligned}
L^{2} & =\left(L^{0}\right)^{2}+L^{+} L^{-} \\
& +(\mathcal{N}-1) L^{0}+\frac{\mathcal{N}}{\mathcal{N}-2} B^{2}-V_{A}^{+} W^{A,-}-W_{A}^{+} V^{A,-}-\frac{1}{2} T^{B}{ }_{A} T^{A}{ }_{B} .
\end{aligned}
$$

For $\mathcal{N}=0$, this expression coincides with Eq. (2.21). In that case, the $S L(2)$ invariance allows one to obtain the general form of the two-particle kernels in QCD, Eq. (2.30).

We remind the reader that in the light-cone superspace formalism, the Yang-Mills theory without supersymmetry can be obtained from the one with $\mathcal{N}$ supercharges by putting the odd coordinates to zero, $\theta^{A}=0$. For this reason the integral operator (2.30) can be considered as the special limit of a general two-particle kernel $\mathbb{H}_{k, k+1}$ for $\theta_{k}^{A}=\theta_{k+1}^{A}=0$. Making use of the odd generators in the $S L(2 \mid \mathcal{N})$ algebra one can "lift" the operator (2.30) into the superspace and restore the dependence on the odd coordinates. In this way, one finds a general form of the two-particle $S L(2 \mid \mathcal{N})$ invariant evolution kernel

$$
\begin{aligned}
{\left[\mathbb{H}_{12}\right.} & \cdot \mathbb{O}]\left(Z_{1}, Z_{2}, \ldots, Z_{L}\right) \\
& =\int_{0}^{1} d \alpha \int_{0}^{\bar{\alpha}}(\bar{\alpha} \bar{\beta})^{2 j-2} \varphi\left(\frac{\alpha \beta}{\bar{\alpha} \bar{\beta}}\right) \mathbb{O}\left(\bar{\alpha} Z_{1}+\alpha Z_{2}, \beta Z_{1}+\bar{\beta} Z_{2}, \ldots, Z_{L}\right)
\end{aligned}
$$

where $j=-1 / 2$ is the conformal spin of the chiral superfield $\Phi(Z)$ entering 
into the light-cone operator (3.12). Here the kernel $\mathbb{H}_{12}$ acts on the coordinates of the superfields $\Phi\left(Z_{1}\right)$ and $\Phi\left(Z_{2}\right)$ and displaces them simultaneously along bosonic and fermionic directions in the superspace $Z=\left(z, \theta^{A}\right)$.

The evolution kernel (3.21) involves an arbitrary function $\varphi$ of the harmonic ratios. Its form is not fixed by superconformal invariance and is determined by the dynamics of the underlying SYM theory. The one-loop calculation of super-Feynman graphs, displayed in Fig. 4, gives $\varphi(\xi) \sim \delta(\xi)$. This suggests that the two-particle kernel looks like [24]

$$
\begin{aligned}
\mathbb{V}_{12} \mathbb{O}\left(Z_{1}, \ldots, Z_{L}\right) & =\int_{0}^{1} \frac{d \alpha}{\bar{\alpha} \alpha^{2}}\left\{2 \alpha^{2} \mathbb{O}\left(Z_{1}, Z_{2}, \ldots, Z_{L}\right)\right. \\
& \left.-\mathbb{O}\left(\alpha Z_{1}+\bar{\alpha} Z_{2}, Z_{2}, \ldots, Z_{L}\right)-\mathbb{O}\left(Z_{1}, \alpha Z_{2}+\bar{\alpha} Z_{1}, \ldots, Z_{L}\right)\right\} .
\end{aligned}
$$

This relation admits an obvious generalization to $\mathbb{V}_{k, k+1}$. However, Eq. (3.22) cannot be identified as the two-particle dilatation operator since the integral is divergent for $\alpha \rightarrow 0$. The divergence arises due to a negative value of conformal spin $j=-1 / 2$ of the light-cone superfield - see Eqs. (3.5) and (3.8) - which can be traced back to the fact that the lowest component of the superfield is a nonlocal operator $\partial_{+}^{-1} A(z)$. This means that expanding the nonlocal operator $\mathbb{O}\left(Z_{1}, \ldots, Z_{L}\right)$ around the origin $Z_{1}=\ldots=Z_{L}=0$, in terms of local operators one obtains both Wilson operators and "spurious" operators involving nonlocal fields $\partial_{+}^{-1} A(0), A_{+}(0)$ and $\partial_{+}^{-1} \bar{\lambda}(0)$. To eliminate the latter it is sufficient to subtract the two first terms in the expansion of the superfield $\Phi(Z)$ around $Z=0$ :

$$
\Phi(Z) \rightarrow \Phi^{\mathrm{W}}(Z)=\Phi(Z)-\Phi(0)-Z \cdot \partial_{Z} \Phi(0),
$$

where the superscript indicates that the superfield $\Phi^{\mathrm{W}}(Z)$ does not generate nonlocal fields. Let us define the operator $\Pi$ that projects the nonlocal operator (3.12) onto the subspace of Wilson operators

$$
\mathbb{O}^{\mathrm{W}}\left(Z_{1}, \ldots, Z_{L}\right)=\Pi \mathbb{O}\left(Z_{1}, \ldots, Z_{L}\right)=\operatorname{tr}\left\{\Phi^{\mathrm{W}}\left(Z_{1}\right) \cdots \Phi^{\mathrm{W}}\left(Z_{L}\right)\right\},
$$

so that $\Pi^{2}=\Pi$. Since Wilson operators mix among themselves and cannot mix with spurious operators (but the opposite is possible!), one expects that nonlocal operators $\mathbb{O}^{\mathrm{w}}\left(Z_{1}, \ldots, Z_{L}\right)$ evolve autonomously under the RG transformations. Indeed, decomposing the operators as

$$
\mathbb{O}\left(Z_{1}, \ldots, Z_{L}\right)=\mathbb{O}^{\mathrm{W}}\left(Z_{1}, \ldots, Z_{L}\right)+(1-\Pi) \mathbb{O}^{\text {spur }}\left(Z_{1}, \ldots, Z_{L}\right)
$$

and substituting this relation into (3.22), one finds that the operators $\mathbb{O}^{\mathrm{W}}\left(Z_{1}, \ldots, Z_{L}\right)$ obey the same relation (3.22) but with the two-particle kernel 
replaced by $[24]$

$$
\mathbb{V}_{12} \rightarrow \mathbb{H}_{12}^{\mathrm{W}}=\Pi \mathbb{V}_{12} \Pi
$$

It is straightforward to verify that the integral operator $\mathbb{H}_{12}^{\mathrm{W}}$ is well defined, that is, the above-mentioned divergences for $\alpha \rightarrow 0$ do not appear in the expression for $\mathbb{H}_{12}^{\mathrm{W}} \mathbb{O}\left(Z_{1}, \ldots, Z_{L}\right)$. Thus, as far as the renormalization of Wilson operators is concerned, the one-loop dilatation operator is given in the multicolor limit by Eq. (3.18) with the two-particle kernel $\mathbb{H}_{k, k+1}^{\mathrm{W}}$ defined in (3.26).

The expression obtained for the dilatation operator does not exhibit an explicit dependence on $\mathcal{N}$. This dependence enters entirely through the number of odd directions in the superspace, $Z=\left(z, \theta^{A}\right)$. This means that for nonlocal light-cone operators (3.12) the dilatation operator has the same, universal form in $\mathcal{N}=0, \mathcal{N}=1, \mathcal{N}=2$ and $\mathcal{N}=4$ SYM theories on the light-cone. The important difference between these theories is that for $\mathcal{N}=4$ the operators (3.12) generate all Wilson operators of the twist $L$, whereas for $\mathcal{N} \leq 2$ they capture only a subsector of Wilson operators. To describe the remaining operators, one has to consider single-trace operators built from both chiral and anti-chiral superfields, $\Phi$ and $\bar{\Phi}$, respectively. Remarkably enough, the dilatation operator for such mixed nonlocal operators can be deduced from the dilatation operator (3.22) in maximally supersymmetric $\mathcal{N}=4$ theory via the truncation procedure described above.

\subsection{Dilatation operator as a $S L(2 \mid \mathcal{N})$ Heisenberg spin chain}

Let us consider the properties of the one-loop dilatation operator (3.22) in more detail and demonstrate how one can use the obtained expressions to evaluate the anomalous dimensions of various Wilson operators in $\mathcal{N}$-extended SYM theories.

To begin with, consider (3.22) for $\mathcal{N}=0$. In this case, the nonlocal operator (3.12) is reduced to the product of holomorphic components of gauge fields $\partial_{+}^{-1} A(z)$. To avoid spurious operators it is convenient to go over to the nonlocal light-cone operators

$$
\mathbb{O}_{g}\left(z_{1}, \ldots, z_{L}\right)=\left(\partial_{z_{1}} \ldots \partial_{z_{L}}\right)^{2} \mathbb{O}\left(z_{1}, \ldots, z_{L}\right)=\operatorname{tr}\left\{\partial_{+} A\left(z_{1}\right) \cdots \partial_{+} A\left(z_{L}\right)\right\} .
$$

Remember that we have adopted the light-like axial gauge $A_{+}(x)=0$. In the covariant formulation the field $\partial_{+} A(z)$ is replaced by $n^{\mu}\left(F_{\mu \perp}(z)-\right.$ $\left.i \tilde{F}_{\mu \perp}(z)\right)$. Recall that the field $A(z)$ carries the helicity -1 and, therefore, $\mathbb{O}_{g}\left(z_{1}, \ldots, z_{L}\right)$ is the generating function for pure gluonic Wilson operators 
of maximal helicity $-L$.

Substituting Eq. (3.27) in (3.22) one gets

$$
\begin{aligned}
{\left[\mathbb{H}_{12}^{g}\right.} & \left.\cdot \mathbb{O}^{g}\right]\left(z_{1}, z_{2}, \ldots, z_{L}\right)=\int_{0}^{1} \frac{d \alpha}{\alpha}\left\{2 \mathbb{O}^{g}\left(\bar{\alpha} z_{1}+\alpha z_{2}, z_{2}, \ldots, z_{L}\right)\right. \\
& \left.-\bar{\alpha}^{2} \mathbb{O}^{g}\left(\bar{\alpha} z_{1}+\alpha z_{2}, z_{2}, \ldots, z_{L}\right)-\bar{\alpha}^{2} \mathbb{O}^{g}\left(z_{1}, \bar{\alpha} z_{2}+\alpha z_{1}, \ldots, z_{L}\right)\right\} .
\end{aligned}
$$

This result should be compared with the similar expression for the dilatation operator for quarks with the aligned helicities, Eq. (2.26). The only difference is in the power of $\bar{\alpha}$ in front of the second and third terms. In general, this power is determined by the conformal spin of the field, $\sim \bar{\alpha}^{2 j-1}$. For the quark field, one has $j_{q}=1$, whereas for the gauge field strength, $j_{g}=3 / 2$, and for scalar fields, $j_{s}=1 / 2$.

To diagonalize the dilatation operator, one has to repeat the same steps as described in Sect. 2.2 in the case of aligned helicity quark operators. Namely, one substitutes

$$
\mathbb{O}^{g}\left(z_{1}, z_{2}, \ldots, z_{L}\right) \rightarrow\left(z_{1}-z_{2}\right)^{n} \equiv z_{12}^{n}
$$

into (3.28) and obtains

$$
\mathbb{H}_{12}^{g} z_{12}^{n}=2[\psi(n+3)-\psi(1)] z_{12}^{n} .
$$

This relation defines the anomalous dimension of the conformal operator built from two gauge fields of the same helicity

$$
\mathcal{O}_{g}(0)=\partial_{+} A(0)\left(i \partial_{+}\right)^{n} \partial_{+} A(0)+\ldots .
$$

Here ellipses stand for terms involving total derivatives. The conformal $S L(2 ; \mathbb{R})$ invariance fixes them uniquely and allows one to write down a closed expression in terms of Gegenbauer polynomials [15]. The conformal spin of the operator (3.30) equals $J_{12}=n+2 j_{g}=n+3$ and, therefore, the two-particle dilatation operator (3.28) can be written in the $S L(2)$ invariant form

$$
\mathbb{H}_{12}^{g}=2\left[\psi\left(J_{12}\right)-\psi(1)\right]
$$

with $J_{12}$ being the two-particle conformal spin. In contrast with the quark operator, the corresponding $S L(2 ; \mathbb{R})$ representation has $j_{g}=3 / 2$.

Combining together (3.31) and (3.18), we conclude that the dilatation operator in the sector of maximal helicity gluon operators can be identified as the Hamiltonian of the $S L(2 ; \mathbb{R})$ Heisenberg magnet. The length of the spin chain is equal to $L$ and the value of the spin in each site is given by the conformal spin of the gauge strength tensor $j_{g}=3 / 2$. Notice that 
the maximal-helicity gluonic operators evolve autonomously and the corresponding one-loop mixing matrix is sensitive to the gluonic sector of the gauge theory only. This implies that the one-loop dilatation operator in the sector of maximal-helicity gluonic operators is the same in all $\mathcal{N}$-extended SYM theories.

The Wilson operators built from six scalars $\phi^{A B}(x)$ with no derivatives provide another limiting case which reflects the specifics of the $\mathcal{N}=4 \mathrm{SYM}$ theory. It proves convenient to introduce their linear combinations [67]

$$
\phi_{j}(x)=\frac{1}{2 \sqrt{2}} \Sigma_{j}^{A B} \bar{\phi}_{A B}(x)
$$

where $j=1, \ldots, 6$ and $\Sigma_{j}^{A B}$ are the chiral blocks of Dirac matrices in sixdimensional Euclidean space. Then, the composite scalar operators look like

$$
O_{j_{1} j_{2} \ldots j_{L}}=\operatorname{tr}\left\{\phi_{j_{1}}(0) \phi_{j_{2}}(0) \ldots \phi_{j_{L}}(0)\right\}
$$

To one-loop order, these operators evolve autonomously under the RG transformations and the corresponding dilatation operator has the form (3.18) with the two-particle kernel given by a finite-dimensional matrix

$$
\mathbb{H}_{12} \phi_{j_{1}}(0) \phi_{j_{2}}(0)=\sum_{j_{1}^{\prime}, j_{2}^{\prime}} V_{j_{1} j_{2}}^{j_{1}^{\prime} j_{2}^{\prime}} \phi_{j_{1}^{\prime}}(0) \phi_{j_{2}^{\prime}}(0) \text {. }
$$

The scalar field can be obtained from the $\mathcal{N}=4$ superfield (3.5) by means of a simple projector

$$
\phi_{j}(x)=\left.\frac{i}{2 \sqrt{2}} \Sigma_{j}^{A B} \partial_{\theta^{A}} \partial_{\theta^{B}} \Phi\left(x, \theta^{A}, 0\right)\right|_{\theta^{A}=0} .
$$

Using this projection, the mixing matrix $V_{j_{1} j_{2}}^{j_{1}^{\prime} j_{2}^{\prime}}$ can be extracted from the general expression for the $\mathcal{N}=4$ one-dilatation operator given in Eqs. (3.26) and (3.22):

$$
V_{j_{1} j_{2}}^{j_{1}^{\prime} j_{2}^{\prime}}=-\left.\frac{1}{8}\left(\Sigma_{j_{1}}^{A B} \partial_{\theta_{1}^{A}} \partial_{\theta_{1}^{B}}\right)\left(\Sigma_{j_{2}}^{C D} \partial_{\theta_{2}^{C}} \partial_{\theta_{2}^{D}}\right)\left[\mathbb{H}_{12} \cdot \mathbb{O}\right]\left(Z_{1}, Z_{2}\right)\right|_{Z_{1}=Z_{2}=0} .
$$

The calculation of fermionic derivatives then yields

$$
V_{j_{1} j_{2}}^{j_{1}^{\prime} j_{2}^{\prime}}=\delta_{j_{1}}^{j_{1}^{\prime}} \delta_{j_{2}}^{j_{2}^{\prime}}+\frac{1}{2} \delta_{j_{1} j_{2}} \delta^{j_{1}^{\prime} j_{2}^{\prime}}-\delta_{j_{1}}^{j_{2}^{\prime}} \delta_{j_{2}}^{j_{1}^{\prime}}
$$

This matrix acts on the isotopic indices of two neighboring scalar fields in (3.33) and has the size $6^{2} \times 6^{2}$. Hence, the one-loop dilatation operator for the scalar composite operators (3.33) is given by the $6^{L} \times 6^{L}$-matrix. As in the 
previous case it has a hidden symmetry - this matrix can be mapped into the Hamiltonian of the completely integrable Heisenberg $S O(6) \sim S U(4)$ spin chain of length $L[25]$.

We see that in the $\mathcal{N}=4 \mathrm{SYM}$ theory, the integrability phenomenon occurs in two different sectors of Wilson operators. In both cases, one encounters the Heisenberg spin chain of length $L$ but with different symmetry groups. For the maximal helicity Wilson operators with arbitrary number of derivatives, the $S L(2 ; \mathbb{R})$ symmetry group arises and it is related to conformal symmetry on the light-cone. For scalar operators with no derivatives, the arising $S U(4)$ symmetry group is related to the $R$-symmetry; that is, rotations in the isotopic space. From the point of view of the superspace, $Z=\left(z, \theta^{A}\right)$, the two groups act separately on the bosonic and fermionic coordinates of the superfields and as such they are subgroups of a bigger, $S L(2 \mid \mathcal{N})$ group. Since the $S L(2 ; \mathbb{R})$ and $S O(6)$ spin chains emerge as special limits of a general expression for the one-loop dilatation operator Eq. (3.22), a question arises about integrability properties of the latter operator as a whole. Quite naturally, the one-loop dilatation operator can be mapped into a Hamiltonian of the Heisenberg spin magnet with the $S L(2 \mid \mathcal{N})$ symmetry group. The latter is defined in (3.18) with the two-particle kernels given by the familiar expression $[24,71]$

$$
\mathbb{H}_{12}^{\mathrm{W}}=2\left[\psi\left(J_{12}\right)-\psi(1)\right], \quad J_{12}\left(J_{12}-1\right)=L_{12}^{2}
$$

in which $J_{12}$ is the two-particle $S L(2 \mid \mathcal{N})$ spin and the quadratic Casimir was introduced in (3.20).

For $\mathcal{N}=4$ this (super) spin chain describes renormalization of arbitrary Wilson operators in the underlying SYM theory built from "good" components of the fundamental fields. For $\mathcal{N} \leq 2$, it covers only a special subsector of Wilson operators built from fundamental fields entering into the expression for the chiral superfield, Eq. (3.8). In particular, at $\mathcal{N}=0$ this sector corresponds to maximal helicity gluonic operators. For the remaining Wilson operators generated by mixed products of chiral and antichiral superfields, the dilatation operator does not exhibit integrability properties. As was illustrated in Sect. 2.5 in the case of non-maximal helicity quark operators, the additional terms in the dilatation operator responsible for breaking the integrability generate a mass gap in the spectrum of the anomalous dimensions.

The findings of Ref. [23] suggest that in the $\mathcal{N}=4$ theory, not only quasi-partonic operators, addressed above, but all operators obey integrable renormalization group equations. Thus the spin chain discussed above gets 
enhanced to the $S U(2,2 \mid 4)$ superchain. As we have emphasized in Sect. 2.7 this is a very nontrivial statement in the QCD context. It suggests that operators of different twists are intertwined in a very subtle manner. The superconformal symmetry does indeed relate operators of different twists. However, this is not enough to conclude that the anomalous dimensions of non-quasipartonic operators are expressed via the quasipartonic operators, since this is not the case in the most straightforward supersymmmetric generalization of QCD, the $\mathcal{N}=1 \mathrm{SYM}$. The presence of the elementary scalar fields in the $\mathcal{N}=4$ model does matter, so that multiple full ${ }^{\mathrm{j}}$ supersymmetric transformations of the operator $\phi^{i} D_{+}^{N} \phi^{i}$, having the integrable form of the anomalous dimension (3.29) will yield combinations of operators with different twists but having the same anomalous dimension. This is not the case in QCD where anomalous dimensions of different twist operators are believed to be dynamically independent. This issue deserves thorough further study.

There have been several recent related developments that we cannot address here in detail. In particular, the anomalous dimension matrices in different autonomous sectors in $\mathcal{N}=4 \mathrm{SYM}$ were studied in Refs. [73-76], mostly in the different subsectors of scalar operators. Another very important question is whether the integrability of the dilatation operator persists at higher orders in the loop expansion. Several positive demonstrations that this does happen have been given in certain scalar subsectors [77-79]. To extend these findings to the full dilatation operator, the analysis of the $S L(2)$ subsector has to be performed. The two-particle part of the corresponding dilatation operator was calculated in Refs. [80] up to three-loop order.

Ultimately, one has to understand what symmetry of the quantum field theory is responsible for the appearance of integrability [81,82] and what part of it survives after decreasing the amount of supersymmetry [83-85], as we explained for one particular case above.

\section{High Energy Scattering}

In the previous section, we have considered the perturbative QCD dynamics of partons constrained to small transverse separations, which can be achieved by considering "hard" scattering processes in generalized Bjorken kinematics. When the transverse distance becomes smaller than any other scale in the process, the corresponding dependence can be treated by renormalization-group methods and disappears from the dynamics. The problem becomes essentially one dimensional: the relevant degrees of free-

${ }^{\mathrm{j}}$ I.e., not restricted to the light-cone as in Eq. 3.7. 
dom correspond to partons "living" on the light-ray and the interaction between them amounts to displacement along the light-ray, with a certain weight-function. We were able to analyze this interaction using a Hamiltonian formulation and found its hidden integrability property. The scale dependence in this case corresponds to "time" evolution in the quantummechanical picture.

The present section is devoted to perturbative QCD dynamics in the high-energy limit. In this case, the invariant energy $s=\left(p_{A}+p_{B}\right)^{2}$ of colliding hadrons $A$ and $B$ is considered to be the largest scale and, in the limit $s \rightarrow \infty$, the energy dependence corresponds to a renormalization group flow for a dynamical system "living" in the two transverse dimensions orthogonal to the scattering plane. The relevant degrees of freedom correspond to reggeized gluons (see below) with assigned transverse coordinates, and the interaction between them leads to a displacement in the transverse plane. Compared to the "hard" scattering situation, the longitudinal and transverse degrees of freedom change places, and the dynamics becomes two dimensional in the transverse plane instead of being one dimensional in a given light-ray direction as in the former case. In what follows, we will sketch the Hamiltonian approach to high-energy scattering and establish its hidden symmetry properties. The main result of this analysis is that the system of interacting reggeized gluons can be mapped into a quantum XXX Heisenberg magnet with the $S L(2, \mathbb{C})$ symmetry group [7-9]. This means thatthe high-energy asymptotics of scattering amplitudes in QCD in a certain limit can be calculated using the Quantum Inverse Scattering Method [32].

\subsection{The BFKL Hamiltonian}

The high-energy asymptotics of the scattering amplitude $\mathcal{A}(s, t)$ is studied most conveniently through the Mellin representation which corresponds to an expansion in partial waves with complex angular momentum

$$
\mathcal{A}(s, t)=i s \int_{\delta-i \infty}^{\delta+i \infty} \frac{d \omega}{2 \pi i} s^{\omega} \widetilde{\mathcal{A}}(\omega, t) .
$$

Here, the integration contour goes to the right of all singularities of $\widetilde{\mathcal{A}}(\omega, t)$ on the complex $\omega$-plane. The high-energy asymptotics of $\mathcal{A}(s, t)$ is determined by the singularities of the partial wave amplitudes: If $\widetilde{\mathcal{A}}(\omega, t) \sim 1 /\left(\omega-\omega_{0}(t)\right)$ then $\mathcal{A}(s, t) \sim i s^{1+\omega_{0}(t)}$. Poles in the $\omega$-plane are called reggeons, and the position of the pole as a function of the transfered momentum, $\omega_{0}(t)$, is called the reggeon trajectory. From the point of view of QCD perturbation theory, finding the high-energy asymptotics of the amplitude corresponds 
to the resummation of logarithms of the energy $\sim\left(\alpha_{s} \ln s\right)^{m}$. By virtue of the Mellin transformation (4.1), the expansion over $\left(\alpha_{s} \ln s\right)^{m}$ is traded for the expansion over $\left(\alpha_{s} / \omega\right)^{m}$ with coefficients that depend on $t$, and the power-like energy-dependence arises because the resummed expression for $\widetilde{\mathcal{A}}\left(\alpha_{s} / \omega, t\right)$ develops a nontrivial singularity for positive $\omega=\omega_{0}(t)$ in the sum to all orders in $\alpha_{s}$.

The partial wave amplitude $\widetilde{\mathcal{A}}(\omega, t)$ can be written in the so-called impactparameter representation

$$
\begin{aligned}
\widetilde{\mathcal{A}}(\omega, t) & =\int d^{2} b_{0} \mathrm{e}^{i\left(q b_{0}\right)} \int d^{2} b d^{2} b^{\prime} \Phi_{A}\left(\vec{b}-\vec{b}_{0}\right) T_{\omega}\left(\vec{b}, \overrightarrow{b^{\prime}}\right) \Phi_{B}\left(\overrightarrow{b^{\prime}}\right) \\
& \equiv \int d^{2} b_{0} \mathrm{e}^{i\left(q b_{0}\right)}\left\langle\Phi\left(b_{0}\right)\left|\mathbb{T}_{\omega}\right| \Phi(0)\right\rangle
\end{aligned}
$$

where the impact factors $\Phi_{A}(\vec{b})$ and $\Phi_{B}\left(\overrightarrow{b^{\prime}}\right)$ stand for the parton (gluon) distributions as functions of transverse coordinates $\vec{b}=\left\{\vec{b}_{1}, \vec{b}_{2}, \ldots, \vec{b}_{n}\right\}$ and $\vec{b}^{\prime}=\left\{\vec{b}_{1}^{\prime}, \vec{b}_{2}^{\prime}, \ldots \vec{b}_{m}^{\prime}\right\}$ in the colliding hadrons $A$ and $B$, respectively, and $T_{\omega}\left(\vec{b}, \overrightarrow{b^{\prime}}\right)$ is the scattering (partial wave) amplitude for a given parton configuration. The existence of such a representation follows from the space-time picture of the high-energy collisions: The two Lorentz-contracted hadron discs approach each other at the speed of light and the interaction between them takes a finite time in the center-of-mass frame. The preparation of the initial states is separated in time from the interaction between hadrons and, therefore, the transverse coordinates of parton constituents in hadrons can be considered as "frozen" during the interaction. Note that the factorized form in (4.2) implies that the structure of singularities in the complex $\omega$ plane does not depend on the parton distribution in hadrons, but rather on general properties of the gluon interaction in the $t$-channel.

A nontrivial analysis [6] shows that the interaction effects modify the propagators of the $t$-channel gluons in such a way that they develop their own Regge trajectory; such $t$-channel gluons "dressed" by virtual corrections are called reggeized gluons. The reggeized gluons with given transverse coordinates provide one with the relevant degrees of freedom for the study of high energy interactions and play a rôle similar to that of the fundamental fields with given light-ray coordinates for light-cone dominated processes. In the course of interaction with each other, the reggeized gluons change their two-dimensional transverse coordinates and the color charge. The partial waves $T_{\omega}\left(\vec{b}, \overrightarrow{b^{\prime}}\right)$ can be classified according to the number of reggeized gluons propagating in the $t$-channel; the minimum number of two gluons is required in order to get a colorless exchange. Moreover, it can be shown that retain- 
ing only two $t$-channel reggeized gluons corresponds to the resummation of energy logarithms to the leading logarithmic accuracy. In this approximation the amplitude $T_{\omega}\left(\vec{b}_{1}, \vec{b}_{2} ; \vec{b}_{1}^{\prime}, \vec{b}_{2}^{\prime}\right)$ depends on four transverse coordinates and satisfies a Bethe-Salpeter-like equation - the so-called BFKL equation - in the operator form

$$
\omega \mathbb{T}_{\omega}=\mathbb{T}_{\omega}^{(0)}+\frac{\alpha_{s} N_{c}}{\pi} \mathbb{H}_{\mathrm{BFKL}} \mathbb{T}_{\omega}
$$

where $\mathbb{T}_{\omega}^{(0)}$ corresponds to the free exchange of two gluons. The solution of this equation can formally be written as

$$
\mathbb{T}_{\omega}=\left(\omega-\frac{\alpha_{s} N_{c}}{\pi} \mathbb{H}_{\mathrm{BFKL}}\right)^{-1} \mathbb{T}_{\omega}^{(0)},
$$

whence one concludes that the singularities of $\mathbb{T}_{\omega}$ in the $\omega$-plane are determined by the eigenvalues of the BFKL operator

$$
\left[\mathbb{H}_{\mathrm{BFKL}} \cdot \Psi_{\alpha}\right]\left(\vec{b}_{1}, \vec{b}_{2}\right)=E_{\alpha} \Psi_{\alpha}\left(\vec{b}_{1}, \vec{b}_{2}\right),
$$

with $\alpha$ enumerating the solutions. The high-energy behavior of the scattering amplitude is governed by the right-most singularity of $\mathbb{T}_{\omega}$, which corresponds to the maximal eigenvalue $\max _{\alpha} E_{\alpha}$. Equation (4.5) has the form of a Schrödinger equation for a system of two interacting particles on the two-dimensional plane. Such particles can be identified as reggeized gluons and the eigenstates $\Psi_{\alpha}\left(\vec{b}_{1}, \vec{b}_{2}\right)$ have the meaning of the wave functions of the color-singlet compound states built from two reggeized gluons.

The BFKL operator $\mathbb{H}_{\text {BFKL }}$ has a number of remarkable properties which allow one to solve the Schrödinger equation (4.5) exactly [86, 87]. First of all, $\mathbb{H}_{\mathrm{BFKL}}$ splits into the sum of two operators acting on the holomorphic and the antiholomorphic coordinates

$$
\vec{b}_{j}=\left\{x_{j}, y_{j}\right\} \quad \Longrightarrow \quad z_{j}=x_{j}+i y_{j}, \quad \bar{z}_{j}=x_{j}-i y_{j},
$$

(with $j=1,2$ ) on the transverse plane:

$$
\mathbb{H}_{\mathrm{BFKL}}=\mathcal{H}_{2}+\overline{\mathcal{H}}_{2}
$$

with

$$
\mathcal{H}_{2}=\partial_{z_{1}}^{-1} \ln \left(z_{12}\right) \partial_{z_{1}}+\partial_{z_{2}}^{-1} \ln \left(z_{12}\right) \partial_{z_{2}}+\ln \left(\partial_{z_{1}} \partial_{z_{2}}\right)-2 \psi(1),
$$

where $z_{12}=z_{1}-z_{2}$ and $\overline{\mathcal{H}}_{2}$ is given by a similar expression in the $\bar{z}$-sector.

Another remarkable property of $\mathbb{H}_{\mathrm{BFKL}}$ is that it is invariant under the conformal $S L(2 ; \mathbb{C})$ transformations of the reggeon coordinates on the plane 


$$
z_{k} \rightarrow \frac{a z_{k}+b_{k}}{c z_{k}+d}, \quad(a d-b c=1) .
$$

Consider the generators of the holomorphic $S L(2, \mathbb{C})$ transformations

$$
L_{k,-}=-\partial_{z_{k}}, \quad L_{k, 0}=z_{k} \partial_{z_{k}}, \quad L_{k,+}=z_{k}^{2} \partial_{z_{k}}
$$

and the corresponding antiholomorphic generators $\bar{L}_{k,-}, \bar{L}_{k, 0}$ and $\bar{L}_{k,+}$ given by similar expressions with $z_{k}$ replaced by $\bar{z}_{k}$, with $k=1,2$ enumerating particles. By inspection one finds that $\mathbb{H}_{\mathrm{BFKL}}$ commutes with all two-particle generators

$$
\left[\mathbb{H}_{\mathrm{BFKL}}, L_{1, a}+L_{2, a}\right]=\left[\mathbb{H}_{\mathrm{BFKL}}, \bar{L}_{1, a}+\bar{L}_{2, a}\right]=0
$$

with $a=+,-, 0$. This implies that $\mathbb{H}_{\mathrm{BFKL}}$ only depends on the two-particle Casimir operators of the $S L(2, \mathbb{C})$ group

$$
L_{12}^{2}=-\left(z_{1}-z_{2}\right)^{2} \partial_{z_{1}} \partial_{z_{2}}, \quad \bar{L}_{12}^{2}=-\left(\bar{z}_{1}-\bar{z}_{2}\right)^{2} \partial_{\bar{z}_{1}} \partial_{\bar{z}_{2}},
$$

and leads to $\mathcal{H}_{2}=\mathcal{H}_{2}\left(L_{12}^{2}\right)$ and $\overline{\mathcal{H}}_{2}=\overline{\mathcal{H}}_{2}\left(\bar{L}_{12}^{2}\right)$. As a consequence, solutions of the Schrödinger equation (4.5) have to be eigenstates of the Casimir operators

$$
L_{12}^{2} \Psi_{n, \nu}=h(h-1) \Psi_{n, \nu}, \quad \bar{L}_{12}^{2} \Psi_{n, \nu}=\bar{h}(\bar{h}-1) \Psi_{n, \nu} .
$$

Here a pair of complex conformal spins is introduced

$$
h=\frac{1+n}{2}+i \nu \quad \text { and } \quad \bar{h}=\frac{1-n}{2}+i \nu
$$

with a non-negative integer $n$ and real $\nu$ that specify the irreducible (principal series) representation of the $S L(2, \mathbb{C})$ group to which $\Psi_{n, \nu}$ belongs. The solutions to Eqs. (4.13) read as

$$
\Psi_{n, \nu}\left(b_{1}, b_{2}\right)=\left(\frac{z_{12}}{z_{10} z_{20}}\right)^{(1+n) / 2+i \nu}\left(\frac{\bar{z}_{12}}{\bar{z}_{10} \bar{z}_{20}}\right)^{(1-n) / 2+i \nu},
$$

where $z_{j k}=z_{j}-z_{k}$ and $b_{0}=\left(z_{0}, \bar{z}_{0}\right)$ is the collective coordinate, reflecting the invariance of $\mathbb{H}_{\mathrm{BFKL}}$ under translations. We recall that the solution (4.15) defines the wave function of the color-singlet compound state, which is built from two reggeized gluons with the coordinates $b_{1}=\left(z_{1}, \bar{z}_{1}\right)$ and $b_{2}=\left(z_{2}, \bar{z}_{2}\right)$. The integer $n$ fixes the two-dimensional Lorentz spin of the state, the real valued $\nu$ gives the scaling dimension $\ell=1+2 i \nu$, and the two-dimensional vector $b_{0}$ sets up the center-of-mass coordinate of the state. 
To find the eigenvalues in Eq. [4.5), one substitutes the wave function (4.15) into the Schrödinger equation (4.5) and uses the explicit form of the BFKL kernel leading to [6]

$$
E_{n, \nu}=2 \psi(1)-\psi\left(\frac{n+1}{2}+i \nu\right)-\psi\left(\frac{n+1}{2}-i \nu\right) .
$$

Its maximal value, $\max E_{n, \nu}=4 \ln 2$, corresponds to $n=\nu=0$, or equivalently $h=\bar{h}=1 / 2$. It defines the position of the right-most singularity of the partial wave amplitude and is translated into the asymptotic behavior of the scattering amplitude in the leading logarithmic approximation,

$$
\mathcal{A}(s, t) \sim i s^{1+\frac{\alpha_{s} N_{c}}{\pi} 4 \ln 2},
$$

known as the BFKL pomeron. ${ }^{\mathrm{k}}$

Using (4.16) one can reconstruct the operator form of the BFKL kernel $\mathbb{H}_{\mathrm{BFKL}}$ on the representation space of the principal series of the $S L(2, \mathbb{C})$ group

$$
\mathbb{H}_{\mathrm{BFKL}}=\frac{1}{2}\left[H\left(J_{12}\right)+H\left(\bar{J}_{12}\right)\right], \quad H(j)=2 \psi(1)-\psi(j)-\psi(1-j),
$$

where, as before, the two-particle spins are defined as $L_{12}^{2}=J_{12}\left(J_{12}-1\right)$ and $\bar{L}_{12}^{2}=\bar{J}_{12}\left(\bar{J}_{12}-1\right)$. Notice that we already encountered the similar Hamiltonian in Sect. 2 and found that it gives rise to complete integrability for the three-particle evolution equations for the helicity $-3 / 2$ baryon distribution amplitudes. It turns out that the BFKL kernel (4.18) has the same hidden integrability. In order to appreciate this, we have to consider states containing more than two particles.

\subsection{Multireggeon compound states}

In the leading logarithmic approximation, the scattering amplitude (4.17) grows as a power of the energy and violates unitarity constraints. To restore unitarity, subleading corrections must be included. Going beyond the leading logarithmic approximation, one has to take into account contributions to the scattering amplitude in Eq. (4.2) with more than two reggeized gluons propagating in the $t$-channel. One can argue $[88,89]$ that, in order to restore the unitarity of the scattering amplitude in the direct $(s-$ and $t-$ ) channels, it suffices to retain contributions where the number $N$ of

\footnotetext{
${ }^{\mathrm{k}}$ Due to accumulation of the energy levels $E_{n, \nu}$ around $n=\nu=0$, the corresponding Regge singularity is not a pole but a square-root cut so that 4.17 is modified by additional $\left(\alpha_{s} \ln s\right)^{-1 / 2}$-factor.
} 
the $t$-channel gluons is conserved, i.e., neglect the three-reggeon vertices in the Regge theory language. With this assumption, the amplitude $\mathcal{A}_{N}(s, t)$ corresponding to the exchange by $N$ reggeized gluons satisfies the BartelsKwiecinski-Praszalowicz (BKP) equation $[88,90]$, which is the generalization of the Bethe-Salpeter equation (4.3) to the $N$-gluon scattering amplitude. Singularities of $\mathbb{T}_{\omega}$ in the complex $\omega$-plane are determined by the eigenvalues of the BKP operator $\mathbb{H}_{N}$. They can be thought of as energies of the $N$-gluon compound states

$$
\mathbb{H}_{N} \Psi_{N,\{q\}}\left(\vec{b}_{1}, \ldots, \vec{b}_{N}\right)=E_{N,\{q\}} \Psi_{N,\{q\}}\left(\vec{b}_{1}, \ldots, \vec{b}_{N}\right),
$$

with $\{q\}$ being some set of quantum numbers parameterizing the solutions, and $\vec{b}_{n}$ the two-dimensional transverse positions of $n$-th reggeized gluon.

In the large $N_{c}$ limit, the relevant Feynman diagrams have the topology of a cylinder and $\mathbb{H}_{N}$ reduces to the sum of terms corresponding to pairwise nearest-neighbor BFKL interactions:

$$
\mathbb{H}_{N}=\frac{1}{2} \sum_{k=1}^{N} \mathbb{H}_{k, k+1}^{\mathrm{BFKL}}
$$

with periodic boundary conditions $\mathbb{H}_{N, N+1}^{\mathrm{BFKL}}=\mathbb{H}_{N, 1}^{\mathrm{BFKL}}$. The holomorphic separability of $\mathbb{H}_{\mathrm{BFKL}}$ implies the same property for $\mathbb{H}_{N}$

$$
\mathbb{H}_{N}=\mathcal{H}_{N}+\overline{\mathcal{H}}_{N}
$$

with the operators $\mathcal{H}_{N}$ and $\overline{\mathcal{H}}_{N}$ acting on $z$ - and $\bar{z}$-coordinates, respectively. The conformal symmetry is also inherited by them, so that each of the two-particle Hamiltonians can be written in terms of the corresponding two-particle Casimir operators

$$
\mathbb{H}_{N}=\frac{1}{2} \sum_{k=1}^{N}\left[H\left(J_{k, k+1}\right)+H\left(\bar{J}_{k, k+1}\right)\right],
$$

where $H(j)$ is given in terms of $\psi$-functions (see Eq. (4.18)).

As a consequence, the $(2+1)$-dimensional Schrödinger equation (4.19) can be replaced by the system of holomorphic and antiholomorphic $(1+1)-$ dimensional Schrödinger equations for the Hamiltonians $\mathcal{H}_{N}$ and $\overline{\mathcal{H}}_{N}$. This allows one to expand the wave function of the $N$-reggeon state over the eigenstates of $H_{N}$ and $\bar{H}_{N}$ as [91]

$$
\Psi\left(\vec{b}_{1}, \ldots, \vec{b}_{N}\right)=\sum_{a, b} C_{a b} \Psi^{(a)}\left(z_{1}, \ldots, z_{N}\right) \bar{\Psi}^{(b)}\left(\bar{z}_{1}, \ldots, \bar{z}_{N}\right),
$$

where $C_{a b}$ are the mixing coefficients. 
Most remarkably, the Hamiltonian (4.20) is in fact the Hamiltonian of the $S L(2, \mathbb{C})$ Heisenberg magnet. The number of sites in the spin chain equals the number of reggeons and the corresponding spin operators are identified as six generators, $L_{k}^{ \pm}, L_{k}^{0}$ and $\bar{L}_{k}^{ \pm}, \bar{L}_{k}^{0}$, of the $S L(2, \mathbb{C})$ group. The non-compact $S L(2, \mathbb{C})$ Heisenberg magnet describes a completely integrable model. It possesses a large-enough set of mutually commuting conserved charges $q_{n}$ and $\bar{q}_{n}(n=2, \ldots, N)$ such that $\bar{q}_{n}=q_{n}^{\dagger}$ and $\left[\mathbb{H}_{N}, q_{n}\right]=\left[\mathbb{H}_{N}, \bar{q}_{n}\right]=0$. The charges $q_{n}$ are polynomials of degree $n$ in the holomorphic spin operators. They have the following form $[7,8]$

$$
q_{n}=\sum_{1 \leq j_{1}<j_{2}<\ldots<j_{n} \leq N} z_{j_{1} j_{2}} z_{j_{2} j_{3} \ldots} \ldots z_{j_{n} j_{1}} p_{j_{1}} p_{j_{2}} \ldots p_{j_{n}}
$$

with $z_{j k}=z_{j}-z_{k}$ and $p_{j}=i \partial_{z_{j}}$. The "lowest" charge $q_{2}$ is related to the total spin of the system $h$. For the principal series of the $S L(2, \mathbb{C})$ it takes the following values

$$
q_{2}=-h(h-1), \quad h=\frac{1+n_{h}}{2}+i \nu_{h},
$$

with $n_{h}$ integer and $\nu_{h}$ real. The eigenvalues of the integrals of motion, $q_{2}, \ldots, q_{N}$, form the complete set of quantum numbers parameterizing the $N$-reggeon states (4.19).

Identification of (4.20) as the Hamiltonian of the $S L(2, \mathbb{C})$ Heisenberg magnet allows one to map the $N$-reggeon states into the eigenstates of this lattice model. In spite of the fact that the noncompact $S L(2, \mathbb{C})$ Heisenberg magnet represents a generalization of the compact $S U(2)$ spin chain, very little was known about its energy spectrum until recently. The principal difficulty is that, in distinction with compact magnets, the quantum space of the $S L(2, \mathbb{C})$ magnet does not possess a highest weight - the so-called "pseudo-vacuum state" - and, as a consequence, conventional methods like the Algebraic Bethe Ansatz method [32] are not applicable. The eigenproblem (4.20) has been solved exactly in Refs. [27,28,92] using the method of the Baxter $\mathbb{Q}$-operator [93] which does not rely on the existence of a= highest weight. In this approach, it becomes possible to establish the quantization conditions for the integrals of motion $q_{3}, \ldots, q_{N}$ and to obtain an explicit form for the dependence of the energy $E_{N}$ on the integrals of motion.

Thanks to complete integrability, the "conformal blocks" $\Psi^{(a)}$ and $\bar{\Psi}^{(b)}$, Eq. (4.23), diagonalize the integrals of motion in the holomorphic and antiholomorphic sectors, respectively. The function $\Psi\left(\vec{b}_{1}, \ldots, \vec{b}_{N}\right)$ is a singlevalued function on the two-dimensional plane. It belongs to the principal series of the $S L(2, \mathbb{C})$ group, labelled by the spins $(h, \bar{h})$ defined in (4.14) 
(with $\bar{h}=1-h^{*}$ ), and is normalizable with respect to the $S L(2, \mathbb{C})$ scalar product. In contrast with $\Psi\left(\vec{b}_{1}, \ldots, \vec{b}_{N}\right)$, the chiral solutions $\Psi^{(a)}$ and $\bar{\Psi}^{(b)}$ acquire nontrivial monodromy when $\vec{b}_{k}$ encircles other particles on the plane. The quantization conditions for the integrals of motion $\boldsymbol{q}$ ensure that the monodromy cancels in the r.h.s. of (4.23). The same condition fixes (up to an overall normalization) the mixing coefficients $C_{a b}[28,91]$.

In this manner, the spectrum of the $N$-reggeon state has been calculated for $N \geq 3$ particles : For $N=3$ few low-lying states have been found in $[91,94]$ and the complete spectrum of states for $3 \leq N \leq 8$ was determined in $[27,28]$ (see also [95]). Close examination revealed the following properties. The quantized values of the charges $q_{k}$ (with $k=3, \ldots, N$ ) depend on the "hidden" set of integers $\ell=\left(\ell_{1}, \ell_{2}, \ldots, \ell_{2(N-2)}\right)$

$$
q_{k}=q_{k}\left(\nu_{h} ; n_{h}, \ell\right),
$$

where the integer $n_{h}$ and the real number $\nu_{h}$ define the total $S L(2, \mathbb{C})$ spin of the state, Eq. (4.14). As a function of $\nu_{h}$, the charges form a family of trajectories in the moduli space $\boldsymbol{q}=\left(q_{2}, q_{3}, \ldots, q_{N}\right)$ labelled by integers $n_{h}$ and $\boldsymbol{\ell}$. Each trajectory in the $q$-space induces a corresponding trajectory for the energy $E_{N}$

$$
E_{N}=E_{N}\left(\nu_{h} ; n_{h}, \ell\right) .
$$

A few examples of such trajectories for $N=3$ are shown in Fig. 5 .

Due to the complicated form of the quantization conditions, it remains unclear what the origin of trajectories is and what the physical interpretation of the integers $\ell$ is. Both questions can be answered by solving the Schrödinger equation (4.19) within the semiclassical approach [96]. One might expect a priori that this approach could be applicable only for highlyexcited states. Nevertheless, as was demonstrated in [96], the semi-classical formulae work with good accuracy throughout the whole spectrum. From the viewpoint of classical dynamics, the $N$-reggeon state describes a chain of $N$ interacting particles on the two-dimensional $\vec{b}$-plane $[17,44,97]$. The classical model inherits the complete integrability of the quantum noncompact spin magnet. Its Hamiltonian and the integrals of motion are obtained from (4.20), (4.18) and (4.24) by replacing the momentum operators by the corresponding classical functions. Since the Hamiltonian 4.20) is given by the sum of holomorphic and antiholomorphic functions, from point of view of classical dynamics the model describes two copies of one-dimensional systems "living" on the complex $z$ - and $\bar{z}$-lines. The solutions to the classical equations of motion have a rich structure and turn out to be intrinsically related 

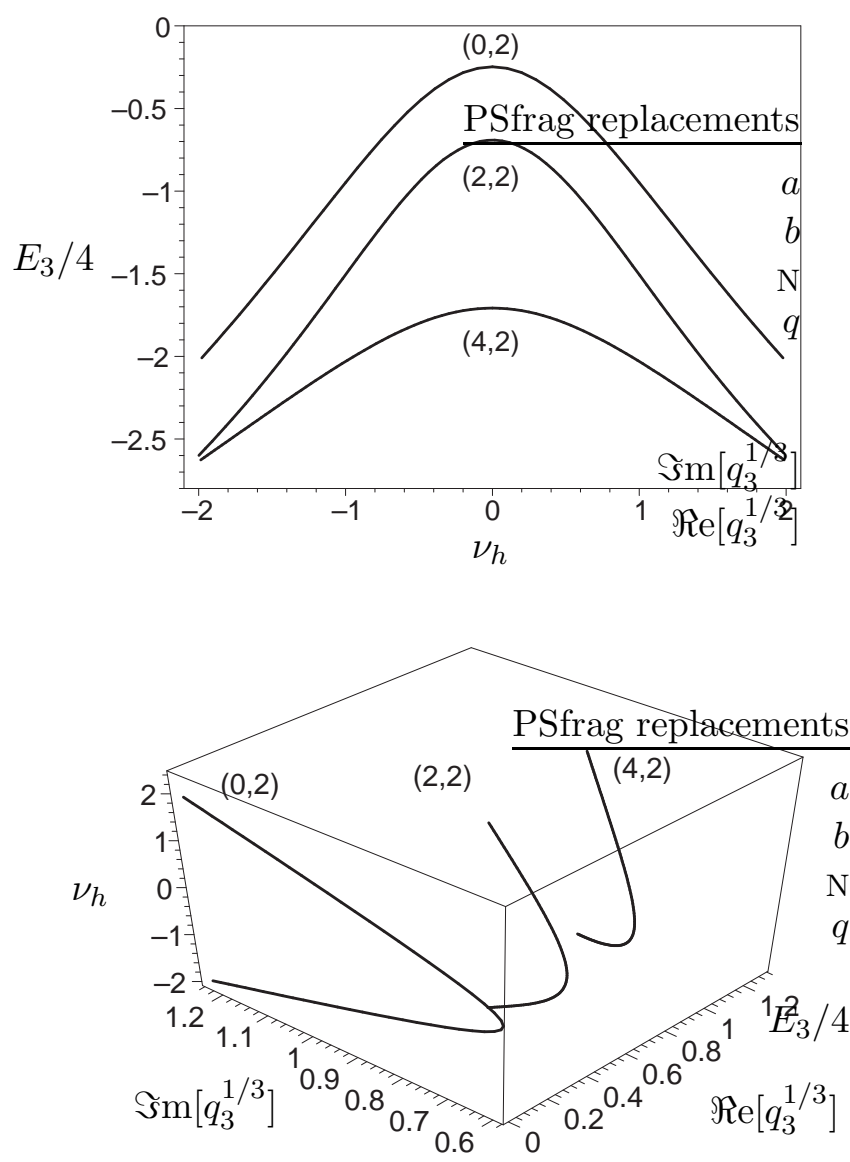

Figure 5. The dependence of the energy $E_{3}$ and the conserved charges $q_{3}$ on the total spin $h=1 / 2+i \nu_{h}$ for $n_{h}=0$. Three curves correspond to the trajectories with $\left(\ell_{1}, \ell_{2}\right)=(0,2),(2,2)$ and $(4,2)$.

to the finite-gap solutions to the nonlinear equations $[98,99]$; namely, the classical trajectories have the form of plane waves propagating in the chain of $N$ particles. Their explicit form in terms of the Riemann $\theta$-functions was established in [97] by the methods of finite-gap theory $[98,99]$. The charges $\boldsymbol{q}$ define the moduli of the finite-gap solutions and take arbitrary complex values in the classical model. Going over to the quantum model, one finds that charges $\boldsymbol{q}$ are quantized. In the semiclassical approach, their values satisfy the Bohr-Sommerfeld quantization conditions imposed on the orbits of classical motion of $N$ particles.

In a standard manner, the WKB ansatz for the eigenfunction of the model (4.20) involves the "action" function, $\Psi_{\mathrm{WKB}}\left(\vec{z}_{1}, \ldots, \vec{z}_{N}\right) \sim \exp \left(i S_{0} / \hbar\right)$. Due 
to complete integrability of the classical system, it can be defined as a simultaneous solution of the system of the Hamilton-Jacobi equations

$$
\sum_{k=1}^{N} \frac{\partial S_{0}}{\partial z_{k}}=P, \quad \mathrm{q}_{n}\left(z, \frac{\partial S_{0}}{\partial \boldsymbol{z}}\right)=q_{n}, \quad(n=2, \ldots, N),
$$

where $\boldsymbol{z}=\left(z_{1}, \ldots, z_{N}\right)$ denotes the set of holomorphic coordinates, $\mathrm{q}_{n}(\boldsymbol{z}, \boldsymbol{p})$ stands for the symbol of the operator (4.24) and $P$ is a holomorphic component of the total momentum of $N$ particles. The $\bar{z}$-dependence of $S_{0}$ is constrained by similar relations in the antiholomorphic sector. To find a general solution to Eq. (4.28), one performs a canonical transformation to the classical separated coordinates $[54,98]$

$$
\left(\vec{b}_{1}, \vec{b}_{2}, \ldots, \vec{b}_{N}\right) \stackrel{\text { SoV }}{\mapsto}\left(\vec{b}_{0}, \vec{x}_{1}, \vec{x}_{2}, \ldots, \vec{x}_{N-1}\right),
$$

with $\vec{b}_{0}$ being the center-of-mass coordinate of the system, and $\vec{x}_{n}=$ $\left(x_{n}, \bar{x}_{n}=x_{n}^{*}\right)$ the new collective (separated) coordinates. Similarly to the example in Sect. 2.4, the classical dynamics in the separated variables is determined by the spectral curve ("equal energy" condition)

$$
\Gamma_{N}: \quad y^{2}=t_{N}^{2}(x)-4 x^{2 N},
$$

where $y(x)=2 x^{N} \sinh p_{x}$ and $p_{x}$ are the momenta in the separated coordinates. Here $t_{N}(x)$ is a polynomial of degree $N$ with the coefficients defined by the holomorphic integrals of motion $q_{n}$

$$
t_{N}(x)=2 x^{N}+q_{2} x^{N-2}+\ldots+q_{N-1} x+q_{N} .
$$

The spectral curve (4.30) establishes the relation between holomorphic components of the separated coordinates, $x$ and $p_{x}$, for a given set of the charges $q_{2}, \ldots, q_{N}$.

In the separated coordinates, the solution to the Hamilton-Jacobi equations (4.28) takes the form $S_{0}\left(\vec{z}_{0}, \vec{x}_{1}, \vec{x}_{2}, \ldots, \vec{x}_{N-1}\right)=\left(\vec{P} \cdot \vec{z}_{0}\right)+\sum_{k=1}^{N-1} S_{0}\left(\vec{x}_{k}\right)$ with [98]

$$
S_{0}(\vec{x})=\int_{x_{0}}^{x} d x p_{x}+\int_{\bar{x}_{0}}^{\bar{x}} d \bar{x} \bar{p}_{\bar{x}}=2 \Re \mathrm{e} \int_{x_{0}}^{x} d x p_{x} .
$$

Here $\bar{p}_{\bar{x}}=p_{x}^{*}$ is the complex momentum defined in (4.30) and $\vec{x}_{0}$ is arbitrary. The WKB expression for the wave function in the separated coordinates factorizes into a product of single-particle wave functions

$$
\Psi_{N, q}^{(\mathrm{SoV})}=\mathrm{e}^{i \vec{P} \cdot \vec{b}_{0}} Q\left(\vec{x}_{1}\right) \ldots Q\left(\vec{x}_{N-1}\right)
$$


with $Q\left(\vec{x}_{k}\right) \sim \exp \left(i S_{0}\left(\vec{x}_{k}\right)\right)$. According to (4.30), the momentum, $p_{x}$, and, as a consequence, the action function $S_{0}(\vec{x})$ are multi-valued functions of $x$. Denoting the different branches of the action function by $S_{0, \alpha}(\vec{x})$, one writes the WKB expression for the wave function of the quantum spin magnet as a sum over branches $[17,100]$

$$
Q(\vec{x})=\sum_{\alpha} A_{\alpha}(\vec{x}) \exp \left(\frac{i}{\hbar} S_{0, \alpha}(\vec{x})\right),
$$

with $\hbar=1$. The function $A_{k}(\vec{x})$ takes into account subleading WKB corrections and is fixed uniquely by $S_{0, \alpha}(\vec{x})$. The quantization conditions for the charges $\boldsymbol{q}$ follow from the requirement that (4.34) has to be a single-valued function of $\vec{x}$. As was shown in Refs. $[44,96]$, these conditions can be expressed in terms of the periods of the "action" differential over the canonical set of the $\alpha$ - and $\beta$-cycles on the Riemann surface corresponding to the complex curve 4.30

$$
\Re \mathrm{e} \oint_{\alpha_{k}} d x p_{x}=\pi \ell_{2 k-1}, \quad \Re \mathrm{e} \oint_{\beta_{k}} d x p_{x}=\pi \ell_{2 k},
$$

with $k=1, . ., N-2$ and $\ell=\left(\ell_{1}, \ldots, \ell_{2 N-4}\right)$ being the set of integers. The relations (4.35) define the system of $2(N-2)$ real equations on the $(N-2)$ complex charges $q_{3}, \ldots, q_{N}$ (we recall that the eigenvalues of the "lowest" charge $q_{2}$ are given by (4.25)). Their solution leads to the semiclassical expression for the eigenvalues of the conserved charges. In turn, the energy of the $N$-reggeon states $E_{N, q}$ can be expressed as a function of $q_{3}, \ldots, q_{N}$. In the semiclassical approach, the corresponding expression is

$$
\begin{aligned}
E_{N}^{(\mathrm{as})} & =4 \ln 2 \\
& +2 \Re \mathrm{e} \sum_{k=0}^{N}\left[\psi\left(1+i \Re \mathrm{e} \lambda_{k}+\left|\Im \mathrm{m} \lambda_{k}\right|\right)+\psi\left(i \Re \mathrm{e} \lambda_{k}+\left|\Im \mathrm{m} \lambda_{k}\right|\right)-2 \psi(1)\right]
\end{aligned}
$$

where $\lambda_{k}$ are roots of the polynomial $t_{N}(u)$ (see Eq. (4.31)). The expression in Eq. (4.36) is similar to the energy of the $S L(2, \mathbb{R})$ magnet in Eq. (2.69) although the properties of the two models are different. As was demonstrated in [96], the resulting semiclassical expressions for $q_{3}, \ldots, q_{N}$ and $E_{N}$ are in good agreement with exact results $[27,28]$. As an example, we show in Fig. 6 a comparison of the exact spectrum of the conserved charge $q_{3}$ for the $N=3$ states carrying the conformal spin $h=1 / 2$ (or equivalently $n_{h}=\nu_{h}=0$ ) 


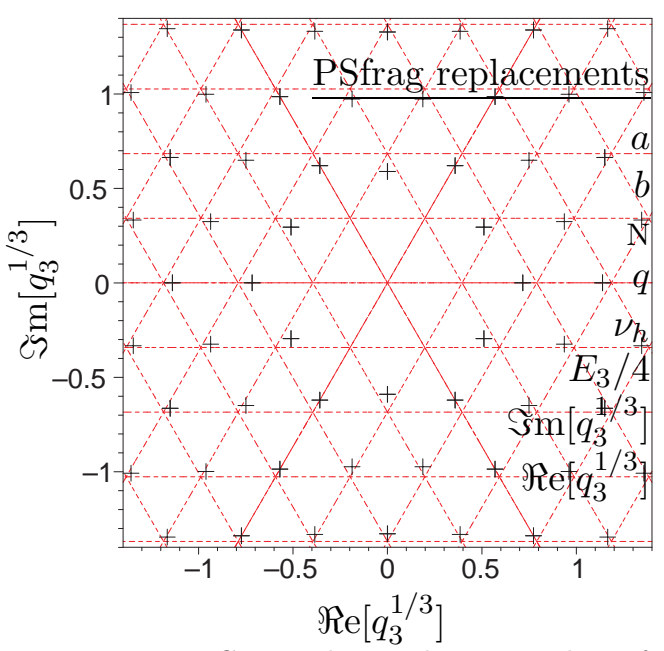

Figure 6. Lattice structure at $N=3$. Crosses denote the exact values of $q_{3}^{1 / 3}$ at $h=1 / 2$. Dotted lines intersect at the points defined in Eq. 4.37.

with the semiclassical expression given by

$$
q_{3}^{1 / 3}=\frac{\Gamma^{3}(2 / 3)}{2 \pi}\left[\frac{1}{2}\left(\ell_{1}+\ell_{2}\right)+i \frac{\sqrt{3}}{2}\left(\ell_{1}-\ell_{2}\right)\right]
$$

with integer $\ell_{1}$ and $\ell_{2}$ introduced in (4.26).

A novel feature of the quantization conditions (4.35) is that they involve both the $\alpha$ - and $\beta$-periods on the Riemann surface. This should be compared with the situation in the $S L(2, \mathbb{R})$ Heisenberg magnet discussed in Section 2.4. There, the WKB quantization conditions involve only the $\alpha$-cycles, Eq. (2.67), since the $\beta$-cycles correspond to classically forbidden zones. For the $S L(2, \mathbb{C})$ magnet, the classical trajectories wrap over an arbitrary closed contour on the spectral curve (4.30) leading to 4.35). This fact allows one to explore the full modular group [101] of the complex curve (4.30) and explain the lattice structure of the spectrum shown in Fig. 6 ] We refer the interested reader to Ref. [96] for details.

\section{Spin chains and $\mathcal{N}=2$ super Yang-Mill theories}

In this section, we shall briefly discuss the integrability phenomenon which emerged from the calculation of the low-energy effective action in $\mathcal{N}=2$ SYM theory in Refs. [29,102]. Rather than delivering an extensive review of the subject, we shall outline the similarities and differences with the cases discussed in the previous sections. Detailed reviews of the solutions to $\mathcal{N}=2$ SYM theories are given in $[103,104]$ while reviews of the integrable struc- 
tures in low-energy effective actions can be found in [105-107]. We will argue below that the low-energy effective action in the $\mathcal{N}=2$ theory with matter in the fundamental representation is governed by integrable models which belong to the same universality class as the spin chains that we encountered in the studies of anomalous dimensions of Wilson operators and high-energy scattering in QCD. The particular form of the model depends on the number of matter hypermultiplets $N_{f}$. To preserve the asymptotic freedom in the $\mathcal{N}=2 \mathrm{SYM}$ theory with the $S U\left(N_{c}\right)$ gauge group, one requires that $N_{f}$ does not exceed $2 N_{c}$. For $N_{f}=2 N_{c}$ the beta-function in the $\mathcal{N}=2$ theory vanishes and the low-energy effective action is described by an inhomogeneous twisted Heisenberg XXX spin chain while for $N_{f}<2 N_{c}$ one encounters its degenerations $[108,109]$.

The classical action of the $\mathcal{N}=2$ SYM theory has the following form

$$
\begin{gathered}
\mathcal{S}=\Im \mathrm{m} \int d^{4} x\left[\tau \int d^{2} \theta d^{2} \bar{\theta} \operatorname{tr}\left(\Phi^{\dagger} e^{V} \Phi+Q_{k}^{\dagger} e^{V} Q^{k}+\tilde{Q}_{k}^{\dagger} e^{V} \tilde{Q}^{k}\right)\right. \\
\left.+\tau \int d^{2} \theta\left(\operatorname{tr} W^{2}+\tilde{Q}_{k} \Phi Q^{k}+m_{k}^{l} \widetilde{Q}_{l} Q^{k}\right)\right]
\end{gathered}
$$

where $\tau$ is the complex coupling constant

$$
\tau=\frac{4 \pi i}{g^{2}}+\frac{\theta}{2 \pi} .
$$

$\Phi$ is the scalar, chiral $\mathcal{N}=1$ superfield involving the scalar fields and fermions. $V$ is the vector $\mathcal{N}=1$ superfield involving the gauge field and its superpartner (the gluino), $W$ is the spinor superfield constructed from $V$ in such a way that $\operatorname{tr} W^{2}$ gives rise to kinetic terms for the gluons and gluinos. The superfields $\widetilde{Q}_{k}$ and $Q_{k}$ (with $k=1, \ldots, N_{f}$ ) represent matter in the fundamental and anti-fundamental representations of the gauge group $S U\left(N_{c}\right)$, respectively, while the superfields $\Phi$ and $W$ belong to the adjoint representation. The $\mathcal{N}=1$ superfields $W$ and $\Phi$ can be combined together into the $\mathcal{N}=2$ chiral superfield $\Psi_{\mathcal{N}=2}$, while pairing $\widetilde{Q}_{k}$ and $Q_{k}$ yields the $\mathcal{N}=2$ hypermultiplet.

In the $\mathcal{N}=2 \mathrm{SYM}$ theory, the vacuum states which do not break supersymmetry are field configurations with vanishing energy. It turns out that there are vacuum valleys, parameterized by the vacuum expectation values of the fields $Q_{k}$ and $\Phi$. We shall be interested in the so-called Coulomb branch where all matter condensates vanish, $\left\langle Q_{k}\right\rangle=0$, and the equation defining the condensate of the scalars reads

$$
\operatorname{tr}\left[\phi, \phi^{\dagger}\right]^{2}=0 .
$$


Up to an $S U\left(N_{c}\right)$ gauge rotation, the expectation values of the scalar field can be chosen to lie in the Cartan subalgebra $\phi=\operatorname{diag}\left(a_{1}, \ldots, a_{N_{c}}\right)$ with $\operatorname{tr} \phi=$ 0 . The parameters $a_{k}$ cannot serve, however, as good order parameters, since there is still a residual Weyl symmetry which changes $a_{k}$ but leaves gauge-invariant quantities intact. An appropriate set of gauge invariant order parameters that fix the vacuum state unambiguously is provided by $u_{k}=\left\langle\operatorname{tr} \phi^{k}\right\rangle$ with $k=2, \ldots, N_{c}$. The variables $u_{2}, \ldots, u_{N_{c}}$ parameterize the space of vacua in the $\mathcal{N}=2$ theory - the moduli space, and the choice of a point on the Coulomb branch is equivalent to a choice of the vacuum state. Simultaneously, the expectation value of the scalars yields the scale at which the coupling constant is frozen. At a generic point on the moduli space, for non-vanishing $\phi$, the $S U\left(N_{c}\right)$ gauge symmetry is broken by the standard Higgs mechanism and hence massive gauge bosons emerge. Integrating them out, one arrives at the well-defined problem of the determination of the lowenergy effective action of the $\mathcal{N}=2$ SYM theory.

To calculate the $\mathcal{N}=2$ low-energy effective action, one has to modify the classical action (5.1) by taking into account one-loop perturbative corrections and, in addition, include instanton corrections. The explicit summation over the instantons is a complicated problem which has been solved only recently [110]. In their original work [29, 102], Seiberg and Witten avoided this problem and calculated the low-energy effective action by making use of three ideas: holomorphicity, duality and their compatibility with renormalization-group flows. Holomorphicity implies that the low-energy effective action depends on a single holomorphic function $\mathcal{F}$ called prepotential

$$
\mathcal{S}_{\text {low-energy }}=\Im \mathrm{m} \int d^{4} x \int d^{4} \theta d^{4} \bar{\theta} \mathcal{F}\left(\Psi_{\mathcal{N}=2}\right) .
$$

Thus, the problem effectively reduces to the determination of the prepotential. The holomorphic function $\mathcal{F}(a)$ is defined unambiguously by its behavior in the vicinity of singular points on the complex $a$-plane. One of the singularities, corresponding to large values of the condensate, is fixed by the perturbative contribution to the $\mathcal{N}=2$ low-energy effective action. The behavior at the remaining singularities is fixed by the use of duality and non-renormalization theorems for the central charges of the SUSY algebra.

To define the duality transformations relating the weak and strong coupling constant regimes in the $\mathcal{N}=2$ SYM theory, it is instructive to examine first the $\mathcal{N}=4$ model. In this model, the coupling constant does not run and the duality transformations are generated by $\tau \rightarrow \tau^{-1}$ and $\tau \rightarrow \tau+1$. The same transformations define the modular group acting on the moduli 
space of some Riemann surface parameterized by $\tau$. This suggests that the duality properties of the SYM theory are encoded in the properties of Riemann surfaces. Going over from $\mathcal{N}=4$ to $\mathcal{N}=2$ theory, one finds that naive generalization of the duality meets serious difficulties. The reason for this is that, in the asymptotically free theory, one has to marry the duality with the renormalization group flow of the coupling constant $\tau$. The solution to this problem is that one is still able to connect the duality and modular transformations if one considers the $\mathcal{N}=2$ theory at different vacua, connected to each other by the duality transformation. Then, the duality acts on the moduli space of vacua which is associated with the moduli space of the auxiliary Riemann surface. At the next step, one has to find out proper variables whose modular properties fit the field theory interpretation. These variables are identified with the integrals of a meromorphic 1-form $d S$ over cycles on some Riemann surface $[29,102]$

$$
a_{i}=\oint_{\alpha_{i}} d S, \quad a_{D, i}=\oint_{\beta_{i}} d S,
$$

where $i=1, \ldots, N_{c}-1$ for the $S U\left(N_{c}\right)$ gauge group.

The integrals (5.5) play a two-fold role in the Seiberg-Witten approach. Firstly, they allow one to determine the prepotential $\mathcal{F}(a)$ through the relation

$$
a_{D, i}(a)=\frac{\partial \mathcal{F}(a)}{\partial a_{i}}
$$

with $a=\left(a_{1}, \ldots, a_{N_{c}-1}\right)$ and, therefore, evaluate the low energy effective action (5.4). Then, using the property of the differential $d S$ that its variations w.r.t. moduli are holomorphic, one can also calculate the matrix of coupling constants

$$
T_{i j}(u)=\frac{\partial^{2} \mathcal{F}(a)}{\partial a_{i} \partial a_{j}}
$$

Secondly, the periods $a$ and $a_{D}$, Eq. (5.5), define the spectrum of stable states in the theory which saturate the Bogomolny-Prasad-Sommerfeld (BPS) bound. For instance, the dyonic spectrum of stable particles of a $S U(2)$ theory reads

$$
M_{n, m}=\left|n a(u)+m a_{D}(u)\right|
$$

with $n$ and $m$ integeral.

The Seiberg-Witten solution admits an elegant interpretation in terms of classical integrable systems. The Riemann surface entering (5.5) defines 
the solution to the classical equations of motion in a classical integrable system. Moreover, the meromorphic differential $d S$ turns out to be the action differential in the separated variables in the same system, $d S=p(x) d x$, with $p(x)$ being a single-valued function on the Riemann surface [111]. The dependence of the prepotential on the fundamental scale $\Lambda$ is given by [112]

$$
\frac{\partial \mathcal{F}(a)}{\partial \ln \Lambda}=\beta u_{2}(a) \equiv \beta H,
$$

where $\beta$ is the one-loop beta-function in the $\mathcal{N}=2 \mathrm{SYM}$ theory. This equation has another interpretation as the evolution equation in an integrable system. $H$ coincides with the Hamiltonian of this system and $\ln \Lambda$ is the evolution time variable. Hence we see, once again, that the logarithm of the relevant scale plays the rôle of time in the integrable dynamics.

As was already mentioned, the Riemann surface parameterizing the Seiberg-Witten solution for the low-energy effective action in the $\mathcal{N}=2$ SYM theory with fundamental matter [108] coincides with the spectral curve describing the solution to the classical equation of motion in the periodic inhomogeneous Heisenberg XXX spin chain of length $N_{c}$ [113,114]. The masses of the squarks in the $\mathcal{N}=2$ theory are mapped into the parameters of the chain while the coordinates on the Coulomb branch of the moduli space are mapped into the values of the integrals of motion. The spectral curve for the spin chain system is defined as

$$
\operatorname{det}\left(w-T_{N_{c}}(x)\right)=0,
$$

where $T_{N_{c}}(x)=\operatorname{tr}\left[L_{1}(x) \ldots L_{N_{c}}(x)\right]$ is the transfer matrix of the model and $L_{i}(x)$ is a local $2 \times 2$ Lax matrix given by

$$
L_{i}(x)=\left(x+\lambda_{i}\right) \cdot \mathbf{1}+\sum_{a=1}^{3} S_{a, i} \cdot \sigma^{a} .
$$

Here $\sigma^{a}$ are the Pauli matrices and $\lambda_{i}$ are the chain inhomogeneities. The Poisson brackets of the dynamical variables $S_{a},(a=1,2,3)$ are just

$$
\left\{S_{a, j}, S_{b, k}\right\}=-i \epsilon_{a b c} S_{c, j} \delta_{j k},
$$

so that the vector $\left\{S_{a}\right\}$ plays the rôle of the angular momentum ("classical spin"). The spectral curve (5.10) is now

$$
w+\frac{Q(x)}{w}=2 P(x)
$$


with $P(x) \equiv \operatorname{tr} T(x) / 2$ and $Q(x) \equiv \operatorname{det} T(x)$. In the hyper-elliptic parameterization, $y=(w+Q(x) / w) / 2$, the same curve becomes

$$
y^{2}=P^{2}(x)-Q(x) .
$$

The zeroes of $Q(x)$ define the masses of the hypermultiplets in the $\mathcal{N}=2$ SYM theory via

$$
Q(x)=\prod_{i=1}^{N_{c}} \operatorname{det} L_{i}(x)=\prod_{i=1}^{N_{c}}\left(x-m_{i}^{+}\right)\left(x-m_{i}^{-}\right)
$$

where $m_{i}^{ \pm}=-\lambda_{i} \pm s_{i}, S_{a, i}^{2}=s_{i}^{2}$ and $s_{i}$ stands for the spins at the $k$ th site of the chain. By construction, the number of matter multiplets is twice the number of colors. Still, Eq. (5.14) is not the most general form of the spectral curve for $N_{f}=2 N_{c}$.

Equation (5.14) can be generalized by taking into account the additional freedom in the definition of the spin chain and the spectral curve. Namely, one can multiply the Lax operator of the spin chain by an arbitrary constant matrix without changing the commutation relations and conservation laws. Moreover, one can also attach a constant (external magnetic field) matrix $V$ to the end of the chain, that is $L_{N_{c}}(x) \rightarrow L_{N_{c}}(x) V$. This amounts to imposing more general (twisted) boundary conditions along the spin chain. The spectral curve corresponding to the "twisted" spin chain takes the form $[102,113,114]$

$$
\begin{aligned}
& w+\frac{Q(x)}{w}=P(x), \\
& P(x)=\prod_{i=1}^{N_{c}}\left(x-\phi_{i}\right), \\
& Q(x)=h(h+1) \prod_{j=1}^{2 N_{c}}\left(x-m_{j}-\frac{2 h}{n} \sum_{i} m_{i}\right) .
\end{aligned}
$$

Let us now consider these formulae in the special case of a superconformal $\mathcal{N}=2$ SYM theory with $N_{f}=2 N_{c}$ massless fundamental hypermultiplets $[102,113-117]$. The corresponding integrable system is described by the spectral curve

$$
y^{2}=P^{2}(x)-4 x^{2 N_{c}}\left(1-\rho^{2}\left(\tau_{c l}\right)\right),
$$

where $\rho^{2}\left(\tau_{c l}\right)$ is some function of the coupling constant in the $\mathcal{N}=2$ theory and the polynomial $P(x)$ depends on the coordinates on the moduli space 


$$
\begin{aligned}
& \vec{u}=\left(u_{2}, \ldots, u_{N_{c}}\right) \\
& P(x)=\sum_{k=0}^{N_{c}} q_{k}(\vec{u}) x^{N_{c}-k}=2 x^{N_{c}}+q_{2} x^{N_{c}-2}+\ldots+q_{N_{c}},
\end{aligned}
$$

where $q_{0}=2, q_{1}=0$ and the other $q_{k}$ are some known functions of $\vec{u}$. Their explicit form is not important for our purposes. The Seiberg-Witten meromorphic differential on the curve is given by

$$
d S=p d x=\ln \left(\omega / x^{N_{c}}\right) d x
$$

where $y=\omega-x^{2 N_{c}} / \omega$ and $\omega=x^{N_{c}} \mathrm{e}^{p}$.

From the point of view of integrable models, the spectral curve (5.17) corresponds to a classical Heisenberg XXX spin chain of length $N_{c}$ with spin-zero at all sites (due to $q_{1}=0$ ) and parameter $\rho$ related to the external magnetic field, or equivalently, to the twisted boundary conditions [109]. Remarkably enough, the spectral curve (5.17) describing the low-energy effective action of the superconformal $\mathcal{N}=2$ SYM theory with $N_{f}=2 N_{c}$ is closely related to the spectral curve (4.30) describing the spectrum of multireggeon compound states in multicolor QCD [44]. It is easy to verify that the two curves coincide if one makes the following identification:

- The number of the reggeons $N=N_{c}$;

- The integrals of motion for the multi-reggeon state are identified as the above-mentioned functions $q_{k}(\vec{u})$ on the moduli space of the superconformal theory;

- The coupling constant of the gauge theory should be such that $\rho\left(\tau_{c l}\right)=$ 0 that is $\tau_{\mathrm{cl}}=\frac{1}{2}+\frac{i}{2} \tan \frac{\pi}{2 N_{c}}$

Under these conditions the two theories fall into the same universality class.

Let us briefly mention some recent developments concerning the relation between integrable systems and $\mathcal{N}=1 \mathrm{SYM}$ theories. In the $\mathcal{N}=2 \mathrm{SYM}$ theory, the Riemann surface degenerates at some points on the Coulomb branch of the moduli space. After soft breaking of $\mathcal{N}=2$ down to $\mathcal{N}=1$, these points correspond to the vacuum states of the $\mathcal{N}=1 \mathrm{SYM}$ theory. Some massless states condense at these points leading to the formation of a mass gap and to confinement. For instance, in the $S U(2)$ case the $\mathcal{N}=1$ vacua correspond to the points $u_{2}= \pm \Lambda^{2}$ where the monopoles or dyons become massless and condense. It turns out that the relation to the integrable systems becomes even more direct after soft breaking. Namely the treelevel superpotential $W_{\text {tree }}=\sum_{k} t_{k} \operatorname{tr} \Phi^{k}$ amounts to the following effective 
superpotential

$$
W_{\mathrm{eff}}=\sum_{k} t_{k} \operatorname{tr} L^{k}
$$

where $L$ is the Lax operator of the corresponding integrable system governing the $\mathcal{N}=2$ theory with the same matter content [118-120]. That is $W_{\text {eff }}=\sum_{k} t_{k} H_{k}$, and vacua in the $\mathcal{N}=1$ theory defined by the extremization of $W_{\text {eff }}$ are in one-to-one correspondence with the equilibrium states of the corresponding integrable system with respect to the combination of Hamiltonians above.

\section{Gauge/string correspondence}

In previous sections, we described the hidden integrability symmetry of the one-loop dilatation operator in QCD and its supersymmetric extensions. Its origin, however, remains obscure on the gauge theory side. In this section we shall describe attempts to explain the integrability phenomena in YangMills theories based on the gauge/string correspondence. We will demonstrate that quantum integrability of the dilatation operator in gauge theory is ultimately related to classical integrability of the relevant stringy sigma models. The subject is rapidly developing but a fully satisfactory picture has not yet emerged.

To start with, let us recall the general features of the gauge/string correspondence relevant for our discussion. In the case of the $\mathcal{N}=4 \mathrm{SYM}$ theory it states that this gauge theory is dual to IIB string theory on an $A d S_{5} \times S^{5}$ background with additional flux of a higher-form field [38,121]. The background metric in Poincaré coordinates has the form

$$
d s^{2}=\frac{r^{2}}{R^{2}}\left(-d t^{2}+d x_{1}^{2}+d x_{2}^{2}+d x_{3}^{2}\right)+R^{2} \frac{d r^{2}}{r^{2}}+R^{2} d \Omega_{5}^{2}
$$

and can be considered as the near-horizon limit of the D3 brane metric. Since the D3-brane is a source of the Ramond-Ramond four-form field $A_{4}$, the background solution is supplemented by the flux of the corresponding field strength

$$
F_{5}=d A_{4}, \quad \int_{S^{5}} * F_{5}=N_{c} .
$$

The radii of the $A d S_{5}$ and $S^{5}$ are identical and equal to

$$
R^{4}=4 \pi g_{s} \alpha^{2} N_{c} .
$$


The four-dimensional gauge theory is localized on the boundary of the $A d S_{5}$. The conformal $S O(2,4)$ group and the $R$-symmetry $S O(6)$ group of the $\mathcal{N}=4 \mathrm{SYM}$ theory are identified with the isometry group of the $A d S_{5}$ and $S^{5}$ spaces, respectively. According to the gauge/string duality, the eigenvalues of the dilatation operator in the $\mathcal{N}=4 \mathrm{SYM}$ theory and the energy spectrum of the string in radial quantization coincide.

The string tension is related to the gauge theory 't Hooft coupling constant by

$$
T=\frac{\sqrt{\lambda}}{2 \pi}=\frac{R^{2}}{2 \pi \alpha^{\prime}}, \quad \lambda=g^{2} N_{c} .
$$

Therefore the strong coupling regime $\lambda \gg 1$ in the gauge theory corresponds to the semiclassical regime on the stringy side. In this way, the classical string calculations provide predictions for many interesting quantities in the $\mathcal{N}=4$ SYM theory (like anomalous dimensions, condensates, Wilson loops etc.) in the strong coupling regime [121]. However, these predictions can be checked diagrammatically only in a few examples, such as for a circular Wilson loop $[122,123]$. On the other hand, to approach the weak coupling regime in Yang-Mills theory, one should go beyond the semiclassical approximation and take into account quantum effects on the string theory side.

At present, an explicit quantum solution of the string theory in the $A d S_{5} \times S^{5}$ background is not available. This makes it impossible to compare the string spectrum with the complete set of operators in $\mathcal{N}=4$ SYM theory. Hopefully the explicit solution of the string theory can be found [124] in the case of the limiting geometry defined as the Penrose limit of the $A d S_{5} \times S^{5}[39,125,126]$. The Penrose limit can be described as the region around the null geodesic in the $A d S_{5} \times S^{5}$. Introducing new variables

$$
x^{+}=\frac{t+\chi}{2 \mu}, \quad x^{-}=\mu R^{2}(t-\chi)
$$

with $\chi$ being an angular variable in $S^{5}$ and $\mu$ being some scale, one takes the limit $R \rightarrow \infty$ and recovers the pp-wave metric

$$
d s^{2}=-4 d x^{+} d x^{-}-z^{2} d x^{+2}+\sum_{i=1}^{8} d z_{i}^{2} .
$$

Here, eight, flat, transverse coordinates $z_{i}$ come both from the $S^{5}$ and $A d S_{5}$ parts of the geometry. In this metric, the string behaves as a particle rotating with large angular momentum $J$ along the angular coordinate $\chi$ in $S^{5}$. The 
light-cone energy of the string is

$$
H=2 p^{-}=i\left(\partial_{t}+\partial_{\chi}\right)=(\Delta-J) .
$$

For $R \rightarrow \infty$ it takes finite values provided that the following double scaling limit is considered:

$$
R \rightarrow \infty, \quad \Delta \sim J \rightarrow \infty, \quad \frac{J^{2}}{R^{4}}=\text { const } .
$$

Quantization of the string propagating in this background reduces to the quantization of the oscillators. As a result, the exact spectrum of the type IIB string in the pp-wave background looks like

$$
\Delta-J=\sum_{k} N_{k} \sqrt{1+\frac{\lambda k^{2}}{J^{2}}},
$$

where $k$ labels the Fourier modes, $N_{k}$ denotes the total occupation number of oscillatory mode and the condition $P=\sum_{k} k N_{k}=0$ is imposed.

On the gauge theory side, (6.9) defines the anomalous dimensions of certain Wilson operators in the $\mathcal{N}=4$ SYM theory. The length of the string $J$ equals the number of constituents of the composite operator. The ground state of the string can be identified with the operator built from scalars $Z=\Phi_{1}+i \Phi_{2}$

$$
|0, J\rangle \leftrightarrow \operatorname{tr} Z^{J}
$$

These operators have charge $J$ with respect to the "rotation plane" in the pp-wave. The oscillatory excitations of the string ground state correspond to insertions of other scalar fields. For example, the so-called BMN operators in the $\mathcal{N}=4 \mathrm{SYM}$ theory can be mapped into the stringy modes as follows

$$
\begin{aligned}
a_{0}^{i+}|0, J\rangle & \Leftrightarrow \operatorname{tr} \Phi_{i} Z^{J}, \\
a_{n}^{i+} a_{-n}^{j+}|0, J\rangle & \Leftrightarrow \sum_{l} e^{2 \pi i n l / J} \operatorname{tr} \Phi_{i} Z^{l} \Phi_{j} Z^{J-l} .
\end{aligned}
$$

One can deduce from these expressions that calculation of the stringy spectrum corresponds to diagonalization of the mixing matrix for Wilson operators on the gauge theory side.

The energy of the string in the pp-wave limit is a function of the ratio of the coupling constant and angular momentum, $\lambda / J^{2}$. It is expected that expansion of this function in powers of $\lambda / J^{2}$ should reproduce a perturbative series for the anomalous dimension of the corresponding Wilson operators in the weak-coupling regime. On the other hand, the one-loop $\mathcal{N}=4$ dilatation 
operator in the sector of scalar operators coincides with the Hamiltonian of the Heisenberg $S O(6)$ spin chain [25]. This allows one to map stringy states into spin chain states. The correspondence is very precise for Wilson operators built only from two complex scalars, in which case the $S O(6)$ spin chain reduces to the conventional Heisenberg $S U(2)$ spin $-1 / 2$ chain. Then, the ground state in the string theory corresponds to all spins aligned in the same direction in the isotopic space while the stringy excitations correspond to flipping some spins along the chain.

The BMN operators are the only well-established example in which the exact quantum answer on the stringy side can be matched into the all-loop anomalous dimension on the gauge theory side. Later in this section, we shall discuss generalized BMN operators which can be treated semiclassically on the stringy side and demonstrate that the corresponding solutions to the classical equations of motion in the stringy sigma models are ultimately related to classical integrable models. We shall explain the relation between the string sigma model and quantum spin chains in the thermodynamical limit and demonstrate that the anomalous dimensions of the certain operators are in one-to-one correspondence with the special class of classical solutions to the sigma model for which this model reduces to finite dimensional integrable systems of the Neumann type. Finally, we shall comment on the relation between general classical solutions to the sigma model and the Bethe Ansatz solution to the compact quantum spin chains in the semiclassical limit.

\subsection{Derivation of the string in the thermodynamical limit}

The sigma model describing the string moving in the appropriate curved background can be derived from the quantum compact spin chain in the long-wavelength limit. The corrections to the classical sigma model scale as $1 / J$, where the angular momentum of the string $J$ corresponds on the gauge theory side to the number of fields entering the composite operator or equivalently the length of the spin chain. The transition from the spin chains to the sigma model relies on the coherent state formalism [42].

Let $|s s\rangle$ be the state with total spin $s$ and projection onto the $z$-axis $S_{z}=s$. The coherent state for the spin-s representation of the $S U(2)$ group is defined as

$$
|\vec{n}\rangle=\mathrm{e}^{i S_{x} \phi} \mathrm{e}^{i S_{y} \theta}|s s\rangle
$$

where $\vec{n}$ is the unit vector, $\vec{n}^{2}=1$,

$$
\vec{n}=(\sin \theta \cos \phi, \sin \theta \sin \phi, \cos \theta)
$$


with $\theta$ and $\phi$ being spherical angles. Expanding the Hamiltonian of the spin chain $H=\lambda /\left(4 \pi^{2}\right) \sum_{k=1}^{J}\left(1 / 4-\vec{S}_{k} \cdot \vec{S}_{k+1}\right)$ over the coherent states, one rewrites the partition function $\operatorname{tr} \mathrm{e}^{-H t}$ in the standard manner as a path integral over $\vec{S}_{k}=s \vec{n}_{k}$ with the following action

$$
\mathcal{S}(\vec{n})=s \sum_{k=1}^{J} \int d t \int_{0}^{1} d \tau \vec{n}_{k}\left(\partial_{t} \vec{n}_{k} \times \partial_{\tau} \vec{n}_{k}\right)-\frac{\lambda}{8 \pi^{2}} s^{2} \int d t \sum_{k=1}^{J}\left(\vec{n}_{k}-\vec{n}_{k+1}\right)^{2},
$$

with $\vec{n}_{J+1}=\vec{n}_{1}$. In the long-wavelength limit, the vectors $\vec{n}_{k}(t)$ vary smoothly along the spin chain and, therefore, they can be approximated by a function $\vec{n}(\sigma, t)$ with continuous $\sigma$ running between 0 and the chain length $J$, leading to

$$
\mathcal{S}=-s \int d t d \sigma \partial_{t} \phi \cos \theta-\frac{\lambda}{8 \pi^{2}} s^{2} \int d t d \sigma\left[\left(\partial_{\sigma} \theta\right)^{2}+\left(\partial_{\sigma} \phi\right)^{2} \sin ^{2} \theta\right] .
$$

It turns out [42] that for $s=1 / 2$, this expression coincides with the stringy action

$$
\mathcal{S}_{\mathrm{str}}=\frac{R^{2}}{4 \pi \alpha^{\prime}} \int d \sigma d \tau\left[G_{\mu \nu} \partial_{\tau} X^{\mu} \partial_{\tau} X^{\nu}-G_{\mu \nu} \partial_{\sigma} X^{\mu} \partial_{\sigma} X^{\nu}\right]
$$

evaluated for the classical string propagating in the background $d s^{2}=$ $G_{\mu \nu} d X^{\mu} d X^{\nu}$

$$
d s^{2}=-d t^{2}+d \psi^{2}+d \varphi_{1}^{2}+d \varphi_{2}^{2}+2 \cos (2 \psi) d \varphi_{1} d \varphi_{2} .
$$

To see this, one fixes the gauge $t=\chi \tau$, takes the limit $\partial_{\tau} X^{i} \rightarrow 0$ and $\chi \rightarrow \infty$ with $\chi \partial_{\tau} X^{i}=$ fixed and identifies the variables as

$$
\varphi_{2}=-\frac{1}{2} \phi, \quad \psi=\frac{1}{2} \theta .
$$

Then one eliminates $\varphi_{1}$ with the help of the classical equations of motion and arrives at (6.15).

The derivation of the effective action can be also generalized to the $S U(3)$ case [127] and to the string carrying both large Lorentz spin $S$ and $R$-charge $J$ [128]. One can improve the effective sigma model action (6.15) by calculating corrections involving higher derivatives of the fields. All such terms containing up to four derivatives have been found in Refs. [129,130].

\subsection{Semiclassical string motion and integrable models}

We have argued above that the effective action for long-wavelength excitations in the compact spin chain coincides with the classical action of the 
sigma model on the curved background relevant for calculation of the anomalous dimensions of BMN-like operators. As the next step, the corresponding solutions to the equations of motion are compared. To this end, one considers the bosonic part of the superstring action on the $A d S_{5} \times S^{5}$ background. It is given by the sum of two coset sigma models

$$
\mathcal{S}=\frac{\sqrt{\lambda}}{4 \pi} \int d \sigma d \tau\left[G_{m n}^{A d S} \partial y_{m} \partial y_{n}+G_{k l}^{S^{5}} \partial x^{k} \partial x^{l}\right],
$$

where the string tension is proportional to the 't Hooft coupling, Eq. (6.4). It is convenient to rewrite the action with the constraint imposed by the Lagrangian multiplier

$$
\mathcal{S}=\frac{\sqrt{\lambda}}{4 \pi} \int d \sigma d \tau\left[\partial X_{m} \partial X_{m}+\Lambda_{x}\left(X^{2}-1\right)+\partial Y^{k} \partial Y^{k}+\Lambda_{y}\left(Y^{2}+1\right)\right]
$$

where $X_{n}(n=1, \ldots 6)$ and $Y_{k}(k=0, \ldots, 5)$ are the two sets of the embedded coordinates in the flat $R^{6}$ space with signatures $(6,0)$ and $(4,2)$, respectively. The action has to be supplemented by the Virasoro constraint for the vanishing of the two-dimensional energy momentum tensor

$$
\dot{Y}_{k} \dot{Y}_{l}+Y_{k}^{\prime} Y_{l}^{\prime}+\dot{X}_{n} \dot{X}_{n}+X_{n}^{\prime} X_{n}^{\prime}=\dot{Y}_{k} Y_{k}^{\prime}+\dot{X}_{n} X_{n}^{\prime}=0
$$

and by the periodic boundary conditions

$$
Y_{k}(\sigma+2 \pi)=Y_{k}(\sigma), \quad X_{n}(\sigma+2 \pi)=X_{n}(\sigma) .
$$

Due to the $S O(2,4)$ and $S O(6)$ symmetries, the classical action possesses the set of conserved charges

$$
\begin{aligned}
S_{k l} & =\sqrt{\lambda} \int d \sigma\left(Y_{k} \dot{Y}_{l}-Y_{l} \dot{Y}_{k}\right), \\
J_{n m} & =\sqrt{\lambda} \int d \sigma\left(X_{n} \dot{X}_{m}-X_{m} \dot{X}_{l}\right) .
\end{aligned}
$$

Among them, one distinguishes 6 Cartan generators: the energy $E=S_{05}$, the Lorentz spins $S_{12}, S_{34}$ and the $S^{5}$ angular momenta $J_{12}, J_{34}, J_{56}$. These conserved charges parameterize general solutions to the classical equations of motion [131].

To describe a particular operator on the gauge theory side, we have to identify the corresponding solution to the classical equations of motion in the sigma model (6.20), subject to the constraints (6.21) and (6.22). The simplest ansatz, corresponding to a string located at the center of the $A d S_{5}$ and rotating in the $S^{5}$, is

$$
Y_{5}+i Y_{0}=\mathrm{e}^{i t}, \quad X_{2 i-1}+i X_{2 i}=r_{i}(\sigma) \mathrm{e}^{i \omega_{i} \tau+i \alpha_{i}(\sigma)} .
$$


with $i=1,2,3$ and the remaining $Y$-coordinates set to zero. Substituting into the sigma model action (6.20) yields the Lagrangian [132]

$$
\mathcal{L}=\sum_{i=1}^{3}\left(r_{i}^{\prime 2}+r_{i}^{2} \alpha_{i}^{\prime 2}-\omega_{i}^{2} r_{i}^{2}\right)-\Lambda_{x} \sum_{i=1}^{3}\left(r_{i}^{2}-1\right) .
$$

Solving the equations of motion for $\alpha_{i}$ one gets $\alpha_{i}^{\prime}=v_{i} / r_{i}^{2}$ with $v_{i}$ being the integration constants. The resulting Lagrangian describes the integrable Neumann-Rosochatius system. ${ }^{1}$ It admits five independent integrals of motion: $v_{1}, v_{2}, v_{3}$ plus two additional integrals of the form

$$
I_{i}=r_{i}^{2}+\sum_{j \neq i}^{3} \frac{1}{\omega_{i}^{2}-\omega_{j}^{2}}\left[\left(r_{i} r_{j}^{\prime}-r_{i} r_{j}^{\prime}\right)^{2}+\frac{v_{i}^{2} r_{j}^{2}}{r_{i}^{2}}+\frac{v_{j}^{2} r_{i}^{2}}{r_{j}^{2}}\right]
$$

subject to $\sum_{i=1}^{3} I_{i}=0$. The periodicity condition trades $v_{i}$ for three integers $m_{i}$ and $I_{i}$ for two integers $n_{i}$. As a result, the energy depends on the frequencies $\omega_{i}$ and five integers. These variables are not independent since the Virasoro constraint imposes a relation between them. For this type of string motion, the infinite set of conserved charges in the sigma model is parameterized by a finite set of integrals of the motion [133,134]. The energy corresponding to classical solutions of the string sigma model defines the anomalous dimension of the dual composite scalar operators in the $\mathcal{N}=4$ SYM theory. There are many examples of such correspondences discussed in the literature, initiated in Ref. [131] and further developed in Refs. [135-140].

It is interesting to note that the Neumann system is isomorphic to the stationary solutions of the Landau-Lifshitz equation. We recall that the time variable in the Neumann system is identified with the coordinate along the string. This identification survives time discretization in which case the Neumann system is equivalent to the discretized version of the LandauLifshitz equation describing the XYZ spin chain [141] and it can be used for the formulation of the integrability in the context of the string bit model [142].

In the above example, the string was rotating in the $S^{5}$. In general, it moves both in the $A d S_{5}$ and $S^{5}$ and could have large angular momenta in both spaces. Contrary to the situation with pure scalar operators, when the comparison with the loop expansion on the gauge side can be performed for operators with large $R$ charge, the situation with operators carrying large Lorentz spin $S$ is more subtle. The folded, closed string rotating in the $A d S_{5}$

\footnotetext{
${ }^{1}$ For vanishing $v_{i}$ the system 6.25 reduces to the Neumann model with three degrees of freedom.
} 
yields the dependence for the anomalous dimensions of twist-two operators $F_{+\perp}\left(D_{+}\right)^{S} F_{+\perp}$ at large coupling of the form [40]

$$
\gamma_{S}^{(\mathrm{tw}=2)}=\frac{\sqrt{\lambda}}{2 \pi} \ln S^{2}
$$

This result can be generalized to higher twist operators of the form $F_{+\perp} D_{+}^{S_{1}} F_{+\perp} \ldots D_{+}^{S_{L-1}} F_{+\perp}$. The energy of the corresponding revolving string coincides with the energy of the classical Heisenberg spin chain of length $L$ and leads to [143]

$$
\gamma_{S_{1}, S_{2}, \ldots, S_{L-1}}^{(\mathrm{tw}=L)}=\frac{\sqrt{\lambda}}{2 \pi} \ln q_{L}\left(S_{1}, S_{2}, \ldots, S_{L-1}\right) .
$$

Here $q_{L}$ is the highest integral of motion of the spin chain. For $S_{k} \sim S \gg 1$ with $k=1, \ldots, L-1$ one has $q_{L} \sim S^{L}$.

Notice that the logarithmic scaling of the anomalous dimensions is a universal feature of Wilson operators with large Lorentz spin in gauge theories, unrelated to the presence of supersymmetry [144,145]. However stringy description of this scaling at weak coupling remains unknown and it is doubtful whether such classical string sigma model solutions exist [140]. One should expect instead that $\sim \ln S$ behavior at weak coupling is driven by the quantum sigma model.

The integrability phenomenon offers the possibility of extending the gauge/string duality beyond the special class of classical string solutions described above. Namely, instead of comparing particular solutions one can identify the integrable structures corresponding to quantum spin chains describing the dilatation operator on the gauge theory side and to classical equations of motion on the string theory side [146]. It turns out that, in both cases, integrability is encoded in the properties of Riemann surfaces.

For the quantum spin chains, the appearance of Riemann surfaces within the framework of the Bethe Ansatz is not surprising. As we already explained in Sect. 2.4, semiclassical solutions to the Baxter equation are determined by the properties of the spectral curve whose genus is proportional to the number of sites in the chain. In Sect. 2 we discussed baryon operators built from three quarks and the corresponding Riemann surface, Eq. (2.66), had genus equal to 1 . In the case of the BMN-like operators, the number of constituent scalar fields goes to infinity in the thermodynamic limit and, therefore, the corresponding Riemann surface would have an infinite genus. However, by choosing the appropriate values of the integrals of motion, it is possible to pinch almost all handles and obtain a finite genus surface. It is this degenerate surface which parameterizes general solutions to the 
classical equations of motion of the string $[146,147]$. The agreement between semiclassical solutions to the Bethe Ansatz equations and solutions to the classical string equations of motion has been carefully checked up to the two-loop level [146].

Although the correspondence between one- and two-loop ${ }^{\mathrm{m}}$ dilatation operators in the $\mathcal{N}=4$ SYM theory Yang-Mills theory, stringy states and integrable quantum spin chains is well established, the situation with higher loops in perturbation theory is unclear. Several proposals have been made concerning integrable structures behind a higher loop dilatation operator $[148,149]$. At the same time, starting at three-loop order the discrepancy seems to arise between expressions for the anomalous dimensions of composite scalar operators with large- $R$ charge and the energy spectrum of the string [150]. More work is needed to clarify this issue.

\subsection{Open string picture for anomalous dimensions}

There exists an alternative description of the logarithmic growth of the anomalous dimensions of Wilson operators with large Lorentz spin, Eq. (6.27) and (6.28), in terms of Wilson loops in the gauge theory and open strings on the $A d S_{5}$ background. This picture relies on the correspondence between the anomalous dimensions of the composite operators with large number of light-cone derivatives and the so-called cusp anomaly of Wilson loops $[144,145]$. It was shown a long time ago [151] that the Wilson loop $W[C]=\operatorname{tr}\left\{P \exp \left(i g \int_{C} d x^{\mu} A_{\mu}(x)\right)\right\}$ acquires a nontrivial anomalous dimension $\Gamma_{\text {cusp }}(\lambda, \theta)$ if the integration contour $C$ has a cusp

$$
\langle W[C]\rangle \sim \mu^{\Gamma_{\text {cusp }}(\lambda, \theta)},
$$

with $\mu$ being a UV cut-off. The cusp angle $\theta$ is restricted to the interval $[0,2 \pi$ [ in Euclidean space but is unrestricted in Minkowski space. The correspondence between the anomalous dimension of twist-2 spin operators with large Lorentz spin $S$ and the cusp anomaly is follows [144,145]

$$
\gamma_{S}^{(\mathrm{tw}=2)}(\lambda)=2 \Gamma_{\text {cusp }}(\lambda, \theta=\ln S)
$$

and is valid for an arbitrary coupling constant $\lambda$. At weak coupling and $\theta \gg 1$, one has

$$
\Gamma_{\text {cusp }}(\lambda, \theta)=\theta\left[\frac{\lambda}{4 \pi^{2}}+\mathcal{O}\left(\lambda^{2}\right)\right]
$$

\footnotetext{
m In a closed subsector only.
} 
with perturbative coefficients known up to three-loop order [152]. The calculation of $\Gamma_{\text {cusp }}(\lambda, \theta)$ at the strong coupling can be effectively done via the open string picture. In this limit, the Wilson loop is proportional to the area of the minimal surface swept out by an open string which penetrates into the fifth AdS dimension and whose ends trace the integration contour $C$ in Minkowski space [122]. This leads to $[153,154]$

$$
\Gamma_{\text {cusp }}(\lambda, \theta)=\theta\left[\left(\frac{\lambda}{4 \pi^{2}}\right)^{1 / 2}+\mathcal{O}\left(\lambda^{0}\right)\right]
$$

for $\theta \gg 1$. Being combined together, Eqs. (6.30) and (6.32) reproduce the strong coupling result (6.27) based on the folded closed string picture [40].

The correspondence (6.30) can be generalized to higher twist operators. In that case, the anomalous dimension of the Wilson operator built from $L$ constituent fields and a total number of derivatives $S$, such that $S \gg L$, can be mapped into the anomalous dimension of the product of $L$ Wilson loops in the fundamental representation of the $S U\left(N_{c}\right)$ group; the total number of cusps varies between 4 and $2 L$ [143]. At large $N_{c}$, the expectation value of the product of Wilson loops factorizes into the product of their expectation values. This implies that, at strong coupling the area of the minimal surface corresponding to the product of $k=2, \ldots, L$ Wilson loops with cusps is given by the sum of $k$ elementary areas leading to

$$
2 \Gamma_{\text {cusp }}(\lambda, \theta=\ln S) \leq \gamma_{S}^{(\mathrm{tw}=L)}(\lambda) \leq L \Gamma_{\text {cusp }}(\lambda, \theta=\ln S) .
$$

We are reminded that the anomalous dimensions of higher twist operators are not solely determined by the total number of derivatives $S$. They form instead a band whose internal structure at weak coupling is governed by integrals of the motion of the quantum Heisenberg $S L(2)$ magnet. Equation (6.33) defines the boundaries of the band both at strong and weak coupling.

\section{Conclusion}

The main objective of the present work was to demonstrate how the phenomenon of integrability arises in certain limiting cases of Yang-Mills dynamics and exhibit their similarities or ultimate relation whenever it was obvious. In all cases we have studied, integrability appears as a hidden symmetry of an underlying effective theory. It either describes elementary fields living on the light cone, in the case of renormalization group evolution in QCD and its supersymmetric extensions, or reggeons as new degrees of freedom in high-energy QCD, or branes as effective degrees of freedom for the Seiberg-Witten solution to the $\mathcal{N}=2$ SYM theory. A number of nontrivial 
questions still have to be answered. The most obvious and at the same time, the most profound and most difficult question is "What is the origin of integrability?" or, in other words, "What is the symmetry, if any, of the gauge theory which leads to it?" More specific questions that have recently kept theoretical physicists busy concern issues like the fate of the integrability of the dilatation operator in SYM at higher orders of perturbation theory, the integrability of the SYM dual sigma models on curved backgrounds and the matching of integrable structures on both sides of the correspondence, to name but a few. Ultimately, if the integrability is indeed a property of the full quantum Yang-Mills theory as well as the dual string theory, it will provide the most sophisticated test of duality and endow us with powerful machinery to tackle the strong-coupling regime of field theories. On this quest, unfortunately, we will be missing Ian Kogan.

\section{Acknowledgements}

Three of us (A.B., V.B and G.K.) are most grateful to Sergei Derkachov and Alexander Manashov for enjoyful and fruitful collaboration on the topics covered in the present work. A.G. would also like to thank A. Marshakov, A. Mironov, A. Morozov, A. Tseytlin and K. Zarembo for the collaboration and discussions of various aspects of integrability and related issues. This work supported (A.B.) in part by U.S. Department of Energy under grant no. DE-FG02-93ER-40762. 


\section{References}

1. K.G. Wilson, Phys. Rev. D 10, 2445 (1974).

2. A.M. Polyakov, Phys. Lett. B 72, 224 (1977); Phys. Lett. B 82, 247 (1979).

3. P.P. Kulish, N.Yu. Reshetikhin and E.K. Sklyanin, Lett. Math. Phys. 5, 393 (1981).

4. V.O. Tarasov, L.A. Takhtajan and L.D. Faddeev, Theor. Math. Phys. 57, 1059 (1983).

5. D.J. Gross and F. Wilczek, Phys. Rev. D 8, 3633 (1973);

H. Georgi and H.D. Politzer, Phys. Rev. D 9, 416 (1974).

6. E.A. Kuraev, L.N. Lipatov and V.S. Fadin, Sov. Phys. JETP 44, 443 (1976); Sov. Phys. JETP 45, 199 (1977);

I.I. Balitsky and L.N. Lipatov, Sov. J. Nucl. Phys. 28, 822 (1978).

7. L.N. Lipatov, JETP Lett. 59, 596 (1994) hep-th/9311037.

8. L.D. Faddeev and G.P. Korchemsky, Phys. Lett. B 342, 311 (1995) hep-th/9404173.

9. G.P. Korchemsky, Nucl. Phys. B 443, 255 (1995) hep-ph/9501232 .

10. V.M. Braun, S.E. Derkachov and A.N. Manashov, Phys. Rev. Lett. 81, 2020 (1998) hep-ph/9805225.

11. A. Gorsky, I.M. Krichever, A. Marshakov, A. Mironov, A. Morozov, Phys. Lett. B 355, 466 (1995) hep-th/9505035.

12. A.P. Bukhvostov, G.V. Frolov, L.N. Lipatov and E.A. Kuraev, Nucl. Phys. B 258, 601 (1985).

13. A.V. Belitsky, Nucl. Phys. B 574, 407 (2000) hep-ph/9907420.

14. S.E. Derkachov, G.P. Korchemsky and A.N. Manashov, Nucl. Phys. B 566, 203 (2000) hep-ph/9909539.

15. Y.M. Makeenko, Sov. J. Nucl. Phys. 33, 440 (1981).

16. V.M. Braun, G.P. Korchemsky and D. Müller, Prog. Part. Nucl. Phys. 51, 311 (2003) hep-ph/0306057.

17. G.P. Korchemsky, Nucl. Phys. B 462, 333 (1996) hep-th/9508025;

Nucl. Phys. B 498, 68 (1997) hep-th/9609123.

18. V.M. Braun, S.E. Derkachov, G.P. Korchemsky and A.N. Manashov, Nucl. Phys. B 553, 355 (1999) hep-ph/9902375.

19. A.V. Belitsky, Phys. Lett. B 453, 59 (1999) hep-ph/9902361.

20. A.V. Belitsky, Nucl. Phys. B 558, 259 (1999) hep-ph/9903512.

21. V.M. Braun, G.P. Korchemsky and A.N. Manashov, Phys. Lett. B 476, 455 (2000) hep-ph/0001130.

22. V.M. Braun, G.P. Korchemsky and A.N. Manashov, Nucl. Phys. B 597, 370 (2001) hep-ph/0010128 ; Nucl. Phys. B 603, 69 (2001) hep-ph/0102313.

23. N. Beisert, Nucl. Phys. B 676, 3 (2004) hep-th/0307015;

N. Beisert and M. Staudacher, Nucl. Phys. B 670, 439 (2003) hep-th/0307042.

24. A.V. Belitsky, S.E. Derkachov, G.P. Korchemsky and A.N. Manashov, Phys. Lett. B 594, 385 (2004) hep-th/0403085; hep-th/0311104

25. J.A. Minahan and K. Zarembo, JHEP 0303, 013 (2003) hep-th/0212208.

26. N. Beisert, C. Kristjansen and M. Staudacher, Nucl. Phys. B 664, 131 (2003) hep-th/0303060.

27. G.P. Korchemsky, J. Kotanski and A.N. Manashov, Phys. Rev. Lett. 88, 122002 (2002) hep-ph/0111185.

28. S.E. Derkachov, G.P. Korchemsky, J. Kotanski and A.N. Manashov, Nucl. Phys. B 645, 237 (2002) hep-th/0204124.

29. N. Seiberg and E. Witten, Nucl. Phys. B 426, 19 (1994) hep-th/9407087. 
30. A. Gorsky, A. Marshakov, A. Mironov and A. Morozov, Phys. Lett. B 380, 75 (1996) hep-th/9603140.

31. A. Gorsky, S. Gukov and A. Mironov, Nucl. Phys. B 517, 409 (1998) hep-th/9707120.

32. L.A. Takhtajan and L.D. Faddeev, Russ. Math. Survey 34, 11 (1979); E.K. Sklyanin, L.A. Takhtajan and L.D. Faddeev, Theor. Math. Phys. 40, 688 (1980); V.E. Korepin, N.M. Bogoliubov and A.G. Izergin, Quantum inverse scattering method and correlation functions, (Cambridge Univ. Press, 1993).

33. I. Bena, J. Polchinski and R. Roiban, Phys. Rev. D 69 046002, (2004) hep-th/0305116.

34. L. Dolan, C.R. Nappi and E. Witten, JHEP 0310, 017 (2003) hep-th/0308089; Yangian symmetry in $D=4$ superconformal Yang-Mills theory, hep-th/0401243

35. L.F. Alday, JHEP 0312, 033 (2003) hep-th/0310146.

36. A.M. Polyakov, Phys. Atom. Nucl. 64, 540 (2001) hep-th/0006132. Int. J. Mod. Phys. A 17S1, 119 (2002) hep-th/0110196; Mod. Phys. Lett. A 19, 1649 (2004) hep-th/0405106.

37. A. Mikhailov, Int. J. Mod. Phys. A 13, 3215 (1998) hep-th/9701174.

38. J.M. Maldacena, Adv. Theor. Math. Phys. 2, 231 (1998) hep-th/9711200;

S.S. Gubser, I.R. Klebanov, A.M. Polyakov, Phys. Lett. B 428, 105 (1998) hep-th/9802109;

E. Witten, Adv. Theor. Math. Phys. 2, 253 (1998) hep-th/9802150.

39. D. Berenstein, J.M. Maldacena and H. Nastase, JHEP 0204, 013 (2002) hep-th/0202021.

40. S.S. Gubser, I.R. Klebanov and A.M. Polyakov, Nucl. Phys. B 636, 99 (2002) hep-th/0204051.

41. G. Arutyunov, S. Frolov, J. Russo and A.A. Tseytlin, Nucl. Phys. B 671, 3 (2003) hep-th/0307191;

N. Beisert, S. Frolov, M. Staudacher and A. A. Tseytlin, JHEP 0310, 037 (2003) hep-th/0308117.

42. M. Kruczenski, Spin chains and string theory, hep-th/0311203

43. A. Klemm, W. Lerche, P. Mayr, C. Vafa and N. P. Warner, Nucl. Phys. B 477, 746 (1996) hep-th/9604034.

44. A. Gorsky, I.I. Kogan and G.P. Korchemsky, JHEP 0205, 053 (2002) hep-th/0204183.

45. S. Mandelstam, Nucl. Phys. B 213, 149 (1983); Phys. Lett. B 121, 30 (1983).

46. L. Brink, O. Lindgren, B.E. Nilsson, Nucl. Phys. B 212, 401 (1983); Phys. Lett. B 123, 323 (1983).

47. S.A. Anikin and O.I. Zavyalov, Annals Phys. 116, 135 (1978).

48. I.I. Balitsky and V.M. Braun, Nucl. Phys. B 311, 541 (1989).

49. D. Müller, D. Robaschik, B. Geyer, F. M. Dittes and J. Horejsi, Phys. Lett. B 209, 325 (1988); Fortsch. Phys. 42, 101 (1994) hep-ph/9812448.

50. G.P. Lepage and S.J. Brodsky, Phys. Rev. Lett. 43, 545 (1979); (E) Phys. Rev. Lett. 43, 1625 (1979).

51. M.E. Peskin, Phys. Lett. B 88, 128 (1979).

52. T. Ohrndorf, Nucl. Phys. B 198, 26 (1982).

53. S.E. Derkachov, J. Phys. A: Math. Gen. 32, 5299 (1999).

54. E.K. Sklyanin, The quantum Toda chain, in Lecture Notes in Physics, vol. 226, Springer, 1985, pp. 196-233; Functional Bethe ansatz, in Integrable and superintegrable systems, ed. B.A. Kupershmidt, (World Scientific, 1990), pp. 8-33; Progr. Theor. Phys. Suppl. 118, 35 (1995) solv-int/9504001. 
55. S.E. Derkachov, G.P. Korchemsky and A.N. Manashov, JHEP 0307, 047 (2003) hep-th/0210216.

56. E.V. Shuryak and A.I. Vainshtein, Nucl. Phys. B 199, 451 (1982); Nucl. Phys. B 201, 141 (1982).

57. A. Ali, V.M. Braun and G. Hiller, Phys. Lett. B 266, 117 (1991).

58. I.I. Balitsky, V.M. Braun, Y. Koike and K. Tanaka, Phys. Rev. Lett. 77, 3078 (1996) hep-ph/9605439.

59. A.V. Belitsky, Phys. Lett. B 405, 312 (1997) hep-ph/9702356.

60. A.V. Belitsky, Leading order analysis of the twist-three spacelike and timelike cut vertices in QCD, in Proc. of the 31st PNPI Winter School on Nuclear and Particle Physics, ed. V.A. Gordeev (St. Petersburg, 1997) p. 369 hep-ph/9703432.

61. G. Ferretti, R. Heise and K. Zarembo, New integrable structures in large-N QCD, hep-th/0404187

62. J. A. Gracey, Nucl. Phys. B 634, 192 (2002) hep-ph/0204266.

63. A.B. Zamolodchikov and V.A. Fateev, Yad. Fiz. 32, 581 (1980);

N.Y. Reshetikhin, Theor. Math. Phys. 63 (1985) 555;

L.A. Takhtajan, Phys. Lett. A 87, 479 (1982);

H.M. Babujian, Phys. Lett. A 90, 479 (1982).

64. D. Müller, Phys. Rev. D 49, 2525 (1994); Phys. Rev. D 58, 054005 (1998) hep-ph/9704406;

A.V. Belitsky and D. Müller, Phys. Lett. B 417, 129 (1998) hep-ph/9709379;

Nucl. Phys. B 527, 207 (1998) hep-ph/9802411; Nucl. Phys. B 537, 397 (1999) hep-ph/9804379.

65. M.F. Sohnius and P.C. West, Phys. Lett. B 100, 245 (1981).

66. P.S. Howe, K.S. Stelle and P.K. Townsend, Nucl. Phys. B 236, 125 (1984).

67. S.J. Gates, M.T. Grisaru, M. Rocek and W. Siegel, Front. Phys. 58, 1 (1983); M.F. Sohnius, Phys. Rept. 128, 39 (1985).

68. D.M. Capper and D.R.T. Jones, Phys. Rev. D 31, 3295 (1985).

69. L. Brink and A. Tollstén, Nucl. Phys. B 249, 244 (1985).

70. A. Smith, Nucl. Phys. B 261, 285 (1985).

71. S.E. Derkachov, D. Karakhanyan and R. Kirschner, Nucl. Phys. B 618, 589 (2001) nlin.si/0102024; Nucl. Phys. B 583, 691 (2000) nlin.si/0003029.

72. M. Scheunert, W. Nahm and V. Rittenberg, J. Math. Phys. 18, 146 (1977); P.D. Jarvis and H.S. Green, J. Math. Phys. 20, 2115 (1979);

L. Frappat, P. Sorba and A. Sciarrino, Dictionary on Lie superalgebras, hep-th/9607161

73. N. Beisert, J.A. Minahan, M. Staudacher and K. Zarembo, JHEP 0309, 010 (2003) hep-th/0306139.

74. N. Beisert, M. Bianchi, J.F. Morales and H. Samtleben, JHEP 0402, 001 (2004) hep-th/0310292.

75. M. Lubcke and K. Zarembo, JHEP 0405, 049 (2004) hep-th/0405055.

76. L. Freyhult, JHEP 0406, 010 (2004) hep-th/0405167.

77. N. Beisert, JHEP 0309, 062 (2003) hep-th/0308074); Nucl. Phys. B 682, 487 (2004) hep-th/0310252.

78. A.V. Ryzhov and A.A. Tseytlin, Towards the exact dilatation operator of $N=4$ super Yang-Mills theory, hep-th/0404215

79. J.A. Minahan, Higher loops beyond the SU(2) sector, hep-th/0405243 
80. A.V. Kotikov and L.N. Lipatov, Nucl. Phys. B 661, 19 (2003); (E) Nucl. Phys. B 685, 405 (2004) hep-ph/0208220;

A.V. Kotikov, L.N. Lipatov and V.N. Velizhanin, Phys. Lett. B 557, 114 (2003) hep-ph/0301021;

A.V. Kotikov, L.N. Lipatov, A.I. Onishchenko and V.N. Velizhanin, hep-th/0404092

81. R. Roiban, On spin chains and field theories, hep-th/0312218

82. D. Berenstein and S.A. Cherkis, Deformations of $N=4 S Y M$ and integrable spin chain models, hep-th/0405215

83. X.J. Wang and Y.S. Wu, Nucl. Phys. B 683, 363 (2004) hep-th/0311073;

B. Chen, X.J. Wang and Y.S. Wu, Phys. Lett. B 591, 170 (2004) hep-th/0403004; JHEP 0402, 029 (2004) hep-th/0401016.

84. O. DeWolfe and N. Mann, JHEP 0404, 035 (2004) hep-th/0401041.

85. P. Di Vecchia and A. Tanzini, $N=2$ super Yang-Mills and the XXZ spin chain, hep-th/0405262

86. L.N. Lipatov, Sov. Phys. JETP 63, 904 (1986)

87. L.N. Lipatov, Phys. Lett. B 251, 284 (1990); Phys. Lett. B 309, 394 (1993).

88. J. Bartels, Nucl. Phys. B 175, 365 (1980).

89. H. Cheng, J.A. Dickinson, C.Y. Lo and K. Olaussen, Phys. Rev. D 23, 534 (1981).

90. J. Kwiecinski and M. Praszalowicz, Phys. Lett. B 94, 413 (1980).

91. R.A. Janik and J. Wosiek, Phys. Rev. Lett. 79, 2935 (1997) hep-th/9610208; Phys. Rev. Lett. 82, 1092 (1999) hep-th/9802100.

92. S.E. Derkachov, G.P. Korchemsky and A.N. Manashov, Nucl. Phys. B 617, 375 (2001) hep-th/0107193.

93. R.J. Baxter, Exactly Solved Models in Statistical Mechanics, (Academic Press, London, 1982).

94. J. Bartels, L. N. Lipatov and G. P. Vacca, Phys. Lett. B 477, 178 (2000) hep-ph/9912423.

95. H. J. De Vega and L. N. Lipatov, Phys. Rev. D 64, 114019 (2001) hep-ph/0107225; Phys. Rev. D 66, 074013 (2002) hep-ph/0204245.

96. S.E. Derkachov, G.P. Korchemsky and A.N. Manashov, Nucl. Phys. B 661, 533 (2003) hep-th/0212169.

97. G.P. Korchemsky and I.M. Krichever, Nucl. Phys. B 505, 387 (1997) hep-th/9704079.

98. S.P. Novikov, S.V. Manakov, L.P. Pitaevskii and V.E. Zakharov, Theory of Solitons: The Inverse Scattering Method, (Consultants Bureau, New York, 1984);

B.A. Dubrovin, I.M. Krichever and S.P. Novikov, Integrable systems - I, Sovremennye problemy matematiki (VINITI), Dynamical systems - 4, 179 (1985);

B.A. Dubrovin, V.B. Matveev and S.P. Novikov, Russ. Math. Surv. 31, 59 (1976).

99. I.M. Krichever, Russ. Math. Surv. 32, 185 (1977); Func. Anal. Appl. 14, 531 (1980); 11, 12 (1977).

100. V. Pasquier and M. Gaudin, J. Phys. A: Math. Gen. 25, 5243 (1992).

101. B.A. Dubrovin, Russ. Math. Surv. 36, 11 (1981).

102. N. Seiberg and E. Witten, Nucl. Phys. B 431, 484 (1994) hep-th/9408099.

103. A. Bilal, Duality in N=2 SUSY SU(2) Yang-Mills Theory: A pedagogical introduction to the work of Seiberg and Witten, hep-th/9601007

104. W. Lerche, Nucl. Phys. Proc. Suppl. B 55, 83 (1997); Fortsch. Phys. 45, 293 (1997) hep-th/9611190.

105. A. Gorsky and A. Mironov, Integrable many-body systems and gauge theories, hep-th/0011197 
106. E. D'Hoker and D.H. Phong, Seiberg-Witten theory and integrable systems, hep-th/9903068

107. A. Marshakov, Seiberg-Witten theory and Integrable Systems, (World Scientific, 1999).

108. A. Gorsky, A. Marshakov, A. Mironov and A. Morozov, Phys. Lett. B 380, 75 (1996) hep-th/9603140.

109. A. Gorsky, S. Gukov and A. Mironov, Nucl. Phys. B 517, 409 (1998) hep-th/9707120.

110. N.A. Nekrasov, Adv. Theor. Math. Phys. 7, 831 (2004) hep-th/0206161.

111. A. Gorsky, I.M. Krichever, A. Marshakov, A. Mironov and A. Morozov, Phys. Lett. B 355, 466 (1995) hep-th/9505035.

112. M. Matone, Phys. Lett. B 357, 342 (1995) hep-th/9506102.

113. P.C. Argyres, M.R. Plesser and A.D. Shapere, Phys. Rev. Lett. 75, 1699 (1995) hep-th/9505100.

114. A. Hanany and Y. Oz, Nucl. Phys. B 452, 283 (1995) hep-th/9505075.

115. J.A. Minahan, Nucl. Phys. B 537, 243 (1999) hep-th/9806246|.

116. P.C. Argyres and S. Pelland, JHEP 0003, 014 (2000) hep-th/9911255.

117. N. Dorey, V.V. Khoze and M.P. Mattis, Nucl. Phys. B 492, 607 (1997) hep-th/9611016.

118. N. Dorey, JHEP 9907, 021 (1999) hep-th/9906011.

119. R. Boels, J. de Boer, R. Duivenvoorden and J. Wijnhout, JHEP 0403, 009 (2004) hep-th/0304061; JHEP 0403, 010 (2004) hep-th/0305189.

120. T.J. Hollowood, JHEP 0310, 051 (2003) hep-th/0305023;

T.J. Hollowood and K. Ohta, hep-th/0405051

121. O. Aharony, S. S. Gubser, J. M. Maldacena, H. Ooguri and Y. Oz, Phys. Rept. 323, 183 (2000) hep-th/9905111.

122. J.M. Maldacena, Phys. Rev. Lett. 80, 4859 (1998) hep-th/9803002;

S.J. Rey, J. Yee, Eur. Phys. J. C 22, 379 (1998) hep-th/9803001.

123. J.K. Erickson, G.W. Semenoff and K. Zarembo, Nucl. Phys. B 582, 155 (2000) hep-th/0003055.

124. R.R. Metsaev, Nucl. Phys. B 625 70, (2002) hep-th/0112044;

R. R. Metsaev and A. A. Tseytlin, Phys. Rev. D 65, 126004 (2002) hep-th/0202109.

125. N. Beisert, C. Kristjansen, J. Plefka, G.W. Semenoff and M. Staudacher, Nucl. Phys. B 650, 125 (2003) hep-th/0208178.

126. D.J. Gross, A. Mikhailov and R. Roiban, Ann. Phys. 301 31, (2002) hep-th/0205066.

127. R. Hernandez and E. Lopez, JHEP 0404, 052 (2004) hep-th/0403139.

128. B.J. Stefanski and A.A. Tseytlin, JHEP 0405, 042 (2004) hep-th/0404133.

129. M. Kruczenski, A.V. Ryzhov and A.A. Tseytlin, Large spin limit of $A d S_{5} \times S^{5}$ string theory and low energy expansion of ferromagnetic spin chains, hep-th/0403120

130. M. Kruczenski and A.A. Tseytlin, Semiclassical relativistic strings in $S^{5}$ and long coherent operators in $\mathcal{N}=4 S Y M$ theory, hep-th/0406189

131. S. Frolov and A.A. Tseytlin, JHEP 0206 007, (2002) hep-th/0204226; Nucl. Phys. B 668 77, (2003) hep-th/0304255; JHEP 0307 016, (2003) hep-th/0306130; Phys. Lett. B 570, 96 (2003) hep-th/0306143.

132. G. Arutyunov, J. Russo and A.A. Tseytlin, Phys. Rev. D 69, 086009 (2004) hep-th/0311004.

133. G. Arutyunov and M. Staudacher, Two-loop commuting charges and the string/gauge duality, hep-th/0403077

134. G. Arutyunov and M. Staudacher, JHEP 0403 004, (2004) hep-th/0310182. 
135. J.A. Minahan, Nucl. Phys. B 648 (2003) 203 hep-th/0209047.

136. B.J. Stefanski, JHEP 0403, 057 (2004) hep-th/0312091].

137. J. Engquist, J.A. Minahan and K. Zarembo, JHEP 0311, 063 (2003) hep-th/0310188.

138. J. Engquist, JHEP 0404, 002 (2004) hep-th/0402092.

139. C. Kristjansen, Phys. Lett. B 586, 106 (2004) hep-th/0402033;

C. Kristjansen and T. Mansson, The circular, elliptic three-spin string from the $S U(3)$ spin chain, hep-th/0406176

140. A.A. Tseytlin, Spinning strings and AdS/CFT duality, hep-th/0311139

141. A. Veselov, Func. Anal. Appl. 22, 1 (1988); J. Moser and A. Veselov, Comm. Math. Phys. 139 , 217 (1991).

142. A. Gorsky, Spin chains and gauge / string duality, hep-th/0308182

143. A.V. Belitsky, A.S. Gorsky and G.P. Korchemsky, Nucl. Phys. B 667, 3 (2003) hep-th/0304028.

144. G. P. Korchemsky, Mod. Phys. Lett. A 4, 1257 (1989).

145. G.P. Korchemsky and G. Marchesini, Nucl. Phys. B 406225 (1993).

146. V.A. Kazakov, A. Marshakov, J.A. Minahan and K. Zarembo, JHEP 0405, 024 (2004) hep-th/0402207.

147. I.M. Krichever, Func. Anal. Appl. 28, 26 (1994).

148. D. Serban and M. Staudacher, JHEP 0406, 001 (2004) hep-th/0401057.

149. N. Beisert, V. Dippel and M. Staudacher, A novel long range spin chain and planar $N=4$ super Yang-Mills, hep-th/0405001

G. Arutyunov, S. Frolov and M. Staudacher, Bethe ansatz for quantum strings, hep-th/0406256

150. C.G. Callan, H.K. Lee, T. McLoughlin, J.H. Schwarz, I. Swanson and X. Wu, Nucl. Phys. B 673, 3 (2003) hep-th/0307032;

C.G. Callan, T. McLoughlin and I. Swanson, Higher impurity AdS/CFT correspondence in the near-BMN limit, hep-th/0405153

I. Swanson, On the integrability of string theory in $A d S_{5} \times S^{5}$, hep-th/0405172

151. A.M. Polyakov, Nucl. Phys. B 164171 (1980).

152. S. Moch, J. A. M. Vermaseren and A. Vogt, Nucl. Phys. B 688, 101 (2004) hep-ph/0403192.

153. M. Kruczenski, JHEP 0212, 024 (2002) hep-th/0210115.

154. Y.M. Makeenko, JHEP 0301, 007 (2003) hep-th/0210256. 